\title{
Education and training for preventing and minimizing workplace aggression directed toward healthcare workers (Review)
}

Geoffrion S, Hills DJ, Ross HM, Pich J, Hill AT, Dalsbø TK, Riahi S, Martínez-Jarreta B, Guay S

Geoffrion S, Hills DJ, Ross HM, Pich J, Hill AT, Dalsbø TK, Riahi S, Martínez-Jarreta B, Guay S.

Education and training for preventing and minimizing workplace aggression directed toward healthcare workers.

Cochrane Database of Systematic Reviews 2020, Issue 9. Art. No.: CD011860.

DOI: 10.1002/14651858.CD011860.pub2.

www.cochranelibrary.com 
TABLE OF CONTENTS

HEADER 1

ABSTRACT

PLAIN LANGUAGE SUMMARY

SUMMARY OF FINDINGS

BACKGROUND

OBJECTIVES

METHODS

RESULTS

Figure 1.

Figure 2.

Figure 3.

DISCUSSION

AUTHORS' CONCLUSIONS

ACKNOWLEDGEMENTS

REFERENCES

CHARACTERISTICS OF STUDIES

DATA AND ANALYSES

Analysis 1.1. Comparison 1: Number of episodes of aggression, Outcome 1: CRCT short-term follow-up

Analysis 1.2. Comparison 1: Number of episodes of aggression, Outcome 2: CBA short-term follow-up

Analysis 1.3. Comparison 1: Number of episodes of aggression, Outcome 3: CBA short-term follow-up

Analysis 1.4. Comparison 1: Number of episodes of aggression, Outcome 4: CRCT long-term follow-up

Analysis 2.1. Comparison 2: Personal knowledge about aggression, Outcome 1: Knowledge about aggression (RCT/CRCT)— short-term follow-up

Analysis 2.2. Comparison 2: Personal knowledge about aggression, Outcome 2: Knowledge about aggression (RCT/CRCT)— long-term follow-up

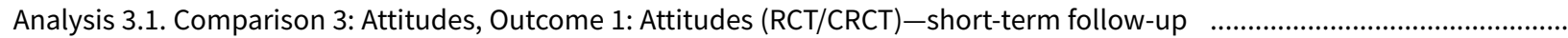

Analysis 4.1. Comparison 4: Skills, Outcome 1: Skills (RCT/CRCT)-short-term follow-up

Analysis 5.1. Comparison 5: Adverse personal outcomes, Outcome 1: Adverse personal (RCT/CRCT)-short-term follow-up .... ADDITIONAL TABLES

APPENDICES

HISTORY

CONTRIBUTIONS OF AUTHORS

DECLARATIONS OF INTEREST

SOURCES OF SUPPORT

DIFFERENCES BETWEEN PROTOCOL AND REVIEW

INDEX TERMS 
[Intervention Review]

\section{Education and training for preventing and minimizing workplace aggression directed toward healthcare workers}

Steve Geoffrion ${ }^{1}$, Danny J Hills², Heather M Ross³ ${ }^{3}$ Jacqueline Pich4, April T Hill5, Therese K Dalsbø ${ }^{6}$, Sanaz Riahi7,8, Begoña MartínezJarreta ${ }^{9}$, Stéphane Guay 10

1École de psychoéducation, Université de Montreal, Montreal, Canada. 2School of Health, Federation University, Ballarat, Australia. ${ }^{3}$ Edson College of Nursing and Health Innovation, Arizona State University, Phoenix, AZ, USA. ${ }^{4}$ Faculty of Health, University of Technology Sydney, Ultimo, Australia. ${ }^{5}$ College of Nursing and Health Innovation, Arizona State University, Phoenix, AZ, USA. ${ }^{6}$ Norwegian Institute of Public Health, Oslo, Norway. ${ }^{7}$ Ontario Shores Centre for Mental Health Sciences, Whitby, Canada. ${ }^{8}$ Department of Psychiatry, University of Toronto, Toronto, Canada. ${ }^{9}$ Faculty of Medicine, University of Zaragoza, Zaragoza, Spain. ${ }^{10}$ School of Criminology, University of Montreal, Montreal, Canada

Contact address: Steve Geoffrion, s.geoffrion@umontreal.ca.

Editorial group: Cochrane Work Group.

Publication status and date: New, published in Issue 9, 2020.

Citation: Geoffrion S, Hills DJ, Ross HM, Pich J, Hill AT, Dalsbø TK, Riahi S, Martínez-Jarreta B, Guay S. Education and training for preventing and minimizing workplace aggression directed toward healthcare workers. Cochrane Database of Systematic Reviews 2020, Issue 9. Art. No.: CD011860. DOI: 10.1002/14651858.CD011860.pub2.

Copyright @ 2020 The Cochrane Collaboration. Published by John Wiley \& Sons, Ltd.

\section{A B S T R A C T}

\section{Background}

Workplace aggression constitutes a serious issue for healthcare workers and organizations. Aggression is tied to physical and mental health issues at an individual level, as well as to absenteeism, decreased productivity or quality of work, and high employee turnover rates at an organizational level. To counteract these negative impacts, organizations have used a variety of interventions, including education and training, to provide workers with the knowledge and skills needed to prevent aggression.

\section{Objectives}

To assess the effectiveness of education and training interventions that aim to prevent and minimize workplace aggression directed toward healthcare workers by patients and patient advocates.

\section{Search methods}

CENTRAL, MEDLINE, Embase, six other databases and five trial registers were searched from their inception to June 2020 together with reference checking, citation searching and contact with study authors to identify additional studies.

\section{Selection criteria}

Randomized controlled trials (RCTs), cluster-randomized controlled trials (CRCTs), and controlled before and after studies (CBAs) that investigated the effectiveness of education and training interventions targeting aggression prevention for healthcare workers.

\section{Data collection and analysis}

Four review authors evaluated and selected the studies resulting from the search. We used standard methodological procedures expected by Cochrane. We assessed the certainty of evidence using the GRADE approach. 


\section{Main results}

We included nine studies-four CRCTs, three RCTs, and two CBAs-with a total of 1688 participants. Five studies reported episodes of aggression, and six studies reported secondary outcomes. Seven studies were conducted among nurses or nurse aides, and two studies among healthcare workers in general. Three studies took place in long-term care, two in the psychiatric ward, and four in hospitals or health centers. Studies were reported from the United States, Switzerland, the United Kingdom, Taiwan, and Sweden.

All included studies reported on education combined with training interventions. Four studies evaluated online programs, and five evaluated face-to-face programs. Five studies were of long duration (up to 52 weeks), and four studies were of short duration. Eight studies had short-term follow-up (<3 months), and one study long-term follow-up ( $>1$ year). Seven studies were rated as being at "high" risk of bias in multiple domains, and all had "unclear" risk of bias in a single domain or in multiple domains.

\section{Effects on aggression}

\section{Short-term follow-up}

The evidence is very uncertain about effects of education and training on aggression at short-term follow-up compared to no intervention (standardized mean difference [SMD] $-0.33,95 \%$ confidence interval $[\mathrm{Cl}]-1.27$ to $0.61,2 \mathrm{CRCTs}$; risk ratio $[\mathrm{RR}] 2.30,95 \% \mathrm{Cl} 0.97$ to $5.42,1$ CBA; SMD -1.24, 95\% Cl -2.16 to -0.33, 1 CBA; very low-certainty evidence).

\section{Long-term follow-up}

Education may not reduce aggression compared to no intervention in the long term (RR $1.14,95 \% \mathrm{Cl} 0.95$ to $1.37,1 \mathrm{CRCT}$; low-certainty evidence).

\section{Effects on knowledge, attitudes, skills, and adverse outcomes}

Education may increase personal knowledge about workplace aggression at short-term follow-up (SMD $0.86,95 \% \mathrm{Cl} 0.34$ to $1.38,1 \mathrm{RCT}$; low-certainty evidence). The evidence is very uncertain about effects of education on personal knowledge in the long term (RR $1.26,95 \%$ $\mathrm{Cl} 0.90$ to 1.75, 1 RCT; very low-certainty evidence). Education may improve attitudes among healthcare workers at short-term follow-up, but the evidence is very uncertain (SMD $0.59,95 \% \mathrm{Cl} 0.24$ to $0.94,2$ CRCTs and 3 RCTs; very low-certainty evidence). The type and duration of interventions resulted in different sizes of effects. Education may not have an effect on skills related to workplace aggression (SMD 0.21 , $95 \% \mathrm{Cl}-0.07$ to $0.49,1 \mathrm{RCT}$ and $1 \mathrm{CRCT}$; very low-certainty evidence) nor on adverse personal outcomes, but the evidence is very uncertain (SMD - $0.31,95 \% \mathrm{Cl}-1.02$ to $0.40,1 \mathrm{RCT}$; very low-certainty evidence).

Measurements of these concepts showed high heterogeneity.

\section{Authors' conclusions}

Education combined with training may not have an effect on workplace aggression directed toward healthcare workers, even though education and training may increase personal knowledge and positive attitudes. Better quality studies that focus on specific settings of healthcare work where exposure to patient aggression is high are needed. Moreover, as most studies have assessed episodes of aggression toward nurses, future studies should include other types of healthcare workers who are also victims of aggression in the same settings, such as orderlies (healthcare assistants). Studies should especially use reports of aggression at an institutional level and should rely on multi-source data while relying on validated measures. Studies should also include days lost to sick leave and employee turnover and should measure outcomes at one-year follow-up. Studies should specify the duration and type of delivery of education and should use an active comparison to prevent raising awareness and reporting in the intervention group only.

\section{PLAIN LANGUAGE SUMMARY}

\section{Do education and training programs reduce aggressive behavior toward healthcare workers?}

\section{What is aggressive behavior?}

The International Labour Organization uses the term "workplace violence" defined as "any action, incident or behaviour that departures from reasonable conduct in which a person is threatened, harmed, injured in the course of, or as a direct result of, his or her work". Experiencing aggressive behavior at work can affect people's ability to do their job well, can cause physical and mental health problems, and can also affect home life. Aggressive behavior may lead to absences from work; some people might leave their job if they experience aggressive behavior.

\section{Why we did this Cochrane Review}

Aggressive behavior exhibited by patients and their families, friends, and carers is a serious problem for healthcare workers. It may affect the quality and safety of the care that healthcare workers can provide. 
Education and training programs have been developed to try to reduce-or eliminate-aggressive behavior at work. These programs are intended to teach and train healthcare workers about:

- their organization's policies and procedures;

- how to assess risks; and

- strategies to control or reduce the chances-and effects-of experiencing aggressive behavior.

\section{What did we do?}

We searched for studies that investigated how well education and training programs prevented or reduced aggression toward healthcare workers.

We included randomized controlled studies, in which the programs that people received were decided at random and studies in which effects of a program were measured before and after among people who completed the program and in another group of people who did not take part.

We wanted to know if education and training programs could:

- reduce the number of incidents of aggressive behavior in healthcare workplaces;

- improve healthcare workers' knowledge, skills, and attitudes toward aggressive behavior; and

- reduce any personal adverse (unwanted or negative) effects noted among healthcare workers who experienced aggressive behavior.

Search date: we included evidence published up to June 2020.

\section{What we found}

We found nine studies including 1688 healthcare workers (including healthcare support staff, such as receptionists) who worked with patients and patients' families, friends, and carers. These studies compared the effects of receiving an education and training program to the effects of not receiving such a program.

Studies were conducted in hospitals or healthcare centers (four studies), in psychiatric wards (two studies), and in long-term care centers (three studies) in the United States, Switzerland, the United Kingdom, Sweden, and Taiwan.

All programs combined education with training provided either online (four studies) or face-to-face (five studies). In eight studies, the people taking part were followed for up to three months (short-term), and in one study for over one year (long-term).

\section{What are the results of our review?}

Education and training programs did not reduce the number of reports of aggressive behavior toward healthcare workers (five studies), possibly because these programs made healthcare workers more likely to report these incidents.

An education and training program might improve healthcare workers' knowledge of aggressive behavior in the workplace in the short term (one study), but we are uncertain whether this would be a long-term effect (one study).

Education programs might improve healthcare workers' attitudes toward aggressive behavior in the short term (five studies), although these results varied depending on the type and length of the program provided.

Education programs might not affect healthcare workers' skills in dealing with aggressive behavior (two studies) and might not affect whether unwanted or negative personal effects were noted after healthcare workers experienced aggressive behavior (one study).

\section{How reliable are these results?}

We are not confident in the results of our review because these results were reported from a small number of studies-some with small numbers of participants-and because some studies showed large differences in results. We identified problems involving the ways some studies were designed, conducted, and reported. Our results are likely to change if further evidence should become available.

\section{Key message}

Although an education and training program might increase healthcare workers' knowledge and positive attitudes, such a program might not affect the number of incidents of aggressive behavior that healthcare workers experience.

More studies are needed, particularly in healthcare workplaces with high rates of aggressive behavior. 


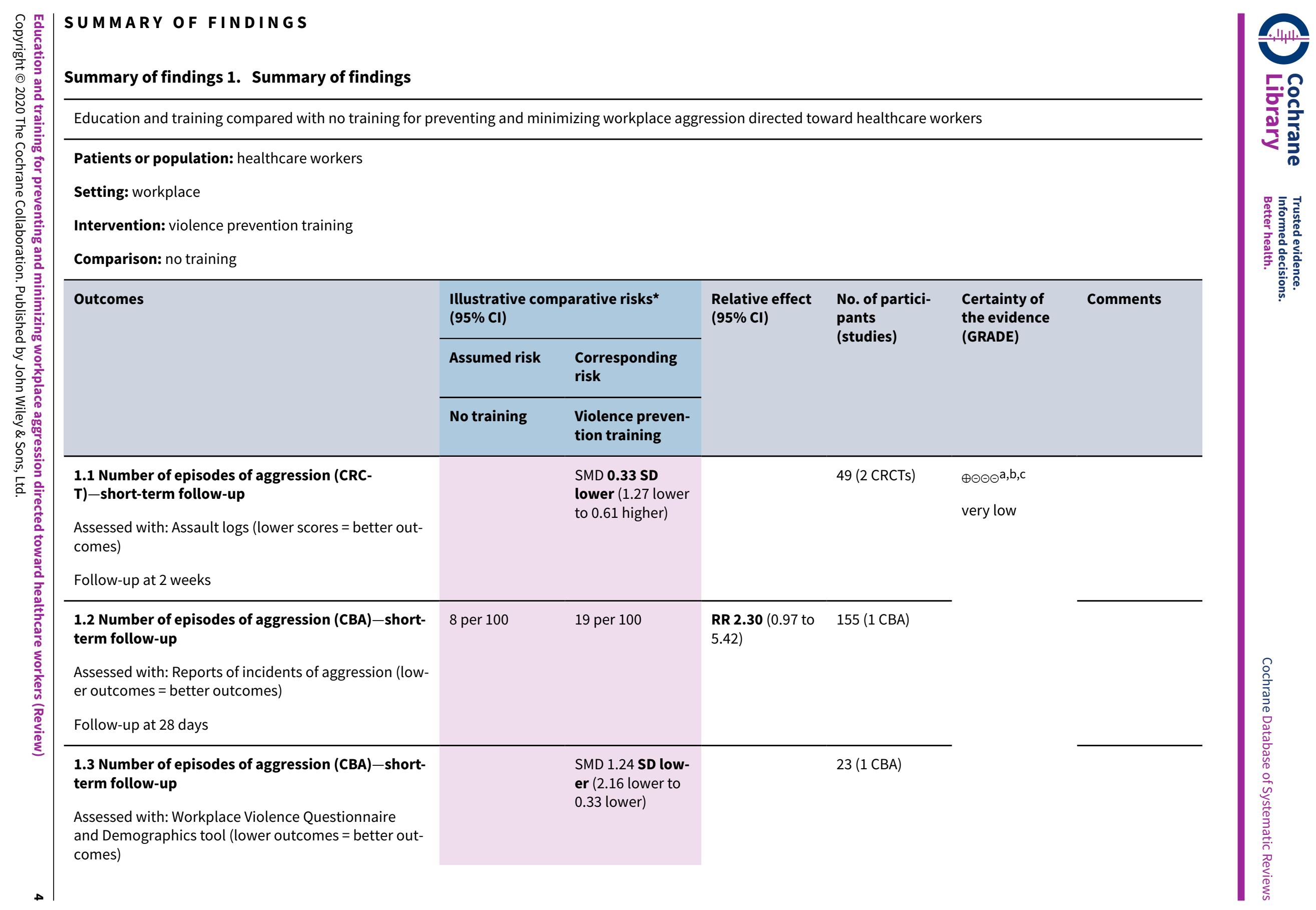




\begin{tabular}{|c|c|c|c|c|c|c|}
\hline \multirow{2}{*}{\multicolumn{2}{|c|}{ 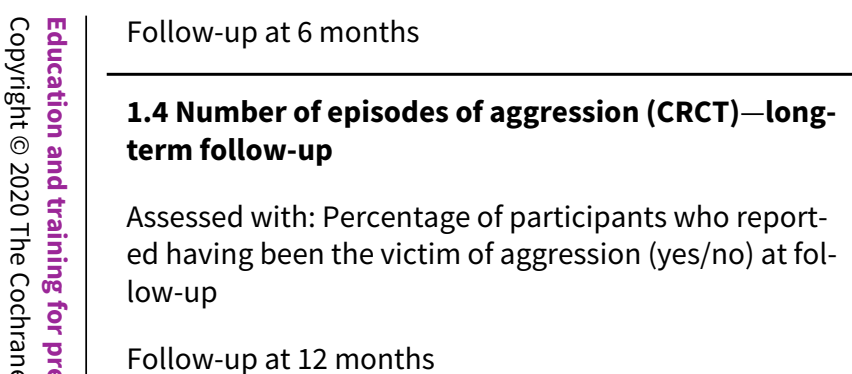 }} & & & & \\
\hline & & 58 per 100 & $\begin{array}{l}66 \text { per } 100 \text { (54 to } \\
76)\end{array}$ & $\begin{array}{l}\text { RR } 1.14 \text { (0.95 to } \\
1.37)\end{array}$ & $\begin{array}{l}291 \\
(1 \text { CRCT })\end{array}$ & $\begin{array}{l}\oplus \oplus \ominus \ominus^{d} \\
\text { low }\end{array}$ \\
\hline 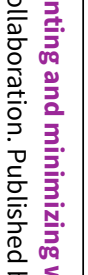 & $\begin{array}{l}\text { 2.1 Knowledge about aggression (RCT/CRC- } \\
\text { T)-short-term follow-up } \\
\text { Assessed with: Knowledge test (higher outcomes = } \\
\text { better outcomes) } \\
\text { Follow-up at } 1 \text { day to } 8 \text { weeks }\end{array}$ & & $\begin{array}{l}\text { SMD } 0.86 \text { SD } \\
\text { higher ( } 0.34 \text { high- } \\
\text { er to } 1.38 \text { higher) }\end{array}$ & & $\begin{array}{l}62 \\
(1 \mathrm{RCT})\end{array}$ & $\begin{array}{l}\oplus \oplus \Theta \Theta b, f \\
\text { low }\end{array}$ \\
\hline 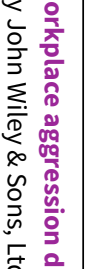 & $\begin{array}{l}\text { 2.2 Knowledge about aggression (RCT/CRCT)-long- } \\
\text { term follow-up } \\
\text { Assessed with: Questions regarding self-perceived im- } \\
\text { provements in knowledge } \\
\text { Follow-up at } 12 \text { months }\end{array}$ & 63 per 100 & $\begin{array}{l}71 \text { per } 100 \\
\text { (65 to } 77)\end{array}$ & $\begin{array}{l}\mathbf{R R} \\
\mathbf{1 . 2 6}(0.90 \text { to } \\
1.75)\end{array}$ & 291 (1 CRCT) & $\begin{array}{l}\oplus \ominus \ominus \ominus^{b, d, g} \\
\text { very low }\end{array}$ \\
\hline 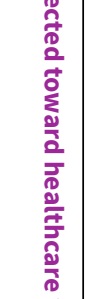 & $\begin{array}{l}\text { 3. Attitudes (RCT/CRCT)-short-term follow-up } \\
\text { Assessed with: Perception of Aggression Scale, Tol- } \\
\text { erance to Aggression Scale, responses to questions } \\
\text { about attitudes toward aggression (higher = better } \\
\text { outcomes) } \\
\text { Follow-up range: } 1 \text { day to } 3 \text { months }\end{array}$ & & $\begin{array}{l}\text { SMD } 0.59 \text { SD } \\
\text { higher ( } 0.24 \text { high- } \\
\text { er to } 0.94 \text { higher) }\end{array}$ & & $\begin{array}{l}683 \text { (2 CRCTs } \\
\text { and } 3 \text { RCTs) }\end{array}$ & $\begin{array}{l}\oplus \ominus \ominus \ominus^{\mathrm{e}, \mathrm{h}} \\
\text { very low }\end{array}$ \\
\hline 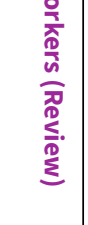 & $\begin{array}{l}\text { 4. Skills (RCT/CRCT)-short-term follow-up } \\
\text { Assessed with: Unspecified questionnaire measuring } \\
\text { empathy (higher score = better outcomes) } \\
\text { Follow-up at } 8 \text { weeks }\end{array}$ & & $\begin{array}{l}\text { SMD } 0.21 \text { SD } \\
\text { higher } \\
\text { (0.07 lower to } \\
0.49 \text { higher) }\end{array}$ & & $\begin{array}{l}198 \\
(1 \mathrm{RCT} \text { and } 1 \\
\text { CRCT })\end{array}$ & $\begin{array}{l}\oplus \Theta \Theta \Theta^{b, j} \\
\text { very low }\end{array}$ \\
\hline & $\begin{array}{l}\text { 5. Adverse personal outcomes (RCT/CRCT)-short- } \\
\text { term follow-up } \\
\text { Assessed with: IMPACS Questionnaire (lower scores = } \\
\text { better outcomes) }\end{array}$ & & $\begin{array}{l}\text { SMD } 0.31 \text { SD } \\
\text { lower } \\
\text { (1.02 lower to } \\
0.40 \text { higher) }\end{array}$ & & 31 (1 RCT) & $\begin{array}{l}\oplus \odot \Theta \Theta^{b, k} \\
\text { very low }\end{array}$ \\
\hline
\end{tabular}


${ }^{*}$ The basis for the assumed risk (e.g. the median control group risk across studies) is provided in footnotes. The corresponding risk (and its $95 \%$ confidence interval) is based on the assumed risk in the comparison group and the relative effect of the intervention (and its $95 \% \mathrm{Cl}$ ).

CBA: controlled before and after study; Cl: confidence interval; $\mathrm{CRCT}$ : cluster-randomized clinical trial; MD: mean difference; RCT: randomized controlled trial; RR: risk ratio; SMD: standardized mean difference.

\section{GRADE Working Group grades of evidence.}

High quality: further research is very unlikely to change our confidence in the estimate of effect.

Moderate quality: further research is likely to have an important impact on our confidence in the estimate of effect and may change the estimate.

Low quality: further research is very likely to have an important impact on our confidence in the estimate of effect and is likely to change the estimate.

Very low quality: we are very uncertain about the estimate.

aDowngraded two levels for high risk of performance and selection bias (Fitzwater 2002). Unclear risk of selection and performance bias (Irvine 2012b).

bDowngraded one level for imprecision due to small sample size and the resulting estimate including little to no effect.

CEvidence from CBA studies was downgraded two levels for high risk of performance and selection bias (Whittington 1996; Anderson 2006), and by one level for imprecision due to the resulting estimate including little to no effect and small sample size.

dDowngraded two levels due to unclear risk of performance and selection bias (Arnetz 2000).

eDowngraded one level for inconsistency and significant heterogeneity.

fowngraded one level due to unclear risk of performance bias (Irvine 2007).

gDowngraded one level due to indirectness.

howngraded two levels due to high risk of attrition bias (Needham 2005), as well as unclear risk of performance bias (Irvine 2007; Irvine 2012a; Irvine 2012b).

Downgraded one level for high risk of performance and selection bias (Fitzwater 2002), as well as unclear risk of performance bias (Irvine 2007; Irvine 2012a; Irvine 2012b).

jowngraded two levels due to unclear risk of selection bias (Irvine 2012a; Irvine 2012b), as well as performance bias (Irvine 2012b).

kDowngraded two levels due to high risk of attrition bias (Needham 2005). 


\section{B A C K G R O U N D}

\section{Aggression in the workplace}

Aggression commonly occurs within the interactional context of work. It is a surprisingly prevalent phenomenon across the globe, with data from the United States, Australia, Japan, Saudi Arabia, and Malaysia indicating that large numbers of working people, in a range of occupations, experience aggression from multiple sources at work (di Martino 2005). The International Labour Organization uses the term "workplace violence" defined as "any action, incident or behaviour that departures from reasonable conduct in which a person is threatened, harmed, injured in the course of, or as a direct result of, his or her work" (ILO 2003). As such, this definition includes different forms of aggression such as physical assault, verbal abuse, bullying, mobbing, and sexual, racial, and psychological harassment. Therefore, in this study, workplace aggression will refer to any episode of aggression toward healthcare workers that resulted in no harm or injury, psychological harm or injury, or physical harm or injury. Workplace aggression may be employed by people external to the workplace (customers/clients and other members of the public) or internal to the workplace (supervisors and other coworkers) to express more immediate distress, frustration, or hostility, or to more deliberately and systematically coerce, intimidate, discriminate, or exert power. Overall, however, aggression from external sources is more prevalent than aggression from coworkers (Cookson 2012; Harrell 2011; LeBlanc 2002; LeBlanc 2006; Packham 2011). We found a large body of evidence related to exposure to workplace aggression from a range of sources and subsequent adverse consequences for individuals and organizations. These include relatively short-lived feelings of distress, fear, and shame; longer-term impact on physical and mental health for individuals (Briggs 2003; Brown 2011; Flannery 2001; Hershcovis 2010; Hills 2014; Hinduja 2007; Hogh 2005a; Hogh 2005b; LeBlanc 2002; Mayhew 2007; Niedhammer 2009; Wieclaw 2006); and impact on their home lives (Lewis 2005). Workplace aggression exposure is also associated with adverse work-related outcomes, including those related to job satisfaction, organizational commitment, and workforce participation intentions (Dupré 2014; Heponiemi 2014; Hills 2014; Lanctôt 2014; Lapierre 2005; LeBlanc 2002). In the healthcare sector, some evidence indicates that exposure of health workers to workplace aggression also impacts the quality and safety of health care provided (Arnetz 2001; Laschinger 2014; Paice 2009; Rosenstein 2008). In their systematic review, Piquero 2013 reported that healthcare workers are among the workers most likely to experience workplace aggression.

\section{Description of the condition}

\section{Aggression in health care}

The process of delivering health care often comprises complex interactions with patients, their advocates, coworkers, and a range of other people peripherally associated or completely unconnected with service delivery (e.g. intruders). It is often stressful work, typically involving working with people who are experiencing distressing conditions or circumstances and suboptimal cognition, affect, or arousal. Consequently, it would be expected that aggression is likely to be an unwelcome feature of healthcare work. Indeed, people working in health care are at high risk of experiencing workplace aggression, second only to people working in protection and security services (Cookson 2012; di Martino 2002;
Estrada 2010; Packham 2011; Parent-Thirion 2007). Furthermore, health workers can be exposed to other occupational conditions associated with higher risk for experiencing workplace aggression, including working alone or in small numbers, working at night, and working in acute care community-based settings (Bulatao 1996; Chappell 2006; Mayhew 2000; Wiskow 2003).

Workplace aggression in health care has become a widely researched phenomenon. This is important because a good deal of evidence suggests that poor reporting practices are the norm rather than the exception in healthcare settings (Farrell 2006; Judy 2009; Mayhew 2001; Parker 2010). Organizational data are dependent on voluntary reporting by staff, yet there is a significant problem with under-reporting of incidents due to lack of clarity about what is a reportable incident, organizational culture, or inadequate support for staff reporting incidents of workplace aggression (Atawneh 2003; Gates 2011; Gerberich 2004; Kvas 2014). Aggression may be viewed by staff and employers as just part of the job, further contributing to under-reporting (Child 2010; VenturaMadangeng 2009). Consequently, survey research may be the most reliable method of estimating the extent of workplace aggression in healthcare settings, despite the likely limitations of recall bias and response bias.

\section{Prevalence of aggression in health care}

A major feature of workplace aggression in healthcare research published since 2000 is that most studies have focused on nurses, with a smaller body of research focused on medical practitioners or mixed populations of health workers, among which nurses are typically the majority of respondents. Most of this research has been exploratory and descriptive in nature, and most studies have estimated 6-month, 12-month, or career prevalence, using cross-sectional, retrospective, self-report survey designs with customized instruments unique to individual studies (Hahn 2008; Hills 2013; Hills 2018; Taylor 2010). Such studyspecific variations render efforts to establish broadly based prevalence rates extraordinarily challenging. Furthermore, the rates of different forms and sources of aggression vary considerably between nations (Camerino 2008; di Martino 2002; Spector 2014). Thus, a systematic review on workplace aggression has estimated that between $7 \%$ and $83 \%$ of healthcare workers have been the target of violent acts (Piquero 2013).

A further complication associated with establishing prevalence rates relates to the imprecision with which workplace aggression is conceptualized and defined in different studies, if explicated at all. Alternative terms include "occupational aggression," "occupational violence," and "counterproductive work behaviour." The terms "aggression" and "violence" are often interchanged. Of greatest concern is the highly problematic use of the term "violence" to include less extreme and non-physical forms of aggression, even though verbal or written expressions of aggression may include highly disturbing threats of violence. Additionally, it has been argued that it is important to distinguish "resistance to care" behavior from aggressive behavior. While appearing similar, the behavioral intentions and the therapeutic responses required are clinically significantly different, with the primarily defensive "resistance to care" frequently exhibited by people with some form of cognitive impairment (Kable 2012). This differentiation appears not to be explicitly considered in much of the health profession workplace aggression literature. 
Despite the challenges of defining and establishing the extent of workplace aggression in health care, patients have been identified as the most common source of aggression, with $10 \%$ to $95 \%$ of respondents reporting that they experienced verbal or physical forms of aggression from patients. Aggression from patients' advocates was reported by $20 \%$ to $50 \%$ of respondents. Studies assessing episodes of aggression typically relied on self-reporting of experiences over the previous 6,12 , or 24 months, suggesting that episodes of aggression are experienced commonly-not rarely-during healthcare practice (Arnetz 2001; Campbell 2011; Carluccio 2010; Farrell 2006; Frank 1998; Gascón 2009; Gerberich 2004; Guay 2014; Hahn 2010; Hegney 2006; Hills 2012; Hills 2013; Hills 2018; Hodgson 2004; Martínez-Jarreta 2007; O'Brien-Pallas 2009; Roche 2010; Spector 2014; Viitasara 2003). When aggression from supervisors and other coworkers has been investigated, it was usually the third most common source, experienced by $3 \%$ to $70 \%$ of survey respondents (Arnetz 2001; Camerino 2008; Campbell 2011; Farrell 2006; Farrell 2010; Hegney 2006; Hills 2012; Hills 2013; Hills 2018; Hodgson 2004; O'Brien-Pallas 2009; Roche 2010).

\section{Prevention and minimization of workplace aggression in health care}

As a consequence of existing evidence on the prevalence of workplace aggression and the wide range of consequences affecting individuals and organizations, there is broad agreement that a diversity of integrated approaches are required to effectively prevent and mitigate aggression and its impact within organizations (ILO 2002; ILO 2003; Mayhew 2000; Mayhew 2004; McCarthy 2004; OSHA 2004; Viitasara 2002). Education and training in the prevention and mitigation of workplace aggression is a key component of any workplace aggression prevention program but can be considered only one of a necessary range of approaches required to address this work health and safety concern. Education and training interventions are unlikely to resolve organizational systems' environmental or cultural challenges. In any case, education and training interventions based on clearly identified needs are lacking (Anderson 2010).

\section{Description of the intervention}

Education and training for prevention and minimization of workplace aggression may comprise any of a broad range of techniques to enhance knowledge and understanding of organizational policies and procedures, legal responsibilities, risk assessment, and control strategies. Further, specific interpersonal skills and behavior management techniques may be tailored to the specific work roles of personnel in the context of the workplace (Chappell 2006; Farrell 2005; ILO 2002; ILO 2003; Mayhew 2000; Mayhew 2001; OSHA 2004).

In this review, education is defined as "the process of imparting knowledge and understanding of organizational policies and procedures, legal responsibilities, and risk assessment and control strategies, including in relation to specific techniques that may be employed in one's work environment, to prevent and mitigate the likelihood and consequences of exposure to workplace aggression." Training is defined as "the process of education about, and rehearsal and simulated or in vivo practice of, cognitive and behavioral skills that may be implemented in one's work to prevent and minimize the likelihood and consequences of exposure to workplace aggression." Thus, healthcare workers should acquire a set of knowledge, attitudes, and skills that aim to prevent aggression in several ways such as de-escalation techniques, effective communication, conflict management, selfdefense, evasion methods, and so on (Spencer 2018).

\section{How the intervention might work}

As highlighted above, education and training interventions, in isolation, are unlikely to resolve systemic, environmental, or cultural challenges that may impact the likelihood and consequences of incidents of workplace aggression in health service organizations. Nonetheless, by improving the knowledge, attitudes, and skills of individuals and groups of healthcare workers related to prevention and minimization of workplace aggression directed toward them by patients and their advocates, it would be expected that the overall number of episodes of aggression, including those resulting in psychological or physical harm or injury, would be reduced. It would also be expected that the number of adverse personal and organizational outcomes attributable to incidents of workplace aggression (e.g. leave days taken, alterations to workforce participation including changing work patterns or attrition, litigation and rehabilitation costs) would be reduced.

\section{Why it is important to do this review}

The capacity to deliver purposeful, safe, and effective responses to potential and escalating aggression is essential for people engaged in any form of human service delivery, including health care, where human interactions are prominent and the risk of aggression may be more prevalent. Unfortunately, there has been a poor history of evaluating education and training programs for aggression minimization and prevention (Beech 2006). Furthermore, available evidence on the impact and outcomes of workplace aggression minimization education and training programs in diverse settings typically shows indeterminate or poor results (Bowers 2006; Gerdtz 2013; Hahn 2013; Heckemann 2015; Hills 2008; Hodgson 2004; Kansagra 2008; Laker 2010; Livingston 2010; Nachreiner 2005; Needham 2005; Price 2015). Nonetheless, clinicians and support personnel recognize its value (Arimatsu 2008; Ceramidas 2010; HEPRU 2003; HEPRU 2008; Judy 2009). It is important to note that the relative absence of evidence for the effectiveness of education and training is no reason to assume that it is ineffective (Richter 2006). Indeed, in the absence of an evidence base, beneficial and possibly life-saving training may be neither sought nor provided (NICE 2006), highlighting the ongoing need for more rigorous evaluation of education and training programs for preventing and minimizing workplace aggression directed toward health workers.

Although reasons for the lack of evidence regarding the protection afforded by education and training are unclear, they may relate in part to necessary plasticity in the application of these techniques for specific situations as they arise. Despite these ongoing concerns, education and training is likely to remain an important component of any structured workplace aggression prevention and minimization program. Precisely what constitutes the key components of effective education and training in workplace aggression prevention and minimization however is unclear.

In this systematic review, we will examine research evidence showing the effects of all types of education and training interventions used by employers in the healthcare sector to build knowledge or skills of healthcare workers as one means of reducing the incidence and adverse outcomes of aggression 
directed toward healthcare workers by patients or their advocates. This review will exclude organizational interventions, application of physical devices, or the introduction of environmental design or re-design features including physical structures. Such structural approaches have been addressed in separate reviews (Spelten 2020; Spencer 2018).

\section{OB JECTIVES}

To assess the effectiveness of education and training interventions that aim to prevent and minimize workplace aggression directed toward healthcare workers by patients and patient advocates.

\section{METHODS}

\section{Criteria for considering studies for this review}

\section{Types of studies}

We considered all published and unpublished randomized controlled trials (RCTs) and controlled before and after studies (CBAs) as eligible for inclusion in this review.

\section{Types of participants}

We included healthcare workers who interact with patients, patient advocates, or both, in any public or private healthcare facility regardless of worker age, gender, or profession. These included:

- physicians and physician assistants;

- dentists;

- nurses and midwives;

- allied health professionals (e.g. physiotherapists, occupational therapists, speech pathologists, pharmacists, respiratory therapists, medical imaging technicians, oral hygienists, podiatrists, dieticians, opticians); and

- healthcare support personnel (e.g. reception staff, healthcare aides or assistants, healthcare security personnel).

\section{Types of interventions}

We included any educational or training intervention undertaken with healthcare workers to improve their knowledge, attitudes, and skills in preventing and minimizing verbal or physical aggression directed toward them and their workplace peers from patients or their advocates. These included interventions designed to enhance knowledge and understanding of legal responsibilities, organizational policies and procedures, and specific risk assessment and control strategies. Interventions included education and training in specific communication and behavior management techniques targeting the diffusion and de-escalation of aggression, violence avoidance and breakaway strategies, and physical restraint of aggressive people.

We included interventions that were mandatory or voluntary; delivered all at once or over multiple sessions; and delivered faceto-face, online, or in blended form and including synchronous or asynchronous components. We included interventions delivered in workplace, educational, and other professional settings. We included stand-alone programs as well as those offered in conjunction with other organizational interventions, but only when such interventions were "controlled for" in the analysis of impact or outcomes, or when they could be determined not to have confounded or biased results of the education and training intervention study.

\section{Types of outcome measures}

Outcome measures included reported clinical events and participant-reported outcomes.

\section{Primary outcomes}

We included studies that evaluated the effects of an education or training intervention among staff in the healthcare sector on the number of episodes of aggression.

\section{Secondary outcomes}

- Personal knowledge about workplace aggression

- Attitudes toward workplace aggression

- Skills related to workplace aggression

- Adverse personal and organizational outcomes attributable to incidents of workplace aggression (e.g. leave days taken, alterations to workforce participation including changing work patterns or attrition, litigation, and rehabilitation costs)

\section{Search methods for identification of studies}

We conducted a systematic search of the literature to identify all published and unpublished RCTs and CBAs that could be considered eligible for inclusion in this review. The literature search identified potentially eligible studies in all languages. If we would have encountered foreign language studies, non-English language papers would have been translated and fully assessed for potential inclusion in the review as necessary.

\section{Electronic searches}

We searched the following electronic databases from their inception to the date of the search specified to identify potential studies.

- Cochrane Central Register of Controlled Trials (CENTRAL), in the Cochrane Library (June 2020).

- MEDLINE (PubMed, June 2020).

- Embase (June 2020).

- Cumulative Index to Nursing and Allied Health Literature (CINAHL) (EBSCO, June 2020).

- PsycINFO (ProQuest, June 2020).

- US National Institute for Occupational Safety and Health bibliographic database of literature on occupational safety and health (NIOSHTIC) (OSH-UPDATE, June 2020).

- NIOSHTIC-2 (OSH-UPDATE, June 2020).

- HSELINE (OSH-UPDATE, June 2020).

- ISDOC (OSH-UPDATE, June 2020).

We used keywords selected from the search strategies supplied in Appendix 1.

\section{Searching other resources}

We also conducted a search of the following.

- ClinicalTrials.gov (www.ClinicalTrials.gov) and the World Health Organization (WHO) trials portal (www.who.int/ictrp/en/).

- WorkSafe Australia. 
- Canadian Centre for Occupational Health and Safety (CCOHS).

- The Campbell Collaboration and social, psychological, educational, and criminological trials register.

We checked the reference lists of all primary studies and review articles for additional references. Finally, we contacted experts in the field to identify additional unpublished materials.

\section{Data collection and analysis}

\section{Selection of studies}

Four review authors (JP, AH, SR, SGe) independently screened titles and abstracts of all potentially eligible studies identified as a result of the search and coded them as "retrieve" (eligible or potentially eligible/unclear) or "do not retrieve." We retrieved the full-text study reports/abstracts/publications, and five review authors (HR, TD, SG, BM-J, SGe) independently screened the full text and identified studies for inclusion. When a study was identified as ineligible for inclusion in the review, we recorded the reason(s) for its exclusion. We resolved disagreements by consensus or by consultation with another person from the review team $(\mathrm{DH}$, $\mathrm{SGe}$. We identified and excluded duplicates and collated multiple reports of the same study, so that each study, rather than each report, is the unit of interest in the review. We recorded this selection process in sufficient detail to complete a PRISMA flow diagram and Characteristics of excluded studies table.

\section{Data extraction and management}

We used a study-specific data collection form for collection of study characteristics, intervention details, and outcome data (Appendix 2). All review authors piloted this form on one study in the review. Four review authors (HR, AH, SG, SGe) extracted study characteristics from the identified included studies.

Using the study-specific data collection form, we extracted the following study characteristics.

- Publication details: authors, email address of corresponding author, date of publication, title, journal name, volume, issue, pages.

- Methods: study design (e.g. RCT/cluster RCT/CBA), including sampling, group allocation and treatment of missing data, study location/s, study setting/s, withdrawals.

- Participants: health worker type/s, total number of participants, number of health worker type sub-populations and proportions (\%), mean age or age range, gender, workplace/s (e.g. mental health, emergency department), work setting/s (e.g. hospital inpatient, hospital outpatient, community), work sector/s (e.g. public, private, non-government), inclusion and exclusion criteria.

- Interventions: description of interventions and cointerventions, targeted knowledge, attitudes and skills, comparison, content of both intervention and control condition, and co-interventions (especially noting if bundled with other organizational interventions), duration, intensity, number commencing, number completing, adherence to protocol.

- Outcomes: description of primary and secondary outcomes specified and collected, measurement instruments used and validation status (e.g. reported/not reported), at which time points reported, controlling for biasing or confounding effects of co-interventions.
- Length of follow-up: time points at which primary and secondary outcomes were collected; categorization to shortterm, medium-term, and long-term follow-up (see further details below in Assessment of heterogeneity).

- Notes: funding for study, notable conflicts of interest of trial authors.

Upon preparation of the final included list of studies, three review authors (DH, SR, SGe) independently extracted data from these study reports. We noted in the Characteristics of included studies table if outcome data were not reported in a usable way. One review author (TD) transferred data into Review Manager 5 (RevMan 5.3.) Another (SGe) made the migration toward Review Manager Web (RevMan Web 2019). We double-checked that data were entered correctly by comparing data presented in the systematic review with information provided in the study reports. Two review authors (JP, SGe) spot-checked study characteristics for accuracy against the study report.

\section{Assessment of risk of bias in included studies}

Four authors of the present review (DH, TD, BM-J, SGe) independently assessed risk of bias for each study using the criteria outlined in the Cochrane Handbook for Systematic Reviews of Interventions (Higgins 2011). Disagreements were resolved by discussion or by consultation with another review author $(\mathrm{AH}, \mathrm{HR}$, JP, SG, or SR). Risk of bias of the included RCTs was assessed according to the following domains.

- Random sequence generation.

- Allocation concealment.

- Blinding of participants and personnel.

- Blinding of outcome assessment.

- Incomplete outcome data.

- Selective outcome reporting.

- Other biases.

Each potential source of bias was graded as high, low, or unclear. Further, a quote from the study report was provided together with a justification for the assessment in the "Risk of bias" table. The risk of bias judgment is summarized across different studies for each of the domains listed. Blinding was considered separately for different key outcomes when necessary (e.g. for unblinded outcome assessment, risk of bias for all-cause mortality may be very different than for a participant-reported pain scale). However, blinding was not found to be necessary for evaluation of risk of bias of the included studies. When information on risk of bias was related to unpublished data or correspondence with a trialist, this was noted in the "Risk of bias" table.

For CBAs, we used a combination of the applicable domains for risk of bias determination for RCTs and elements of the Downs and Black checklist (Downs 1998), as described in Chapter 13 of the Cochrane Handbook for Systematic Reviews of Interventions (Higgins 2011).

\section{Assessment of bias in conducting the systematic review}

We conducted the review according to this published protocol and reported any deviations from it in the Differences between protocol and review section of the systematic review. 


\section{Measures of treatment effect}

Outcome data for each study were entered into the data tables in Review Manager Web to calculate treatment effects (RevMan Web 2019). We used risk ratios (RRs) for dichotomous outcomes and standardized mean differences (SMDs) for continuous outcomes, or other types of data as reported by study authors. When only effect estimates and their 95\% confidence intervals (Cls) or standard errors were reported in studies, we entered these data into Review Manager Web using the generic inverse-variance method. We ensured that higher scores for continuous outcomes have the same meaning for the particular outcome, explained the direction to the reader, and reported when the directions were reversed, if this was necessary. When results could not be entered either way, we described them in the Characteristics of included studies table, or we entered the data into Additional tables.

\section{Unit of analysis issues}

For studies that employed a cluster-randomized controlled trial (CRCT) design and that reported sufficient data for inclusion in the meta-analysis but did not make an allowance for the design effect, we calculated the design effect based on a fairly large assumed intracluster correlation of 0.10 (Appendix 3). We based this assumption of 0.10 as a realistic estimate by analogy to studies about implementation research (Campbell 2001). We followed the methods stated in the Cochrane Handbook for Systematic Reviews of Interventions to perform the calculations (Higgins 2011).

\section{Dealing with missing data}

We contacted investigators or study sponsors to verify key study characteristics and obtain missing numerical outcome data when possible (e.g. when a study is identified as an abstract only). When this was not possible and the missing data were thought to introduce serious bias, we explored the impact of including such studies in the overall assessment of results by conducting a sensitivity analysis.

If numerical outcome data such as standard deviations or correlation coefficients were missing and we could not obtain these from trial authors, we calculated them from other available statistics such as $P$ values, according to the methods described in the Cochrane Handbook for Systematic Reviews of Interventions (Higgins 2011).

\section{Assessment of heterogeneity}

We assessed the homogeneity of the results of all included studies based on similarity of study design, intervention types, outcomes, and follow-up. We considered interventions to be different when they included education only or education combined with training. RCTs and CBAs were considered separately.

We categorized studies based on mode of delivery (online or faceto-face) and on their duration (short for less than a week, long for one week or longer, and self-paced). We did not assume that these differences could cause differences in the effect estimates. Still, we did run subgroup analysis to check for any differences in both mode of delivery and length of interventions. We reported the results of this analysis both combined and separated when subgroup differences were found.

Further, follow-up times were categorized into short-term (six months and less), medium-term (between six months and 12 months), and long-term (12 months and longer) follow-up and were regarded as different.

Statistical heterogeneity was assessed using the $\mathrm{I}^{2}$ statistic (Higgins 2011), based on the following as a rough guide for interpretation: $0 \%$ to $40 \%$ might not be important; $30 \%$ to $60 \%$ may represent moderate heterogeneity; $50 \%$ to $90 \%$ may represent substantial heterogeneity; $75 \%$ to $100 \%$ considerable heterogeneity. In cases of substantial heterogeneity (defined as $I^{2} \geq 50 \%$ ), we explored the data further, including subgroup analyses, in an attempt to explain the heterogeneity.

\section{Assessment of reporting biases}

Because we were not able to pool 10 or more trials in any single meta-analysis, we did not explore possible small-study biases via funnel plot examination.

\section{Data synthesis}

We pooled the data from studies judged to be homogeneous using Review Manager Web software (RevMan Web 2019). If more than one study provided usable data for any single comparison, we performed meta-analysis. When studies were statistically heterogeneous, we used a random-effects model. Otherwise, we used a fixed-effect model. When using the random-effects model, we conducted a sensitivity check by using the fixed-effect model to reveal differences in results. We included a $95 \%$ confidence interval for all estimates.

When multiple trial arms were reported in a single trial, we included only the relevant arms. If two comparisons were combined in the same meta-analysis, we halved the control group to avoid doublecounting.

\section{Subgroup analysis and investigation of heterogeneity}

The original protocol intended to carry out subgroup analyses if a sufficient number of studies were found based on types of delivery and length of intervention. As such, we carried out subgroup analyses when a sufficient number of studies with substantial heterogeneity $\left(I^{2}>50 \%\right)$ were found among the included studies based on:

- types of delivery (face-to-face or online); and

- duration of intervention (short, long, or self-paced).

\section{Sensitivity analysis}

We originally planned to carry out sensitivity analysis to test the robustness of our meta-analysis results by omitting studies that we judged to be at high risk of bias. However, we did not find a sufficient number of studies to perform sensitivity analyses.

\section{Summary of findings and assessment of the certainty of the evidence}

A "Summary of findings" table was created for each of the following outcomes.

- Episodes of aggression.

- Changes in personal knowledge, attitudes, and skills related to workplace aggression.

- Adverse personal and organizational outcomes attributable to incidents of workplace aggression. 
We evaluated the quality of available evidence using the GRADE approach. We generated a "Summary of findings" table that provides outcome-specific information concerning the overall quality of evidence from studies included in the comparison, the magnitude of effect of the interventions examined, and the sum of available data on outcomes considered. We included information on the primary and secondary outcomes of our review. We assessed the quality of evidence using several factors.

- Limitations in study design and implementation of available studies.

- Indirectness of evidence.

- Unexplained heterogeneity or inconsistency of results.

- Imprecision of effect estimates.

- Potential publication bias.

For each outcome, we classified the quality of evidence according to the following categories.

- High quality: further research is very unlikely to change our confidence in the estimate of effect.

- Moderate quality: further research is likely to have an important impact on our confidence in the estimate of effect, and may change the estimate.

- Low quality: further research is very likely to have an important impact on our confidence in the estimate of effect, and is likely to change the estimate.

- Very low quality: we are very uncertain about the estimate.

We used the five GRADE considerations (study limitations, consistency of effect, imprecision, indirectness, and publication bias) to assess the quality of a body of evidence as it relates to the studies that contributed data to meta-analyses for the pre-specified outcomes. We adhered to the methods and recommendations described in Section 8.5 and Chapter 12 of the Cochrane Handbook for Systematic Reviews of Interventions, using GRADEpro software (GRADEPro 2014; Higgins 2011). We justified all decisions to downgrade the quality of RCTs or to upgrade the quality of CBAs using footnotes, and we made comments to aid the reader's understanding of the review when necessary. Criteria for upgrading included a large effect size, a reported dose-response gradient, and the effects of all plausible confounding factors working against the intervention.

\section{Reaching conclusions}

We based our conclusions only on findings from the quantitative or narrative synthesis of studies included in this review. We avoided making recommendations for practice based on more than just the evidence, such as values and available resources. Our implications for research suggest priorities for future research and outline remaining uncertainties in this area.

\section{RE S U L TS}

\section{Description of studies}

See Characteristics of included studies and Excluded studies tables.

\section{Results of the search}

Results of the search strategy are presented in Figure 1. Through the search, we found a total of 4803 references, 37 of which we deemed potentially eligible for inclusion. We then accessed the full-text articles of these studies and, upon closer examination, excluded 28 manuscripts (Table 1). Finally, we decided that nine studies met the inclusion criteria, and we included them in the review (Anderson 2006; Arnetz 2000; Fitzwater 2002; Irvine 2007; Irvine 2012a; Irvine 2012b; Ming 2019; Needham 2005; Whittington 1996). We tried twice to contact the authors of an included study to obtain more information about the data, but without success. We therefore available data provided in the published articles for all included studies. 
Figure 1. Figure 1. Prisma flow diagram of search and screening results.

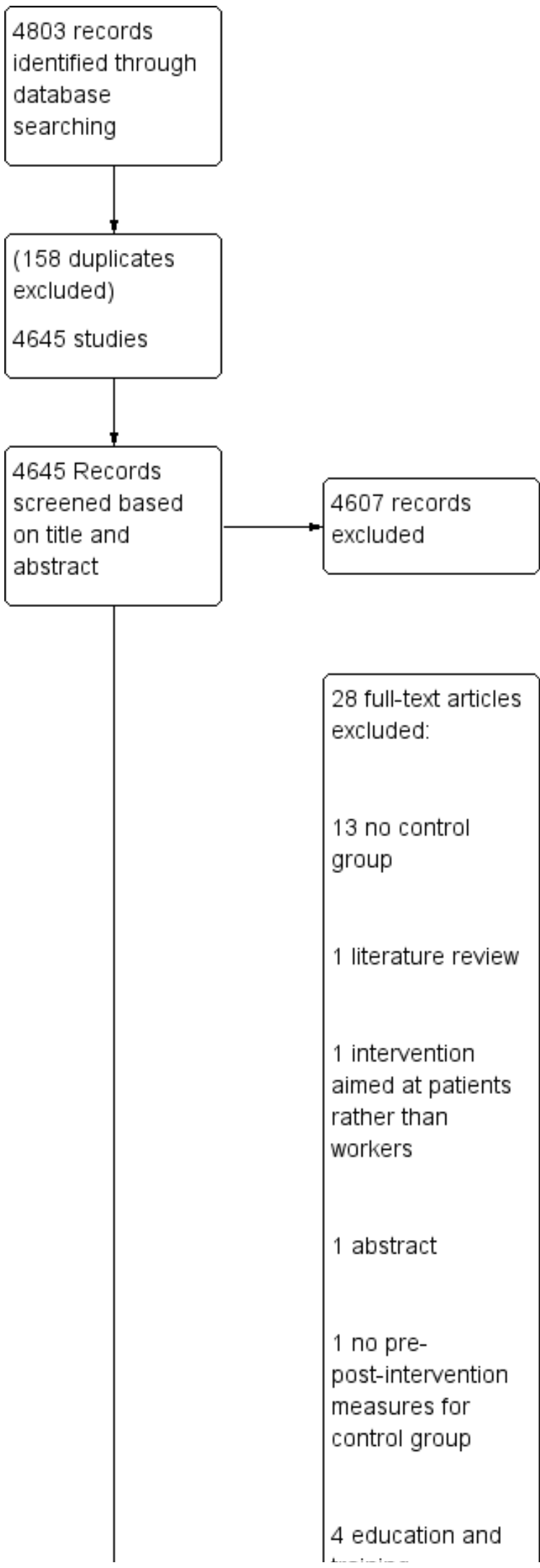


Figure 1. (Continued)

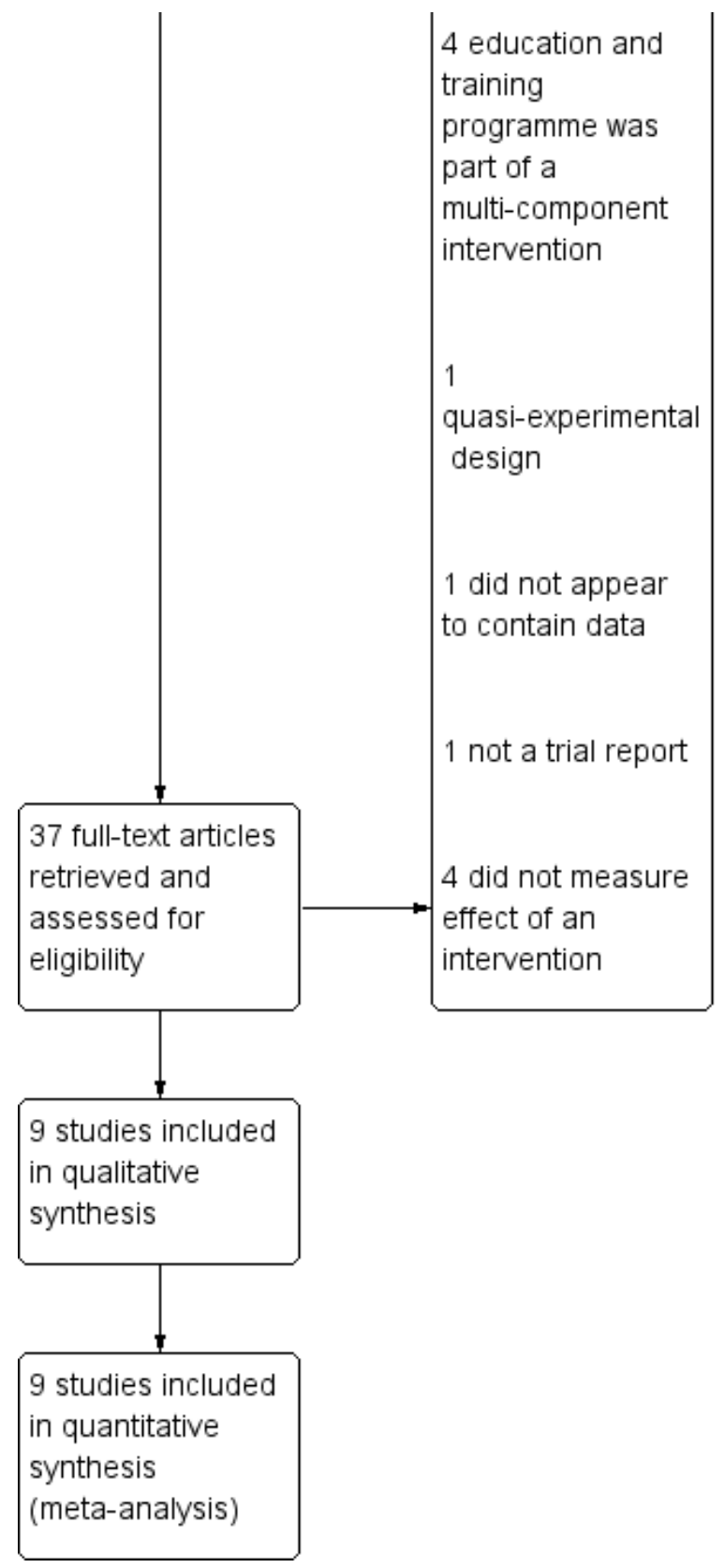

\section{Included studies}

\section{Study design}

Five studies reported on the number of episodes of aggression directed toward healthcare workers: three CRCTs (Arnetz 2000; Fitzwater 2002; Irvine 2012b), and two CBAs (Anderson 2006; Whittington 1996). In addition, three RCTs-Irvine 2007; Irvine 2012a; Ming 2019-and three CRCTs-Arnetz 2000; Irvine 2012b; Needham 2005-reported on secondary outcomes.

\section{Participants}

Two RCTs-Irvine 2007; Irvine 2012a-and one CRCT-Irvine 2012b-were conducted among nurse aides working in long-term care facilities ( $N=62,159$, and 103 , respectively). Participants for
Irvine 2007 and Irvine 2012a were drawn from people in the United States who answered Internet advertising. Participants for Irvine 2012b were drawn from two long-term care facilities in Cincinnati, Ohio, USA. Another CRCT was conducted among 20 certified nurse assistants working at two nursing homes in midwestern United States (Fitzwater 2002).

One CRCT-Needham 2005-and one CBA-Whittington 1996were carried out among psychiatric ward nurses and included a sample size of 58 nurses and 155 nurses, respectively, at followup. Whittington 1996 was conducted in London, United Kingdom. Needham 2005 was conducted in German-speaking portions of Switzerland. 
One CBA-Anderson 2006-investigated effects of a training program among 43 hospital workers in a small community hospital in the United States. One RCT examined effects of simulation training among 392 nurses working at a medical center in Taipei, Taiwan (Ming 2019). Finally, one CRCT-Arnetz 2000-examined the effects of a practical intervention program at 47 healthcare workplaces in Stockholm, Sweden, representing a total of 686 workers at follow-up.

\section{Types of Interventions}

\section{See Table 2.}

Four studies evaluated online programs and five studies evaluated face-to-face programs. All studies provided education combined with training.

\section{Online education programs-short duration}

Participants who received training in Anderson 2006 underwent a three-hour online training program comprising five modules, completed within a 30-day period. The training program covered material such as identifying triggers for violence and exploring why violence happens and provided resources and suggestions for diffusing anger and for debriefing/follow-up after an episode of violence.

\section{Online education programs-long duration}

Two of the included studies-Irvine 2012a and Irvine 2012bconsisted of two online training sessions offered one week apart. Study authors did not specify the length of each training session, although they did mention that the training was based on Irvine 2007, which was self-paced. The first session was dedicated to deescalation skills, and the second taught situation-specific advanced skills (e.g. pulling hair).

\section{Online education programmes-self-paced}

The intervention used by Irvine 2007 consisted of 155 Web pages, 11 video vignettes, 16 narrator video clips, 71 voiceover clips, and 3 video testimonials. These interventions aimed to provide skills on how to approach agitated long-term care residents and how to de-escalate situations, and were to be completed in a one-day selfpaced online training session. Study authors did not specify the length of the training program, which is somewhat variable due to its self-paced nature, but they reported that participants normally completed the program within a single day.

\section{Face-to-face program-short duration}

Fitzwater 2002 provided participants in the intervention group with two assault prevention sessions, each lasting two hours. These training sessions were provided by a master's level psychiatric nurse. The intervention was designed to prevent and reduce violent incidents and involved topics such as reasons for violence, effective communication, signs of impending violence, and how to protect their own as well as residents' safety.

The intervention used in Whittington 1996 was based on a cyclical model of violence in psychiatric units and consisted of two components. The first dealt with prevention of imminent violence, and the second addressed dealing with possible psychological consequences of the assault. Training took place over a seven-hour period in a single day.
Participants in Ming 2019 received a three-hour teaching session, which included an hour-long discussion on the topic of workplace violence, review of case videos, demonstrations of workplace violence prevention, treatment, self-defense and evasion methods, and role-playing of situational simulations.

\section{Face-to-face program-long duration}

The intervention described for Needham 2005 was a training program that consisted of 20 lessons, with each lesson lasting 50 minutes. It was delivered by trained psychiatric nurses over five consecutive days. The lessons covered topics such as causes and types of aggression, conflict management, communication, behavior during aggression, prevention of aggression, and postaggression procedures.

\section{Face-to-face program-extended duration}

The intervention used by Arnetz 2000 consisted of contact between project co-ordinators and workers who registered a violent incident. These incidents were later presented at regular staff meetings and were discussed within the group.

\section{Outcomes}

\section{Primary outcome-episodes of aggression}

Fitzwater 2002 and Irvine 2012b measured the effectiveness of training in reducing aggression using an Assault Log, which was a record-keeping process to document and describe physical assaults by patients. Workers completed this form after each workday. Fitzwater 2002 reported the mean total number of reported events for participants at each site in the two weeks before the intervention and in the two weeks following the intervention. Irvine 2012b reported the mean number of daily occurrences of aggression. In both studies, participants in the control group were compared to participants who received the intervention. For these studies, we used the change between baseline and followup in the mean number of daily occurrences of aggression for the intervention and control groups to determine standard mean differences (SMDs).

Whittington 1996 evaluated the effectiveness of a training intervention among nurses using the number of notified assaults on staff during the 28 days preceding training and the 28 days immediately following training, determined by contacting all available staff in participating wards every day about any notifiable aggression that had occurred over the past 24 to 48 hours. This was used by study authors to compute the percentages of workers in intervention and control groups who had been the target of an aggression at baseline (i.e. in the 28 days before the intervention) and at follow-up (i.e. in the 28 days following the intervention). We used these percentages to estimate the number of events (i.e. the number of staff that had been the target of aggression) and to calculate a risk ratio (RR) for intervention and control groups at follow-up.

Anderson 2006 assessed the effectiveness of a training intervention using the Workplace Violence Questionnaire and Demographics Tool, a self-report questionnaire that documented the frequency and type of workplace violence events. This instrument was developed by the study author based on the Conflict Tactics Scale (Straus 1979), as well as the Wyatt Sex History questionnaire (Wyatt 1995). The questionnaire lists multiple events of aggression. Participants were instructed to indicate which of these events had 
occurred to them over the past six months. This questionnaire documented the frequency and type of events of aggression. For the current analysis, the mean number of events per participant was calculated at baseline and at follow-up for intervention and control groups. The SMD in change in mean number of events between baseline and follow-up (six months) was used to determine the efficacy of the intervention in reducing the frequency of episodes of aggression.

Arnetz 2000 asked participants if they had been the target of aggression over the past year at two times: at baseline-before the intervention-and at follow-up one year later-at the end of the intervention. The percentage of participants who answered "yes" to this question was determined by adding together the percentages of participants who answered "yes, once or twice" with those who answered "yes, several times." This combined percentage was then used to estimate the number of participants who reported having been the victim of aggression at follow-up, at the end of one-year intervention. We then calculated an RR based on these estimates.

\section{Secondary outcomes}

\section{Personal knowledge about workplace aggression}

Irvine 2007 tested participants' personal knowledge about workplace aggression by presenting three video vignettes demonstrating an example of workplace aggression (e.g. a patient in a wheelchair swinging his arms violently). At the end of each vignette, participants were asked a single multiple choice question about what to do in the previously depicted situation. Participants indicated their responses by selecting the option that corresponded to what they thought was the correct response. Participants were tested before intervention and again, at the end of the self-paced intervention. Study authors reported the mean proportions of correct responses both before intervention and at follow-up for intervention and control groups. For the present analyses, we used the SMD in the proportion of correct responses.

Arnetz 2000 assessed the effects of intervention on worker knowledge by asking participants three questions regarding whether the project had given them better knowledge of (1) risk situations for aggression toward staff; (2) how potentially dangerous situations could be avoided or attenuated; and (3) how best to handle a patient or another person who became aggressive toward them in the workplace. We selected the first measure (i.e. risk situations for aggression toward staff) to be included in the analysis as it fitted the definition of personal knowledge about workplace aggression and it better encompassed the general aspect of this outcome. For this measure, we estimated the number of respondents who answered "yes" based on the percentages reported by study authors and the number of participants who answered at 12 months' follow-up. These data were then used to calculate RRs for the present analyses.

\section{Attitudes toward patient aggression}

Irvine 2007 assessed the effects of intervention on worker attitudes toward aggression using a 13-item unspecified scale. Each item evaluated participants' agreement with the importance of certain behavioral responses to aggression and asked participants to indicate their level of agreement on a 7-point Likert scale $(1=$ completely agree; 7 = completely disagree). For each participant, attitude was measured as the mean of response across these 13 items at each testing interval. Assessments were made before intervention and again at follow-up, at the end of the selfpaced intervention. Study authors reported the mean attitude response for cohorts both before intervention and at follow-upone business day after the end of the self-paced intervention. For the present analysis, we used the SMD in change in attitude scores at one-day follow-up to assess the efficacy of the intervention in improving worker attitudes toward aggression.

Irvine 2012a used an unspecified five-item questionnaire to assess effects of intervention on worker attitudes. Respondents were asked to indicated their level of agreement/disagreement using a 7-point Likert scale ( 1 = completely disagree; 7 = completely agree). The score was the mean value across these five items. For the present analysis, we used the SMD in mean score changes between baseline and eight-week follow-up assessments to determine the efficacy of the intervention in improving worker attitudes toward violence.

Irvine 2012b evaluated attitudes toward aggression using a single item. Participants were asked the extent to which they agreed or disagreed with the statement, "I believe that residents act aggressively because they have unmet needs." Participants were asked to indicate their level of agreement/disagreement on a 7-point Likert scale ( $1=$ completely disagree; $7=$ completely agree). Study authors reported the mean values for control and intervention groups before intervention and at eight weeks' followup. For the present analyses, we used the SMD in change in scores between baseline and follow-up scores to evaluate the efficacy of intervention in changing worker attitudes for them to be more understanding of the causes of aggression.

Ming 2019 measured the effects of intervention on worker attitudes using seven items from the Management of Aggression and Violence Attitude Scale (Duxbury 2002; Duxbury 2008). Each of these items contains a statement regarding the causes of aggression (e.g. "It is largely situations that can contribute toward the expression of aggression by patients"). Participants were asked to indicate their level of agreement/disagreement with each item using a 5-point Likert scale ranging from 1 (i.e. "strongly disagree") to 5 (i.e. "strongly agree"). For each participant, individual test item response values were summed to yield a global score from 7 to 35 . Study authors reported the mean global score values for intervention and control groups at baseline and at follow-up three months later. We calculated the SMD in change in scores between intervention and control groups to establish the effectiveness of the intervention in improving attitudes toward patient aggression.

Needham 2005 assessed effects of an intervention on worker attitudes using the short version of the Perception of Aggression Scale and the Tolerance Scale, respectively (Needham 2004; Whittington 2002). Each of the 12 items on the short Perception of Aggression Scale asks participants to indicate their level of agreement with a statement (e.g. "aggression is an emotional outlet") on a 5-point Likert scale ( 1 = strongly disagree; 5 = strongly agree). The Tolerance Scale is derived from the long version of the Perception of Aggression Scale (Jansen 1997). Because this scale stemmed from the Perception of Aggression Scale, we retained only the short version of the Perception of Aggression Scale for this review. Needham 2005 reported mean Perception of Aggression (POAS-S) "positive" scale results for intervention and control groups at baseline and at follow-up 90 days later. Individual scores were calculated by adding the response value of the items on each scale. For the present analyses, we calculated the SMD in change 
in scores between intervention and control groups to establish the effectiveness of the intervention in changing workers' attitudes toward patient aggression.

\section{Skills related to workplace aggression}

Irvine 2012a used four items from the Personal Accomplishment Scale to assess effects of intervention on the empathy of participants toward residents (Ray 1994). Irvine 2012b assessed caregiver empathy with a single item from the Personal Accomplishment Scale (Ray 1994): "even if a resident sometimes is verbally or physically aggressive toward me, I can easily understand how he/she feels about things." In both studies, participants were asked to rate their level of agreement/disagreement using a 7point Likert scale ( $1=$ completely disagree; $7=$ completely agree). Irvine 2012a calculated a composite score for each participant by computing the average response across the four test items. In both studies, study authors reported the group mean for participants in control and intervention groups before intervention and at followup eight weeks later. For the present analysis, the SMD in the change in mean between baseline and follow-up was compared for participants in intervention and control groups to assess effectiveness of the intervention.

\section{Adverse personal outcomes}

Needham 2005 examined the impact of the intervention in mitigating adverse outcomes of aggression using the Impact of Patient Aggression on Carers Scale (Needham 2005a). Each item on this 10-item instrument starts with the statement "After dealing with patient aggression ..." followed by a reaction that could occur following aggression (e.g. "I avoid contact with this patient"). Participants were instructed to give their response using a 5-point Likert scale indicating frequency of their reaction to aggression (i.e. "never," "rarely," "sometimes," "often," and "always"). This instrument was used to measure adverse outcomes before intervention and at follow-up 90 days later. Study authors reported the mean score on the three subscales (i.e. adverse moral reactions; adverse feelings to external sources; and impairment of the relationship between patient and carer) of this instrument for intervention and control group participants. For the present analyses, we selected "adverse moral reactions" as this reflected median scores across the three scales and fitted better the definition of personal outcome. We used the SMD in changes in this score between baseline and follow-up for control and intervention groups to assess the efficacy of an intervention in preventing or mitigating the adverse outcomes of aggression.

\section{Follow-up}

\section{Short term}

These included studies provided short-term follow-up: Anderson 2006 (six months); Fitzwater 2002 (two weeks); Irvine 2007 (one day); Irvine 2012a (eight weeks); Irvine 2012b (two weeks for aggression outcomes, eight weeks for other reported outcomes); Ming 2019 (three months); Needham 2005 (90 days); and Whittington 1996 (28 days).

\section{Medium term}

No included studies provided medium-term follow-up.

\section{Long term}

Only one study in the present review was considered to provide long-term follow-up (Arnetz 2000; one-year follow-up).

\section{Excluded studies}

Following screening of search results, review authors excluded 28 articles from the systematic review. Some studies were excluded due to research design considerations. Eleven studies were excluded because they did not include a control group (Beech 2006; Beech 2003; Cailhol 2007; Casalino 2015; Fernandes 2002; Gerdtz 2012; Gertz 1980; Lipscomb 2004a; Meehan 2006; PeekAsa 2002; Shah 1998). In addition, two studies were excluded because they included no control groups and the education/ training interventions were part of a multi-intervention program (Gillespie 2013; Gillespie 2014). One study was excluded because it did not have a concurrent control group, with control group participants selected after the intervention (Ore 2002). One study was excluded because control group measures were taken only once, as opposed to measures both before and after intervention (Ishak 2002).

One study was excluded because it used "a before and after study" research design with no comparison group (Adams 2017). One study was excluded because it was reported as an abstract duplicating other material (Kowalenko 2014). One publication was excluded because it described a grant application for a multi-program intervention, and its results were reported elsewhere (Gates 2013). Two studies were excluded because the education/training intervention was only one component of a multi-component intervention, and the contribution of education component effects could not be assessed in the analysis (Lipscomb 2004b; Lipscomb 2006).

Another reason for exclusion was lack of relevance of the study to the objective of the present study. One study was excluded because it investigated effects of an intervention aimed at patients rather than at healthcare workers (Cooper 2006). Another study was excluded because it investigated effects of an intervention on aggression between colleagues (Kang 2017). One study was excluded because its reported outcomes were not deemed relevant to the present review (Mclntosh 2003).

Finally, some studies were excluded because they provided no data pertaining to an intervention. Two studies were excluded because they did not report on an intervention (Kim 2018; Li 2018). One study was excluded because it did not report on an intervention and did not appear to provide any data (McElaney 2008). One paper was excluded because it was a literature review (Rittenmeyer 2013). Finally, one paper was rejected because it was a descriptive report rather than a report on an intervention study (Vousden 1987).

\section{Risk of bias in included studies}

The risk of bias of the included studies as assessed by the authors of this review is shown in Figure 2 and on an individual study basis in Figure 3. Details are provided in the section Characteristics of included studies. 
Figure 2. Figure 2. Review author's judgement about Risk of bias by Cochrane Collaboration's tool for assessing risk of bias within and across randomized trials. Risk of bias across studies.

Random sequence generation (selection bias)

Allocation concealment (selection bias)

Blinding of participants and personnel (performance bias): All outcomes

Blinding of outcome assessment (detection bias): All outcomes Incomplete outcome data (attrition bias): All outcomes

Selective reporting (reporting bias)

Other bias
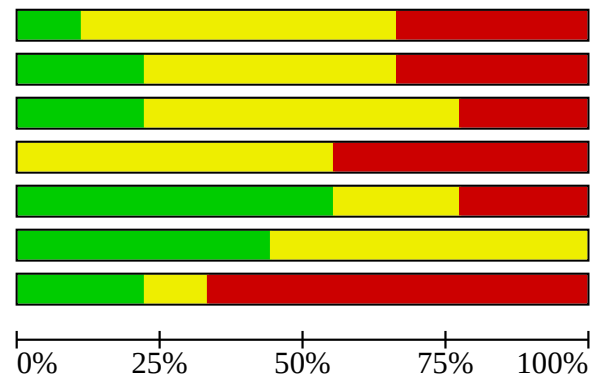
Figure 3. Figure 3. Review author's judgement about Risk of bias by Cochrane Collaboration's tool for assessing risk of bias within and across randomized trials. Risk of bias within studies.

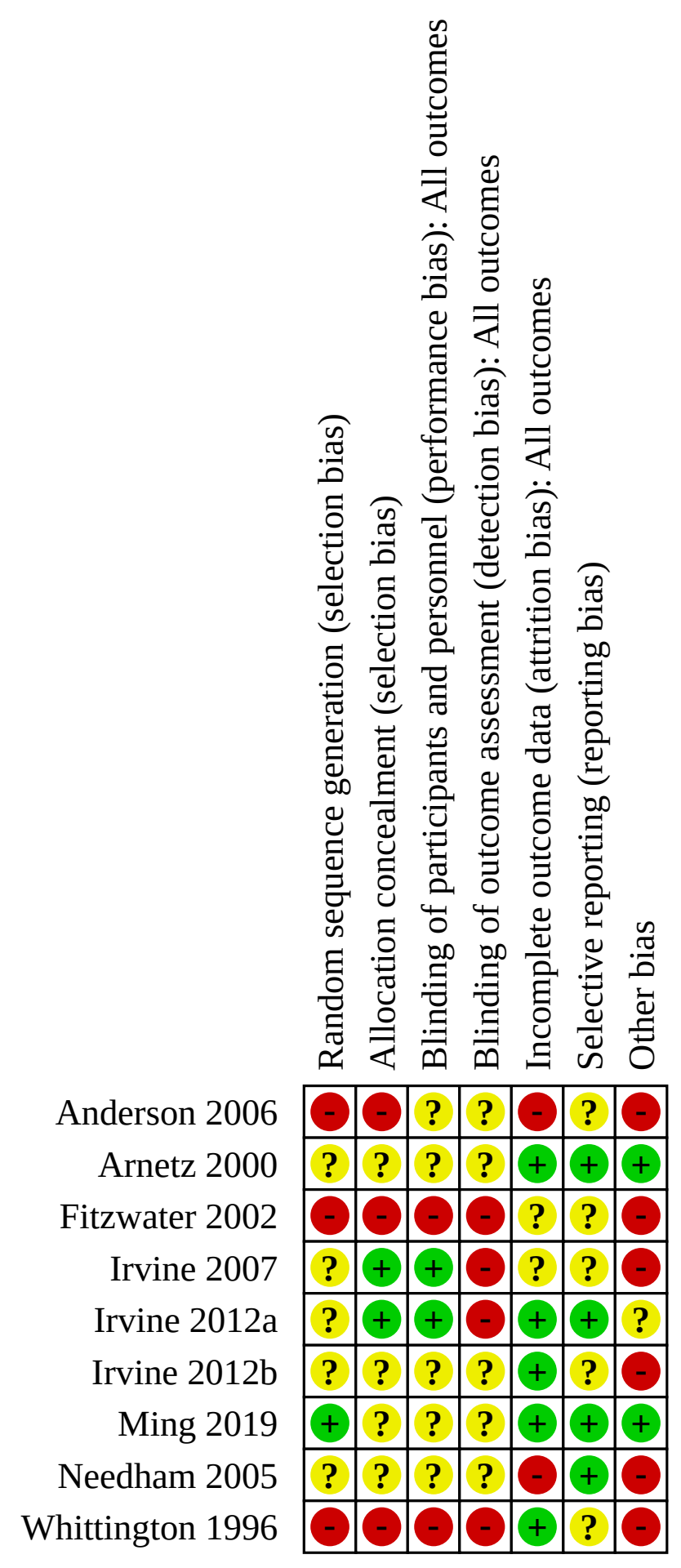




\section{Allocation}

Three studies were judged to have high risk of selection bias due to lack of allocation concealment (Anderson 2006; Fitzwater 2002; Whittington 1996). Two studies did not provide enough details about allocation concealment to allow determination of risk of selection bias (Arnetz 2000; Needham 2005). Finally, four studies were found to have taken enough precautions regarding allocation concealment to represent low risk of introducing bias (Irvine 2007; Irvine 2012a; Irvine 2012b; Ming 2019).

Three of the included studies were deemed to be at high risk for selection bias due to lack of random assignment (Anderson 2006; Fitzwater 2002; Whittington 1996). One study performed random assignment of participants in the experimental and control groups and thus was assessed to have low risk of selection bias (Ming 2019). The risk of selection bias due to lack of randomization was deemed unclear for the remaining included studies because they did not provide enough information to permit this assessment (Arnetz 2000; Irvine 2007; Irvine 2012a; Irvine 2012b; Needham 2005).

\section{Blinding}

\section{Performance bias}

Two studies were deemed to have high risk of performance bias due to lack of blinding of participants (Fitzwater 2002; Whittington 1996). Two studies did not blind participants regarding which cohort they belonged to, but we did not deem this to be a likely source of bias as education interventions were delivered online (Irvine 2007; Irvine 2012a). The other included studies did not provide enough information to permit determination of the risk of performance bias (Anderson 2006; Arnetz 2000; Irvine 2012b; Ming 2019; Needham 2005).

The research design of the included studies involved comparing those who underwent intervention with those who did not. As a result, there was no blinding of the person(s) giving the intervention in face-to-face interventions (Arnetz 2000; Fitzwater 2002; Ming 2019; Needham 2005; Whittington 1996). Lack of blinding of research personnel in studies that used an online intervention was not deemed an issue, given the absence of interactions between people delivering the intervention and those receiving it (Anderson 2006; Irvine 2007; Irvine 2012a; Irvine 2012b).

\section{Detection bias}

The included studies relied primarily on self-assessment to determine the effects of education and training interventions on outcome measures, thereby potentially inserting a source of bias inherent to these methods. For instance, participants in Arnetz 2000 were asked questions regarding changes in their awareness of high-risk situations of aggression. A number of other factors may contribute to a report on increased awareness, including underestimation of one's prior awareness, overestimation of one's awareness at the time responses were provided, and social desirability bias leading participants to respond in a manner that would be viewed favorably. This poses a significant risk of bias for studies in which participants were not blinded (Fitzwater 2002; Irvine 2007; Irvine 2012a; Whittington 1996). The risk of bias for the remaining studies was deemed unclear due to insufficient information about blinding (Anderson 2006; Arnetz 2000; Irvine 2012b; Ming 2019; Needham 2005).

\section{Incomplete outcome data}

Five studies detailed loss of participants and were deemed to be at low risk of attrition bias (Arnetz 2000; Irvine 2012a; Irvine 2012b; Ming 2019; Whittington 1996). Two studies reported high attrition rates and thus were determined to be at high risk for attrition bias (Anderson 2006; Needham 2005). Finally, two studies did not provide sufficient information to permit assessment of the risk of attrition bias (Fitzwater 2002; Irvine 2007).

\section{Selective reporting}

Four studies were judged to have low risk of reporting bias (Arnetz 2000; Irvine 2012a; Ming 2019; Needham 2005). The risk of selective reporting bias was deemed unclear for the remainder of the studies due to insufficient information to permit judgment (Anderson 2006; Fitzwater 2002; Irvine 2007; Irvine 2012b; Whittington 1996).

\section{Other potential sources of bias}

Categorization of interventions based on duration and types of delivery may have an impact on study results as they are considered to have the same potential for effect. The categorization of followup may also hinder time to detect differences between short- and long-term effects.

\section{Effects of interventions}

See: Summary of findings 1 Summary of findings

\section{Education only}

No studies reported on an education only program.

\section{Education combined with training \\ Primary outcome-episodes of aggression \\ Short-term follow-up CRCTs}

Evidence provided by CRCTs was very uncertain concerning effects of education and training on episodes of aggression at short-term follow-up. Results of two CRCTs were combined in a meta-analysis (Fitzwater 2002; Irvine 2012b), which did not show a statistically significant effect of the intervention on the number of episodes of aggression (SMD $-0.33,95 \% \mathrm{Cl}-1.27$ to 0.61 ; Analysis 1.1). No significant subgroup differences between these studies based on type and duration of the intervention were found $\left(P=0.18 ; I^{2}\right.$ $=44.4 \%)$.

\section{Short-term follow-up CBAs}

The two CBAs provided very uncertain evidence about effects of education and training on the number of workers reporting episodes of aggression at short-term follow-up. Whittington 1996 did not find a statistically significant effect of intervention on the risk ratio of aggression against workers (RR 2.30, 95\% Cl 0.97 to 5.42; Analysis 1.2).

Anderson 2006 reported a significant reduction in the mean number of reported episodes of aggression at follow-up (SMD -1.24, $95 \% \mathrm{Cl}-2.16$ to -0.33 ; Analysis 1.3 ).

\section{Long-term follow-up (CRCTs)}

Low-certainty evidence suggests that education and training does not reduce the number of workers reporting episodes of aggression at short-term follow-up. A long-term follow-up CRCT revealed no 
statistically significant effect of an extended face-to-face education intervention on the probability of reporting being the target of aggression (RR 1.14, 95\% Cl 0.95 to 1.37; Analysis 1.4) (Arnetz 2000).

\section{Secondary outcome-personal knowledge about aggression}

\section{Short-term follow-up (RCT)}

See Analysis 2.1.

Low-certainty evidence suggests that education and training interventions improved knowledge about aggression at short-term follow-up. One RCT reported short-term follow-up data regarding knowledge about aggression following an online education intervention (Irvine 2007). Analysis revealed a statistically significant effect favoring the intervention group (SMD 0.86, 95\% Cl 0.34 to 1.38$)$.

\section{Long-term follow-up (CRCT)}

See Analysis 2.2

Low-certainty evidence suggests that education and training interventions did not improve knowledge about aggression at longterm follow-up. Arnetz 2000 assessed the impact of an education intervention on enhancing awareness of risk situations and found no statistically significant effect of training on personal knowledge about aggression (RR $1.26,95 \% \mathrm{Cl} 0.90$ to 1.75 ).

\section{Secondary outcome-attitudes}

\section{Short-term follow-up (RCT/CRTC)-general attitudes}

See Analysis 3.1.

Very low-quality evidence suggests that education/training interventions improved attitudes among healthcare workers at short-term follow-up. Two CRTCs and three RCTs measured effects of education interventions on the attitudes of participants toward patient aggression in short-term follow-up (Irvine 2007; Irvine 2012a; Irvine 2012b; Ming 2019; Needham 2005). Results of the meta-analysis revealed a statistically significant small effect on attitudes favoring the education group (SMD $0.59,95 \% \mathrm{Cl} 0.24$ to 0.94).

Subgroup differences were statistically significant $\left(P<0.001 ; I^{2}\right.$ $=78.3 \%$ ), suggesting that type and duration of an intervention accounted for different effects. Ming 2019 found a statistically significant moderate effect favoring the education group (SMD 0.78, $95 \% \mathrm{Cl} 0.58$ to 0.99 ). Irvine 2007 found a statistically significant large effect favoring the education group (SMD 1.23, 95\% Cl 0.69 to 1.78). Irvine $2012 \mathrm{a}$ and Irvine $2012 \mathrm{~b}$ found a statistically significant small effect favoring the education group (SMD 0.33, 95\% Cl 0.05 to 0.61 ). Needham 2005 found no statistically significant effect of intervention on attitudes toward aggression (SMD $-0.03,95 \% \mathrm{Cl}$ -0.68 to 0.73$)$.

\section{Secondary outcome-skills}

\section{Short-term follow-up (RCT/CRCT)-empathy}

See Analysis 4.1.

Very low-quality evidence suggests that healthcare workers who underwent education interventions did not show more empathy than those in the control group at follow-up. One RCT and one CRCT assessed the impact of an online education intervention on workers' empathy toward patients (Irvine 2012a; Irvine 2012b). Combined results of short-term follow-up revealed a small effect favoring the intervention that was not statistically significant (SMD $0.21,95 \% \mathrm{Cl}-0.07$ to 0.49 ).

\section{Secondary outcome-adverse impact \\ Short-term follow-up (CRCT)-adverse personal impact}

See Analysis 5.1.

Very low-quality evidence suggests that education and training interventions did not help mitigate the adverse outcomes of patient aggression for healthcare workers. One CRCT tested the impact on adverse moral reactions at short-term follow-up (Needham 2005). These results revealed a small negative effect in favor of the control group that was not statistically significant (SMD $-0.31,95 \% \mathrm{Cl}-1.02$ to 0.40$)$.

\section{DISCUSSION}

We included nine studies-four cluster-randomized controlled trials (CRCTs), three randomized controlled trials (RCTs), and 2 controlled before and after studies (CBAs) -with a total of 1688 participants. Five studies reported episodes of aggression, and six studies reported secondary outcomes. Seven studies were conducted among nurses or nurse aides, and two studies among healthcare workers in general. Three studies took place in longterm care, two in the psychiatric ward, and four in hospitals or health centers. Studies were reported from the United States, Switzerland, the United Kingdom, Taiwan, and Sweden.

All included studies reported on education combined with training interventions. Four studies evaluated online programs, and five evaluated face-to-face programs. Five studies were of long duration (up to 52 weeks), and four studies were of short duration. Eight studies provided short term follow-up ( $<3$ months) and one study long-term follow-up (> 1 year). Seven studies were rated as being at "high" risk of bias in multiple domains, and all had "unclear" risk of bias in a single or multiple domains. Effects of education and training interventions in aggression prevention in healthcare settings are shown in Summary of findings 1.

\section{Summary of main results}

\section{Effects of education and training interventions in aggression prevention on reduction of episodes of aggression}

The evidence is very uncertain about effects of education and training interventions on aggression, compared to no intervention, at short-term follow-up (standardized mean difference [SMD] 0.33, 95\% confidence interval [CI] -1.27 to $0.61,2$ CRCTs; risk ratio [RR] 2.30, $95 \% \mathrm{Cl} 0.97$ to $5.4,1 \mathrm{CBA}$; SMD $-1.24,95 \% \mathrm{Cl}-2.16$ to -0.33 , 1 CBA; very low-quality evidence). Education may not have an effect on aggression compared to no intervention in the long term (RR $1.14,95 \% \mathrm{Cl} 0.95$ to $1.37,1 \mathrm{RCT}$; low-quality evidence). The primary outcome findings of this review may be explained by different factors. First, increased knowledge about aggression may lead to increased awareness of aggression incidents, thereby offsetting actual reductions in aggression. As such, participants may have been more willing to report these episodes, which are normally under-reported (Arnetz 2015). Further, the self-reported nature of data on the number of aggression incidents in some studies complicates interpretation, as it is unclear whether the increases are due to greater awareness of aggression or willingness 
to report it, or whether they represent a genuine increase in the number of events of aggression. Another possible explanation is that training healthcare workers in aggression management and raising their self-efficacy in dealing with such situations renders them more willing to engage in, instead of avoiding, situations in which there is high risk of aggression. Second, the heterogeneity of the ways in which episodes of aggression were recorded and reported made it impossible to combine all included studies into a single measure. Moreover, the use of dichotomous measures in some included studies may not have captured effects of the intervention, as they represent only the proportion of healthcare workers who experienced aggression before and after the intervention-not the reduction or increase in the number of episodes of aggression for each participant. For example, a participant may experience fewer episodes of aggression at followup compared to baseline but would still answer "yes" if asked a dichotomous question, thereby not reducing the proportion of workers who experience these acts. Third, the statistical power of certain studies may not have been sufficient to reject the null hypothesis. Thus, we cannot determine whether education and training interventions in aggression prevention result in reduction of episodes of aggression toward healthcare workers.

\section{Effects of education and training interventions in aggression prevention on secondary outcomes}

Education may result in increased personal knowledge about workplace aggression at short-term follow-up (SMD 0.86, 95\% CI 0.34 to $1.38,1 \mathrm{RCT}$; low-quality evidence) but may not be effective at long-term follow-up (RR $1.26,95 \% \mathrm{Cl} 0.90$ to $1.75,1 \mathrm{RCT}$; very low-quality evidence). Education may improve attitudes among healthcare workers at short-term follow-up, but the evidence is very uncertain (SMD 0.59, 95\% Cl 0.24 to $0.94,2$ CRCTs and 3 RCTs; very low-quality evidence) and the type and duration of interventions produced effects of different sizes. The evidence is very uncertain about effects on skills related to workplace aggression (SMD 0.21 , $95 \% \mathrm{Cl}-0.07$ to $0.49,1 \mathrm{RCT}$ and $1 \mathrm{CRCT}$; very low-quality evidence) or on adverse personal outcomes (SMD $-0.31,95 \% \mathrm{Cl}-1.02$ to $0.40,1 \mathrm{RCT}$; very low-quality evidence). Still, the heterogeneity of measurements of the concepts made it impossible to combine outcomes in a single measure, and the statistical power for some secondary outcomes was low.

\section{Overall completeness and applicability of evidence}

The search strategy that we used to find studies for the present review sought to detect as many relevant articles as possible. To do this, we used several terms that are semantically close or related to aggression, such as violence and assault. We also did not restrict the search to any single category of healthcare worker nor filter out search results based on language. We included several types of research design assessing effects of education and training interventions that allowed comparison between those who received intervention and those who did not. This included RCTs, CRCTs, and CBAs. We also searched a wide array of databases to maximize the number of hits. This search returned 4744 potential articles for us to screen for the present review. We pursued additional search results from the reference sections of retrieved papers and sought papers from experts in the field. Given this search strategy, we are confident that we did not miss many studies investigating the outcomes of education and training interventions for dealing with aggression among healthcare workers.
Participant occupations included certified nurse assistants or nurse aides (Fitzwater 2002; Irvine 2007; Irvine 2012a; Irvine 2012b), nurses (Needham 2005; Ming 2019; Whittington 1996), and non-specific healthcare workers (Anderson 2006; Arnetz 2000). The settings in which these studies took place include a small community hospital (Anderson 2006), psychiatric wards (Needham 2005; Whittington 1996), long-term care facilities or nursing homes (Fitzwater 2002; Irvine 2007; Irvine 2012a; Irvine 2012b), a medical center (Ming 2019), and healthcare workplaces (Arnetz 2000). All of these studies were conducted in highincome countries, namely, Great Britain, the United States, Taiwan, and Sweden. Thus, several healthcare workers were missing in studies from low- and middle-income countries, as were other types of healthcare workers such as physicians and first aid workers.

Our search failed to find any study investigating organizational outcomes such as absenteeism or employee turnover. Further, the studies on individual outcomes focused on variables such as knowledge, attitudes, and self-efficacy regarding aggression. No study was found that investigated effects of intervention on physical and mental health issues, nor professional difficulties.

Another obstacle to the generalizability of review findings is the fairly short follow-up period reported by most of the included studies. Consequently, caution must be exercised in extrapolating the long-term impact of education and training programs in aggression prevention based on currently available data.

\section{Quality of the evidence}

We found the quality of evidence for primary and secondary outcomes to be very low to low due to the bias implicit in self-reporting and the heterogeneity of outcome measurement approaches across the small body of included studies. In light of the very low to low quality of the existing research, additional research findings from high-quality studies are likely to have a significant impact on our confidence related to the effects of education and training programs in aggression prevention and minimization.

\section{Potential biases in the review process}

One of the limitations of this review is the scarcity of studies on the topic, as the lack of studies did not allow us to perform sensitivity analyses to evaluate optimal intervention parameters (e.g. face-to-face, online, duration) to reduce the frequency of episodes of aggression and mitigate their impact. Adjustment of the sample size in CRCTs reduced the statistical power of the analysis, thereby increasing the chance of type II error. Effects on episodes of aggression became insignificant for Fitzwater 2002 and Irvine $2012 b$, and effects on personal knowledge became insignificant for Arnetz 2000.

Categorization of interventions based on duration and type of delivery may also have biased this review and impacted the results. Because we found no evidence on differences in effectiveness of online or face-to-face programs, short to long duration, or single to multiple sessions, we started with the assumption that they have the same effect on our outcomes. Still, we computed subgroups to assess differences according to duration and type of delivery. In the same vein, our categorization of follow-up may have hindered our capacity to detect differences between short-term and long-term effects. 


\section{Agreements and disagreements with other studies or reviews}

Our search revealed one review related to the effectiveness of education and training for preventing workplace aggression in healthcare settings (Rittenmeyer 2013). The scope of the review was limited to workplace aggression between healthcare workers. We did not discover any studies that examined the effectiveness of interventions aimed at preventing workplace violence or ameliorating its effect.

\section{AUTHORS' CONCLUSIONS}

\section{Implications for practice}

- Education may not have an effect or may have an inconsistent effect on preventing workplace aggression, compared to no intervention, at short-term follow-up on prevention

- Education may not have an effect on preventing workplace aggression compared to no intervention in the long term

- Education may increase healthcare workers' personal knowledge about patient aggression

- Education may increase healthcare workers' positive attitudes toward patient aggression

- Education may not have an effect on skills related to patient aggression

- Education may not have an effect on adverse personal outcomes of patient aggression

\section{Implications for research}

According to the PICO framework, future studies on education and training interventions that aim to prevent and minimize workplace aggression directed toward healthcare workers by patients and patient advocates should:

- focus on specific settings of healthcare work where exposure to patient aggression is high (e.g. mental health workers). As such, a reduction in episodes of aggression following training may be significant only for workers who are highly exposed. Moreover, as most studies have assessed episodes of aggression toward nurses, future studies should include other types of healthcare workers who are also victims of aggression in the same settings, such as orderlies (healthcare assistants);

- benefit from the ability to track reports of aggression at an institutional level and rely on multi-source data (combining subjective and objective measures) and validated measures. Studies should also incorporate an active comparison to prevent raising awareness and reporting in the intervention group only;
- specify the duration and type of delivery to provide insight as to the method that is most effective to reduce episodes of aggression against healthcare workers; and

- incorporate variables such as days lost to sick leave and employee turnover into their outcome measures. In addition, care should be taken to study the longer-term outcomes of these education and training programs. Further, given that several interventions did not specify their assessment tools, future studies should endeavor to disclose their instruments and use the same well-established, validated questionnaires. In addition, measures of episodes of aggression should be reported as frequencies rather than as the number or proportion of workers who have been the target of aggression, as the former is more sensitive due to the fact that a worker can experience multiple episodes of aggression over an assessment period.

\section{ACKN OWLEDGEMENTS}

We wish to acknowledge the contributions of Dr Maya Guest, formerly of the University of Newcastle, Australia (now retired), who originally conceived the protocol and led its early development, including in relation to the search strategy design, in conjunction with the University of Newcastle librarians; and the contributions of Associate Professor Ashley Kable and Professor Michael Hazelton from the University of Newcastle, Australia, to early development of the protocol.

We thank Jani Ruotsalainen, former Managing Editor of the Cochrane Work Review Group, for providing administrative and logistical support for the conduct of the current review; and Kaisa Neuvonen, Trials Search Co-ordinator, Cochrane Work Review Group, for developing and testing the search strategies, which were subsequently employed for the conduct of this review.

We acknowledge the contributions of Murray Turner, Librarian, University of Canberra, in completing the initial search of the literature. We also acknowledge the contributions of Catelyn Richards, Research Assistant, Nursing and Midwifery; and Anne Young, Librarian; both of Monash University, for their efforts in updating the literature search to 31 March 2018. We additionally thank Dr Louisa Lam, Deputy Dean, Federation University, for assistance in translating a Chinese language manuscript for the review paper.

We would also like to thank the Cochrane Work Review Group's Coordinating Editor Jos Verbeek, Managing Editor Julitta Boschman, and Editors Thais Morata and Anneli Ojajärvi and external peer referees Joy Duxbury and Sabine Hahn for their comments. Last but not least, we thank copy editor Dolores Matthews and plain language summary writer Carolyn Hughes for copyediting the text. 


\section{RE F E R E N C E S}

\section{References to studies included in this review}

Anderson 2006 \{published data only\}

Anderson C. Training efforts to reduce reports of workplace violence in a community health care facility. Journal of Professional Nursing 2006;22(5):289-95. [DOI: 10.1016/ j.profnurs.2006.07.007]

Arnetz 2000 \{published data only\}

* Arnetz JE, Arnetz BB. Implementation and evaluation of a practical intervention programme for dealing with violence towards health care workers. Journal of Advanced Nursing 2000;3(3):668-80.

\section{Fitzwater 2002 \{published data only\}}

Fitzwater EL, Gates DM. Testing an intervention to reduce assaults on nursing assistants in nursing homes: a pilot study. Geriatric Nursing 2002;23(1):18-23.

Irvine 2007 \{published data only\} Irvine AB, Bourgeois M, Billow M, Seeley JR. Internet training for nurse aides to prevent resident aggression. Journal of the American Medical Directors Association 2007;8(8):519-26.

Irvine 2012a \{published data only\}

Irvine AB, Billow MB, Gates DM, Fitzwater EL, Seeley JR, Bourgeois $\mathrm{M}$. Internet training to respond to aggressive resident behaviors. The Gerontologist 2012;52(1):13-23. [DOI: 10.1093/ geront/gnr069]

Irvine 2012b \{published data only\}

Irvine B, Billow MB, Gates DM, Fitzwater EL, Seeley JR, Bourgeois M. An internet training to reduce assaults in longterm care. Geriatric Nursing 2012;33(1):28-40.

\section{Ming 2019 \{published data only\}}

Ming JL, Tseng LH, Huang HM, Hong SP, Chang Cl, Tung CY. Clinical simulation teaching program to promote the effectiveness of $n$ [臨床情境模擬教學促進護理人員因應職場暴力之成效研究]. Journal of Nursing 2019;66(3):59-71.

\section{Needham 2005 \{published data only\}}

Needham I, Abderhalden C, Halfens RJ, Dassen T, Haug HJ, Fischer JE. The effect of a training course in aggression management on mental health nurses' perceptions of aggression: a cluster randomised controlled trial. International Journal of Nursing Studies 2005;42(6):649-55. [DOI: 10.1016/ j.ijnurstu.2004.10.003]

\section{Whittington 1996 \{published data only\}}

Whittington R, Wykes T. An evaluation of staff training in psychological techniques for the management of patient aggression. Journal of Clinical Nursing 1996;5(4):257-61.

\section{References to studies excluded from this review}

\section{Adams 2017 \{published data only\}}

Adams J, Knowles A, Irons G, Roddy A, Ashworth J. Assessing the effectiveness of clinical education to reduce the frequency and recurrence of workplace violence. Australian Journal of Advanced Nursing 2017;34(3):6-15.

Beech 2003 \{published data only\}

Beech B, Leather P. Evaluating a management of aggression unit for student nurses. Journal of Advanced Nursing 2003;44(6):603-12.

Beech 2008 \{published data only\}10.1016/j.nepr.2007.04.004 Beech B. Aggression prevention training for student nurses: differential responses to training and the interaction between theory and practice. Nurse Education in Practice 2008;8(2):94-102. [DOI: 10.1016/j.nepr.2007.04.004]

Cailhol 2007 \{published data only\}10.1016/

j.genhosppsych.2006.10.007

Cailhol L, Allen M, Moncany AH, Cicotti A, Virgillito S, Barbe RP, et al. Violent behavior of patients admitted in emergency following drug suicidal attempt: a specific staff educational crisis intervention. General Hospital Psychiatry 2007;29(1):42-4. [DOI: 10.1016/j.genhosppsych.2006.10.007]

Casalino 2015 \{published data only\}

Casalino E, Choquet C, Thomas S, Erhel S, Cossard P. Violence in the emergency department: evaluation of a policy to reduce violence in one university emergency department in the Paris area. Annales Francaises de Medecine d'Urgence 2015;5(4):226-37.

Cooper 2006 \{published data

only\}10.1097/01.ta.0000236576.81860.8c

Cooper C, Eslinger DM, Stolley PD. Hospital-based violence intervention programs work. Journal of Trauma 2006;61(3):534-7. [DOI: 10.1097/01.ta.0000236576.81860.8c]

Fernandes 2002 \{published data only\} Fernandes CM, Raboud JM, Christenson JM, Bouthillette F,

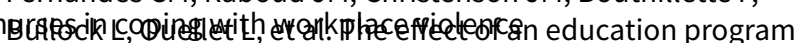
on violence in the emergency department. Annals of Emergency Medicine 2002;39(1):47-55.

Gates 2013 \{published data only\}

Gates DM. A multi-site intervention to reduce violence in hospital emergency departments: final grant report. Grant Number R01-OH-009544. National Institute for Occupational Safety and Health 2013. [http://www.cdc.gov/niosh/ nioshtic-2/20045211.html]

Gerdtz 2012 \{published data only\}

Gerdtz MF, Daniel C, Dearie V. An evaluation of a novel training program for preventing occupational violence in emergency departments. Abstract from the 14th International Conference on Emergency Medicine. Academic Emergency Medicine 2012;19(6):751-2.

\section{Gertz 1980 \{published data only\}}

Gertz B. Training for prevention of assaultive behavior in a psychiatric setting. Hospital and Community Psychiatry 1980;31(9):628-30. 
Gillespie 2013 \{published data only\}10.1016/j.jen/2012.12.010

Gillespie GL, Gates DM, Mentzel T, Al-Natour A, Kowalenko T. Evaluation of a comprehensive ED violence prevention program. Journal of Emergency Nursing 2013;39(4):376-83. [DOI: 10.1016/j.jen/2012.12.010]

Gillespie 2014 \{published data only\}10.1016/j.jen.2014.01.003 Gillespie GL, Gates DM, Kowalenko T, Bresler S, Succop P. Implementation of a comprehensive intervention to reduce physical assaults and threats in the emergency department. Journal of Emergency Nursing 2014;40(6):586-91. [DOI: 10.1016/ j.jen/2014.01.003]

\section{Ishak 2002 \{published data only\}}

Ishak M, Christensen M. Achieving a better management for patients' aggressive behaviour: evaluation of a training program. Journal of Occupational Health and Safety-Australia and New Zealand 2002;18(3):231-7.

\section{Kang 2017 \{published data only\}}

Kang J, Kim JI, Yun S. Effects of a cognitive rehearsal program on interpersonal relationships, workplace bullying, symptom experience, and turnover intention among nurses: a randomized controlled trial. Journal of Korean Academy of Nursing 2017;47(5):689-99.

\section{Kim 2018 \{published data only\}}

Hyejin K, Ji-Su K, Kwisoon C, Yeunhee K, Jae-seok S. Mediating effects of workplace violence on the relationships between emotional labour and burnout among clinical nurses. Journal of Advanced Nursing 2018;74:2331-9.

\section{Kowalenko 2014 \{published data only\}}

Kowalenko T, Gates D, Gillespie G, Succop P. Implementation of a comprehensive intervention to violence against health care workers in the emergency department. Abstract from the 2014 Society for Academic Emergency Medicine Annual Meeting. In: Academic Emergency Medicine. Vol. 21(Suppl S1). 2014:S306

\section{Li 2018 \{published data only\}}

Li P, Xing K, Qiao H, Fang H, Ma H, Jiao M, et al. Psychological violence against general practitioners and nurses in Chinese township hospitals: incidence and implications. Health and Quality of Life Outcomes 2018;16(1):117-26.

\section{Lipscomb 2004a \{published data only\}}

Lipscomb JA, Rosen J, McPhaul K. Violence prevention in the mental healthcare setting: evaluation of an intervention based on OSHA guidelines. In: Presentation to the American Public Health Association 132nd Annual Meeting and Exposition, Washington, DC. 2004. [http://apha.confex.com/apha/132am/ techprogram/paper_88020.htm]

\section{Lipscomb 2004b \{published data only\}}

Lipscomb JA, Soeken K, Rosen J, McPaul K, Choi M, Geiger B, et al. Evaluation of OSHA violence prevention guidelines in mental health. Final report. Grant-Number-R01-OH-004051. National Institute for Occupational Safety and Health 2004. [http://www.cdc.gov/niosh/nioshtic-2/20029678.html]

\section{Lipscomb 2006 \{published data only\}}

Lipscomb J, McPhaul K, Rosen J, Brown JG, Choi M, Soeken K, et al. Violence prevention in the mental health setting: the New York State experience. Canadian Journal of Nursing Research 2006;38(4):96-117.

\section{McElaney 2008 \{published data only\}}

McElaney LA. Workplace violence training for nurses. Final report. Grant-Number-R43-OH-009180. National Institute for Occupational Safety and Health 2008. [http://www.cdc.gov/ niosh/nioshtic-2/20044849.html]

\section{McIntosh 2003 \{unpublished data only\}}

Mclntosh D. Testing an intervention to increase self-efficacy of staff in managing clients perceived as violent [PhD thesis].. Cincinnati (USA): University of Cincinnati, 2003.

\section{Meehan 2006 \{published data only\}}

Meehan T, Fjeldsoe K, Stedman T, Duraiappah V. Reducing aggressive behaviour and staff injuries: a multi-strategy approach. Australian Health Review 2006;30(2):203-10.

\section{Ore 2002 \{published data only\}}

Ore T. Workplace assault management training: an outcome evaluation. Journal of Healthcare Protection Management 2002;18(2):61-93.

\section{Peek-Asa 2002 \{published data only\}}

Peek-Asa C, Cubbin L, Hubbell K. Violent events and security programs in California emergency departments before and after the 1993 Hospital Security Act. Journal of Emergency Nursing 2002;28(5):420-78.

\section{Rittenmeyer 2013 \{published data only\}}

* Rittenmeyer L, Huffman D, Hopp L, Block M. A comprehensive systematic review on the experience of lateral/horizontal violence in the profession of nursing. $J B I$ Database of Systematic Reviews and Implementation Reports 2013;11(11):362-468

\section{Shah 1998 \{published data only\}}

Shah A, De T. The effect of an educational intervention package about aggressive behaviour directed at the nursing staff on a continuing care psychogeriatric ward. International Journal of Geriatric Psychiatry 1998;13(1):35-40.

Vousden 1987 \{published data only\}

Vousden M. Are you safe? Nursing Times 1987;83(26):28-30.

\section{Additional references}

\section{Anderson 2010}

Anderson L, FitzGerald M, Luck L. An integrative literature review of interventions to reduce violence against emergency department nurses. Journal of Clinical Nursing 2010;19(17-18):2520-30.

\section{Arimatsu 2008}

Arimatsu M, Wada K, Yoshikawa T, Oda S, Taniguchi H, Aizawa Y, et al. An epidemiological study of work-related 
violence experienced by physicians who graduated from a medical school in Japan. Journal of Occupational Health 2008;50(4):357-61.

\section{Arnetz 2001}

Arnetz JE, Arnetz BB. Violence toward health care staff and possible effects on the quality of patient care. Social Science \& Medicine 2001;52(3):417-27.

\section{Arnetz 2015}

Arnetz JE, Hamblin L, Ager J, Luborsky M, Upfal MJ, Russell J, et al. Underreporting of workplace violence: comparison of self-report and actual documentation of hospital incidents. Workplace Health \& Safety 2015;63(5):200-10. [DOI: https:// doi.org/10.1177/2165079915574684]

\section{Atawneh 2003}

Atawneh F, Zahid M, Al-Sahlawi K, Shahid A, Al-Farrah M. Violence against nurses in hospitals: prevalence and effects. British Journal of Nursing 2003;12(2):102-7.

\section{Beech 2006}

Beech B, Leather P. Workplace violence in the health care sector: a review of staff training and integration of training evaluation models. Aggression and Violent Behavior: A Review Journal 2006;11(1):27-43. [DOI: 10.1016/j.avb.2005.05.004]

\section{Bowers 2006}

Bowers L, Nijman H, Allan T, Simpson A, Warren J, Turner LR. Prevention and management of aggression training and violent incidents on UK acute psychiatric wards. Psychiatric Services 2006;57(7):1022-6.

\section{Briggs 2003}

Briggs F, Broadhurst D, Hawkins R. Violence, threats and intimidation in the lives of professionals whose work involves child protection. A report on a research project funded by the Australian Criminology Research Council. CRC 15/01-02, 2003. crg.aic.gov.au/reports/200102-15.pdf (accessed August 2, 2015).

\section{Brown 2011}

Brown LP, Rospenda KM, Sokas RK, Conroy L, Freels S, Swanson NG. Evaluating the association of workplace psychosocial stressors with occupational injury, illness, and assault. Journal of Occupational and Environmental Hygiene 2011;8(1):31-7.

\section{Bulatao 1996}

Bulatao EQ, VandenBos GR. Workplace violence: its scope and issues. In: VandenBos GR, Bulatao EQ, editors(s). Violence on the Job: Identifying Risks and Developing Solutions. Washington, DC: American Psychological Association, 1996:1-23.

\section{Camerino 2008}

Camerino D, Estryn-Behar M, Conway PM, van Der Heijden BIJM, Hasselhorn $\mathrm{H}-\mathrm{M}$. Work-related factors and violence among nursing staff in the European NEXT study: a longitudinal cohort study. International Journal of Nursing Studies 2008;45(1):35-50.

\section{Campbell 2001}

Campbell MK, Mollison J, Grimshaw JM. Cluster trials in implementation research: estimation of intracluster correlation coefficients and sample size. Statistics in Medicine 2001;20(3):391-9.

\section{Campbell 2011}

Campbell JC, Messing JT, Kub J, Agnew J, Fitzgerald S, Fowler B, et al. Workplace violence: prevalence and risk factors in the safe at work study. Journal of Occupational \& Environmental Medicine 2011;53(1):82-9.

\section{Carluccio 2010}

Carluccio A, Knychala V, Marshall C. Violence against frontline NHS staff: research study conducted for $\mathrm{COI}$ on behalf of the NHS Security Management Service. London: NHS Security Management Service, 2010.

\section{Ceramidas 2010}

Ceramidas DM, Parker R. A response to patient-initiated aggression in general practice: Australian professional medical organisations face a challenge. Australian Journal of Primary Health 2010;16(3):252-9.

\section{Chappell 2006}

Chappell D, di Martino V. Violence at Work. 3rd edition. Geneva: International Labour Office, 2006.

\section{Child 2010}

Child RJ, Mentes JC. Violence against women: the phenomenon of violence against nurses. Issues in Mental Health Nursing 2010;31(2):89-95. [DOI: 10.3109/01612840903267638]

\section{Cookson 2012}

Cookson H, Buckley P. Violence at Work: Findings From the 2010/11 British Crime Survey. London: Health Service Executive, 2012.

\section{di Martino 2002}

di Martino V. Workplace violence in the health sector. Country case studies in Brazil, Bulgaria, Lebanon, Portugal, South Africa, Thailand and an additional Australian study. Synthesis report. Geneva: International Labour Office, International Council of Nurses, World Health Organization and Public Services International. Joint Programme on Workplace Violence in the Health Sector, 2002.

\section{di Martino 2005}

di Martino V. A cross-national comparison of workplace violence and response strategies. In: Bowie V, Fisher BS, Cooper CL, editors(s). Workplace Violence: Issues, Trends, Strategies. Cullompton, UK: Willan Publishing, 2005:15-36.

\section{Downs 1998}

Downs SH, Black N. The feasibility of creating a checklist for the assessment of the methodological quality both of randomised and non-randomised studies of health care interventions. Journal of Epidemiology and Community Health 1998;52(6):377-84. 


\section{Dupré 2014}

Dupré KE, Dawe KA, Barling J. Harm to those who serve: effects of direct and vicarious customer-initiated workplace aggression. Journal of Interpersonal Violence 2014;29(13):2355-77. [DOI: 10.1177/0886260513518841]

\section{Duxbury 2002}

Duxbury J. An evaluation of staff and patient views of and strategies employed to manage inpatient aggression and violence on one mental health unit: a pluralistic design. Journal of Psychiatric and Mental Health Nursing 2002;9(3):325-37.

\section{Duxbury 2008}

Duxbury J, Hahn S, Needham I, Pulsford D. The Management of Aggression and Violence Attitude Scale (MAVAS): a crossnational comparative study. Journal of Advanced Nursing 2008;62(5):596-606. [DOI: 10.1111/j.1365-2648.2008.04629.x]

\section{Estrada 2010}

Estrada F, Nilsson A, Jerre K, Wikman S. Violence at work-the emergence of a social problem. Journal of Scandinavian Studies in Criminology and Crime Prevention 2010;11(1):46-65.

\section{Farrell 2005}

Farrell G, Cubit K. Nurses under threat: a comparison of content of 28 aggression management programs. International Journal of Mental Health Nursing 2005;14:44-53.

\section{Farrell 2006}

Farrell GA, Bobrowski C, Bobrowski P. Scoping workplace aggression in nursing: findings from an Australian study. Journal of Advanced Nursing 2006;55(6):778-87.

\section{Farrell 2010}

Farrell GA, Shafiei T, Salmon P. Facing up to 'challenging behaviour': a model for training in staff-client interaction. Journal of Advanced Nursing 2010;66(7):1644-55.

\section{Flannery 2001}

Flannery RB. The employee victim of violence: recognizing the impact of untreated psychological trauma. American Journal of Alzheimer's Disease and Other Dementias 2001;16(4):230-3.

\section{Frank 1998}

Frank E, Brogan D, Schiffman M. Prevalence and correlates of harassment among US women physicians. Archives of Internal Medicine 1998;158(4):352-8.

\section{Gascón 2009}

Gascón S, Martínez-Jarreta B, González-Andrade JF, Santed Mlod Y, Rueda MA. Aggression towards health care workers in Spain: a multi-facility study to evaluate the distribution of growing violence among professionals, health facilities and departments. International Journal of Occupational and Environmental Health 2009;15(1):29-35.

\section{Gates 2011}

Gates DM, Gillespie G, Smith C, Rode J, Kowalenko T, Smith B. Using action research to plan a violence prevention program for emergency departments. Journal of Emergency Nursing 2011;37(1):32-9.

\section{Gerberich 2004}

Gerberich SG, Church TR, McGovern PM, Hansen HD, Nachreiner NM, Geisser MS, et al. An epidemiological study of the magnitude and consequences of work related violence: the Minnesota Nurses' Study. Occupational and Environmental Medicine 2004;61(6):495-503.

\section{Gerdtz 2013}

Gerdtz MF, Daniel C, Dearie V, Prematunga R, Bamert M, Duxbury $\mathrm{J}$. The outcome of a rapid training program on nurses' attitudes regarding the prevention of aggression in emergency departments: a multi-site evaluation. International Journal of Nursing Studies 2013;50(11):1434-45. [DOI: 10.1016/ j.ijnurstu.2013.01.007]

\section{GRADEPro 2014 [Computer program]}

McMaster University GRADEpro [Computer program on www.gradepro.org]. Hamilton, ON: McMaster University, 2014.

\section{Guay 2014}

Guay S, Goncalves J, Jarvis J. Verbal violence in the workplace according to victims' sex-a systematic review of the literature. Aggression and Violent Behaviour 2014;19(5):572-8. [DOI: 10.1016/j.avb.2014.08.001]

\section{Hahn 2008}

Hahn S, Zeller A, Needham I, Kok G, Dassen T, Halfens RJG. Patient and visitor violence in general hospitals: a systematic review of the literature. Aggression and Violent Behavior 2008;13(6):431-41.

\section{Hahn 2010}

Hahn S, Müller M, Needham I, Dassen T, Kok G, Halfens RJ. Factors associated with patient and visitor violence experienced by nurses in general hospitals in Switzerland: a cross-sectional survey. Journal of Clinical Nursing 2010;19(23-24):3535-46. [DOI: 10.1111/j.1365-2702.2010.03361.x]

\section{Hahn 2013}

Hahn S, Müller M, Hantikainen V, Kok G, Dassen T, Halfens RJ. Risk factors associated with patient and visitor violence in general hospitals: results of a multiple regression analysis. Journal of International Nursing Studies 2013;50(3):374-85. [DOI: 10.1016/j.ijnurstu.2012.09.018]

\section{Harrell 2011}

Harrell E. Workplace Violence, 1993-2009: National Crime Victimization Survey and the Census of Fatal Occupational Injuries. NCJ 233231. Washington: Bureau of Justice Statistics, 2011.

\section{Heckemann 2015}

Heckemann B, Zeller A, Hahn S, Dassen T, Schols JM, Halfens RJ. The effect of aggression management training programmes for nursing staff and students working in an acute hospital setting. A narrative review of current literature. Nurse Education Today 2015;35(1):212-9. [DOI: 10.1016/j.nedt.2014.08.003]

\section{Hegney 2006}

Hegney D, Eley R, Plank A, Buikstra E, Parker V. Workplace violence in Queensland, Australia: the results of a 
comparative study. International Journal of Nursing Practice 2006;12(4):220-31.

\section{Heponiemi 2014}

Heponiemi T, Kouvonen A, Virtanen M, Vänskä J, Elovainio M. The prospective effects of workplace violence on physicians' job satisfaction and turnover intentions: the buffering effect of job control. BMC Health Services Research 2014;14(19):1-8. [DOI: 10.1186/1472-6963-14-19]

\section{HEPRU 2003}

Health Policy and Economic Research Unit, British Medical Association. Violence at Work: The Experience of UK Doctors. London: British Medical Association, 2003.

\section{HEPRU 2008}

Health Policy and Economic Research Unit, British Medical Association. Violence in the Workplace: The Experience of Doctors in Great Britain. London: British Medical Association, 2008.

\section{Hershcovis 2010}

Hershcovis MS, Barling J. Toward a multi-focal approach to workplace aggression: a meta-analytic review of outcomes from different perpetrators. Journal of Organizational Behavior 2010;31(1):24-44.

\section{Higgins 2011}

Higgins JPT, Green S (editors). Cochrane Handbook for Systematic Reviews of Interventions Version 5.1.0 (updated March 2011). Cochrane, 2011. Available from www.cochranehandbook.org.

\section{Hills 2008}

Hills D. Relationships between aggression management training, perceived self-efficacy and rural general hospital nurses' experiences of patient aggression. Contemporary Nurse 2008;31(1):20-31.

\section{Hills 2012}

Hills D, Joyce C, Humphreys J. A national study of workplace aggression in Australian clinical medical practice. Medical Journal of Australia 2012;197(6):336-40.

\section{Hills 2013}

Hills D, Joyce C. A review of research on the prevalence, antecedents, consequences and prevention of workplace aggression in clinical medical practice. Aggression and Violent Behavior: A Review Journal 2013;18(5):554-69.

\section{Hills 2014}

Hills D, Joyce C. Workplace aggression in clinical medical practice: associations with job satisfaction, life satisfaction and self-rated health. Medical Journal of Australia 2014;201(9):535-40.

\section{Hills 2018}

Hills D, Lam L, Hills S. Workplace aggression experiences and responses of Victorian nurses, midwives and care personnel. Collegian 2018;25(6):575-82. [DOI: 10.1016/j.colegn.2018.09.003]

\section{Hinduja 2007}

Hinduja S. Workplace violence and negative affective responses: a test of Agnew's general strain theory. Journal of Criminal Justice 2007;35(6):657-66. [0047-2352]

\section{Hodgson 2004}

Hodgson MJ, Reed R, Craig T, Murphy F, Lehmann L, Belton L, et al. Violence in healthcare facilities: lessons from the Veterans Health Administration. Journal of Occupational \& Environmental Medicine 2004;46(11):1158-65.

\section{Hogh 2005a}

Hogh A, Henriksson M, Burr H. A 5-year follow-up study of aggression at work and psychological health. International Journal of Behavioral Medicine 2005;12(4):256-65. [1070-5503]

\section{Hogh 2005b}

Hogh A, Viitasara E. A systematic review of longitudinal studies of nonfatal workplace violence. European Journal of Work \& Organizational Psychology 2005;14(3):291-313. [1359432X]

\section{ILO 2002}

Joint Programme on Workplace Violence in the Health Sector. Framework Guidelines for Addressing Workplace Violence in the Health Sector. Geneva: International Labour Office, 2002.

\section{ILO 2003}

International Labour Organization Sectoral Activities Programme. Code of Practice on Workplace Violence in Services Sectors and Measures to Combat This Phenomenon. Geneva: International Labour Organization, 2003.

\section{Jansen 1997}

Jansen G, Dassen T, Moorer P. The perception of aggression. Scandinavian Journal of Caring Sciences 1997;11(1):51-5.

\section{Judy 2009}

Judy K, Veselik J. Workplace violence: a survey of paediatric residents. Occupational Medicine 2009;59(7):472-5.

\section{Kable 2012}

Kable A, Guest M, McLeod M. Resistance to care: contributing factors and associated behaviours in healthcare facilities. Journal of Advanced Nursing 2012;69(8):1747-60.

\section{Kansagra 2008}

Kansagra SM, Rao SR, Sullivan AF, Gordon JA, Magid DJ, Kaushal R, et al. A survey of workplace violence across 65 U.S. emergency departments. Academic Emergency Medicine 2008;15(12):1268-74. [1553-2712]

\section{Kvas 2014}

Kvas A, Seljak J. Unreported workplace violence in nursing. International Nursing Review 2014;61(3):344-51. [DOI: 10.1111/ inr.12106]

\section{Laker 2010}

Laker C, Gray R, Flach C. Case study evaluating the impact of de-escalation and physical intervention training. Journal of Psychiatric and Mental Health Nursing 2010;17(3):222-8. 


\section{Lanctôt 2014}

Lanctôt N, Guay S. The aftermath of workplace violence among healthcare workers: a systematic literature review of the consequences. Aggression and Violent Behavior 2014;19(5):492-501. [DOI: 10.1016/j.avb.2014.07.010]

\section{Lapierre 2005}

Lapierre LM, Spector PE, Leck JD. Sexual versus nonsexual workplace aggression and victims' overall job satisfaction: a meta-analysis. Journal of Occupational Health Psychology 2005;10(2):155-69.

\section{Laschinger 2014}

Laschinger HK. Impact of workplace mistreatment on patient safety risk and nurse-assessed patient outcomes. Journal of Nursing Administration 2014;44(5):284-90. [DOI: 10.1097/ NNA.0000000000000068]

\section{LeBlanc 2002}

LeBlanc MM, Kelloway EK. Predictors and outcomes of workplace violence and aggression. Journal of Applied Psychology 2002;87(3):444-53.

\section{LeBlanc 2006}

LeBlanc MM, Dupre KE, Barling J. Public-initiated violence. In: Kelloway EK, Barling J, Hurrell Jr JJ, editors(s). Handbook of Workplace Violence. Thousand Oaks: Sage Publications, Inc, 2006:261-80.

\section{Lewis 2005}

Lewis SE, Orford J. Women's experiences of workplace bullying: changes in social relationships. Journal of Community \& Applied Social Psychology 2005;15(1):29-47.

\section{Livingston 2010}

Livingston JD, Verdun-Jones S, Brink J, Lussier P, Nicholls T. A narrative review of the effectiveness of aggression management training programs for psychiatric hospital staff. Journal of Forensic Nursing 2010;6(1):15-28.

\section{Martínez-Jarreta 2007}

Martínez-Jarreta B, Gascón S, Santed MA, Goicoechea J. Medical-legal analysis of aggression towards health professionals. An approach to a silent reality and its consequences on health [Análisis médico-legal de las agresiones a profesionales sanitarios. Aproximación a una realidad silenciosa y a sus consecuencias para la salud]. Medicina Clínica 2007;128(8):307-10.

\section{Mayhew 2000}

Mayhew C. Preventing Client Initiated Violence: A Practical Handbook. Canberra: Australian Institute of Criminology, 2000.

\section{Mayhew 2001}

Mayhew C, Chappell D. Prevention of occupational violence in the health workforce. Working paper series 140. Taskforce on the Prevention and Management of Violence in the Health Workplace, Discussion Paper No. 2. Sydney: School of Industrial Relations and Organizational Behaviour and Industrial Relations Research Centre, University of New South Wales, 2001.

\section{Mayhew 2004}

Mayhew C. Occupational violence/bullying in the health industry. In: McCarthy P, Mayhew C, editors(s). Safeguarding the Organization Against Violence and Bullying: An International Perspective. Houndmills, UK: Oalgrave Macmillan, 2004:110-28.

\section{Mayhew 2007}

Mayhew C, Chappell D. Workplace violence: an overview of patterns of risk and the emotional/stress consequences on targets. International Journal of Law and Psychiatry 2007;30(4-5):327-39.

\section{McCarthy 2004}

McCarthy P. The safeguarding challenge. In: McCarthy P, Mayhew C, editors(s). Safeguarding the Organization Against Violence and Bullying: An International Perspective. Houndmills, UK: Oalgrave Macmillan, 2004:1-16.

\section{Nachreiner 2005}

Nachreiner NM, Gerberich SG, McGovern PM, Church TR, Hansen HE, Geisser MS, et al. Impact of training on work-related assault. Research in Nursing \& Health 2005;28(1):67-78.

\section{Needham 2004}

Needham I, Abderhalden C, Dassen T, Haug HJ, Fischer JE. The perception of aggression by nurses: psychometric scale testing and derivation of a short instrument. Journal of Psychiatric and Mental Health Nursing 2004;11(1):36-42.

\section{Needham 2005}

Needham I, Abderhalden C, Halfens RJ, Dassen T, Haug HJ, Fischer JE. The effect of a training course in aggression management on mental health nurses' perceptions of aggression: a cluster randomised controlled trial. International Journal of Nursing Studies 2005;42(6):649-55.

\section{Needham 2005a}

Needham I, Abderhalden C, Halfens RJG, Dassen T, Haug H-J, Fischer JE. The Impact of Patient Aggression on Carers Scale: instrument derivation and psychometric testing. Scandinavian Journal of Caring Sciences 2005;19(3):296-300.

\section{NICE 2006}

National Institute for Health and Clinical Excellence. Violence Clinical Practice Guidelines: The Short-Term Management of Disturbed/Violent Behaviour in In-Patient Psychiatric Settings and Emergency Departments. London: Royal College of Nursing, 2006.

\section{Niedhammer 2009}

Niedhammer I, David S, Degioanni S, Drummond A, Philip P. Workplace bullying and sleep disturbances: findings from a large scale cross-sectional survey in the French working population. Sleep 2009;32(9):1211-9.

\section{O'Brien-Pallas 2009}

O'Brien-Pallas L, Wang S, Hayes L, Laporte D. Creating work environments that are violence free. World Hospitals and Health Services 2009;45(2):12-8. 


\section{OSHA 2004}

Occupational Safety and Health Administration, US Department of Labor. Guidelines for Preventing Workplace Violence for Health Care \& Social Service Workers. Washington: US Department of Labor, 2004.

\section{Packham 2011}

Packham C. Violence at Work: Findings From the 2009/10 British Crime Survey. London: Health Service Executive, 2011.

\section{Paice 2009}

Paice E, Smith D. Bullying of trainee doctors is a patient safety issue. The Clinical Teacher 2009;6(1):13-7. [DOI: 10.1111/ j.1743-498X.2008.00251.x]

\section{Parent-Thirion 2007}

Parent-Thirion A, Marcias EF, Hurley J, Vermeylen G. Fourth European Working Conditions Survey. Dublin: European Foundation for the Improvement of Living and Working Conditions, 2007.

\section{Parker 2010}

Parker RM, Ceramidas DM, Forrest LE, Herath PM, McRae I. Patient initiated aggression and violence in the Australian general practice setting. Canberra: The Australian Primary Health Care Institute, 2010.

\section{Piquero 2013}

Piquero NL, Piquero AR, Craig JM, Clipper SJ. Assessing research on workplace violence, 2000-2012. Aggression and Violent Behavior 2013;18(3):383-94.

\section{Price 2015}

Price O, Baker J, Bee P, Lovell K. Learning and performance outcomes of mental health staff training in de-escalation techniques for the management of violence and aggression. British Journal of Psychiatry 2015;206(6):447-55. [DOI: 10.1192/ bjp.bp.114.144576]

\section{Ray 1994}

Ray EB, Miller KI. Social support, home/work stress, and burnout: Who can help? Journal of Applied Behavioral Science 1994;30(3):357-73.

\section{RevMan 5.3 [Computer program]}

The Nordic Cochrane Centre, The Cochrane Collaboration Review Manager (RevMan).. The Nordic Cochrane Centre, The Cochrane Collaboration, Version 5.3. Copenhagen: The Nordic Cochrane Centre, The Cochrane Collaboration, 2014.

\section{RevMan Web 2019 [Computer program]}

Review Manager Web. Cochrane, 2019. Available at revman.cochrane.org.

\section{Richter 2006}

Richter D, Needham I, Kunz S. The effects of aggression management training for mental health care and disability care staff: a systematic review. In: Richter D, Whittington $\mathrm{R}$, editors(s). Violence in Mental Health Settings. New York: Spring Science and Business Media, LLC, 2006:211-27.

\section{Roche 2010}

Roche M, Diers D, Duffield C, Catling-Paull C. Violence toward nurses, the work environment, and patient outcomes. Journal of Nursing Scholarship 2010;42(1):13-22.

\section{Rosenstein 2008}

Rosenstein AH, O'Daniel M. A survey of the impact of disruptive behaviors and communication defects on patient safety. Joint Commission Journal on Quality and Patient Safety 2008;34(8):464-71.

\section{Spector 2014}

Spector PE, Zhou ZE, Che XX. Nurse exposure to physical and nonphysical violence, bullying, and sexual harassment: a quantitative review. International Journal of Nursing Studies 2014;51:72-84.

\section{Spelten 2020}

Spelten E, Thomas B, O'Meara PF, Maguire BJ, FitzGerald D, Begg SJ. Organisational interventions for preventing and minimising aggression directed towards healthcare workers by patients and patient advocates.. Cochrane Database of Systematic Reviews 2020;4:E-pub. [DOI: DOI: 10.1002/14651858.CD012662.pub2.]

\section{Spencer 2018}

Spencer S, Johnson P, Smith I. De-escalation techniques for managing non-psychosis induced aggression in adults. Cochrane Database of Systematic Reviews 18 July 2018, Issue 7. Art. No: CD012034. [DOI: 10.1002/14651858.CD012034.pub2]

\section{Straus 1979}

Straus MA. Measuring intrafamily violence and conflict: the Conflict Tactics (CT) Scale. Journal of Marriage and Family 1979;41:75-88.

\section{Taylor 2010}

Taylor JL, Rew L. A systematic review of the literature: workplace violence in the emergency department. Journal of Clinical Nursing 2010;20:1072-85.

\section{Ventura-Madangeng 2009}

Ventura-Madangeng J, Wilson D. Workplace aggression experienced by registered nurses: a concept analysis. Nursing Praxis in New Zealand 2009;25(3):37-50.

\section{Viitasara 2002}

Viitasara E, Menckel E. Developing a framework for identifying individual and organizational risk factors for the prevention of violence in the health-care sector. Work 2002;19(2):117-23.

\section{Viitasara 2003}

Viitasara E, Sverke M, Menckel E. Multiple risk factors for violence to seven occupational groups in the Swedish caring sector. Industrial Relations 2003;58(2):202-31.

\section{Whittington 2002}

Whittington R, Higgins L. More than zero tolerance? Burnout and tolerance for patient aggression amongst mental health nurses in China and the UK. Acta Psychiatrica Scandinavica 2002;106:37-40. 


\section{Wieclaw 2006}

Wieclaw J, Agerbo E, Mortensen PB, Burr H, Tüchsen F, Bonde JP. Work related violence and threats and the risk of depression and stress disorders. Journal of Epidemiology and Community Health 2006;60(9):771-5.

\section{Wiskow 2003}

Wiskow C. Guidelines on Workplace Violence in the Health Sector. Comparison of Major Known National Guidelines and

\section{CHARACTERISTICS OF STUDIES}

Characteristics of included studies [ordered by study ID]
Strategies: United Kingdom, Australia, Sweden, USA (OSHA and California). Geneva: World Health Organization, 2003.

\section{Wyatt 1995}

Wyatt GE, Riederle M. The prevalence and context of sexual harassment among African American and White American women. Journal of Interpersonal Violence 1995;10(3):309-21.

* Indicates the major publication for the study

Anderson 2006

\title{
Study characteristics
}

Controlled before and after
Convenience sample: $\mathrm{n}=43$ (22 intervention, 21 control), group allocation not noted, missing data plan
not noted
Unit of measure: the healthcare worker
Study location/s
United States
Study setting/s
Full-service, small community hospital-99 beds-Joint Commission accredited
Withdrawals
Intervention participants who completed assessments but not the intervention were placed in a sepa-
rate group for data analysis

\section{Participants}

\author{
Health worker type/s \\ - Intervention participants: nurses (RNs, LVNs, nurse aides), security workers, home health workers, \\ nursing administration, nursing education, social work \\ - Control participants: rehab/physical therapy workers, laboratory workers, radiology workers, busi- \\ ness services workers
}

\section{Total number, number of health worker type subpopulations and proportions (\%)}

43 total: 22 intervention, 21 control

Appears that 10/22 intervention participants and 4/21 control participants were nurses. Proportions of subpopulations were not otherwise noted beyond separation by intervention/control groups, as noted above

\section{Mean age or age range}

21 to 60

\section{Gender}

35: 16/22 intervention female; 6/22 intervention male; 1 (control group) not reported

Workplace/s (e.g. mental health, emergency department) 
Full-service, 99-bed community hospital in United States (state/region not specified)

Work setting/s (e.g. hospital inpatient, hospital outpatient, community)

- Hospital inpatient and security (intervention participants). Additional intervention participants from home health, nursing administration, nursing education, and social work were included due to low participation

- Rehabilitation/physical therapy, laboratory, radiology, business services (control participants)

Work sector/s (e.g. public, private, non-government)

Private

\section{Inclusion and exclusion criteria}

Inclusion: hospital employees, not otherwise specified

Exclusion: not specified

Description of intervention and co-interventions (especially noting whether bundled with other organizational interventions)

Self-directed, 3-hour online training program consisting of 5 modules

Targeted knowledge, attitudes, and skills

- Risk assessment

- Theoretical models of aggression/violence

- De-escalation strategies

-Assertiveness training

-Physical contact skills/breakaway strategies

- Ethical and legal issues

- Debriefing procedures

\section{Comparison}

No training

Content of both intervention and control conditions, and co-interventions

As above

Pre- and post-intervention Workplace Violence Questionnaire and Demographics tool completed in contemporaneous windows by intervention and control groups

\section{Duration}

30-day period to complete pre-assessment, intervention, and post-assessment

\section{Intensity (e.g. frequency or levels of intervention)}

Single, 3-hour online training, to be undertaken at defined periods of time within a 30-day period (participants reported it took 45 to 180 minutes to complete)

\section{Number commencing}

22 intervention; 21 control 


\section{Number completing}

10 intervention; 13 control ( 5 additional intervention participants completed pre-assessments and post-assessments but did not complete online training)

\section{Adherence to protocol}

Noted with completion data as above. Participants not completing assessments were excluded from outcomes reporting

\section{Self-reported workplace violence events}

\section{Description of primary and secondary outcomes specified and collected}

- Self-reported workplace violence events (primary)-no differences between groups; significant change in pre-training and post-training WPV reports for training group

\section{Measurement instruments used and validation status (e.g. reported/not reported)}

Workplace Violence Questionnaire and Demographics tool. Reliability and validity reported

\section{Time points reported}

Pre-training (within 2 weeks); post-training (within 2 weeks)

\section{Controlling for biasing or confounding effects of co-interventions}

None reported. However, 5 intervention participants who completed pre-assessments and post-assessments but did not complete training intervention were assessed as a separate group from intervention and control groups

- Before training, 200 WPV events over a previous 6-month period were reported by 43 participants, with 155 being emotional-verbal, 38 sexual, and 6 physical (1 event was unclassified). Because of the small number of sexual and physical WPV reports, only the emotional-verbal WPV events were examined for pre-training and post-training results

-At 6 months post training, 65 WPV events were reported by the study's remaining 28 participants, which were re-grouped into Gp A (training, $n=29$ ), Gp B (no training, $n=13$ ), and Gp C (did not complete training, $n=5$ )

\section{Intervention (training)}

Baseline $(n=22)$

WPV events total $=135(67.5 \%)$

Type: emotional-verbal = 106 (78.5\%); sexual = 23 (17.5\%); physical = $5(4.0 \%)$

Post intervention $(n=10)$

WPV events total $=29(44.6 \%)$

Type: emotional-verbal $=21(72.5 \%) ;$ sexual $=8(27.5 \%) ;$ physical $=0(0 \%)$

\section{Control (no training)}

Baseline $(n=21)$

WPV events total $=65(32.5 \%)$

Type: emotional-verbal = 49 (75.5\%); sexual = 15 (23\%); physical = $1(1.5 \%)$

Post intervention Gp B (no training; $\mathrm{n}=13$ )

WPV events total $=21(32.4 \%)$ 
Type: emotional-verbal = 19 (90.4\%); sexual = $1(4.8 \%) ;$ physical $=1(4.8 \%)$

Post intervention Gp C (did not complete training; $n=5$ )

WPV events total $=15(23 \%)$

Type: emotional-verbal = 10 (67\%); sexual = $3(20 \%) ;$ physical = $2(13 \%)$

\section{Between-group differences}

- No statistically significant difference was found between Group A (completing training) and Group B or Group C (control participants/not completing training, respectively) for total WPV events

- Emotional-verbal WPV continued to be the most commonly reported (50 events) type of WPV and was statistically significant between Group B and Group C ( $M=-4.323 / F[2]=5.508 ; P=0.010)$

Statistical significance was also noted for pre-training and post-training reports of WPV for Group A (M = $2.100 / t[9]=2.272 ; P=0.049$ )

Time points at which primary and secondary outcomes were collected and categorization to short-term (6 months or less), medium-term (more than 6 months up to 12 months), and longterm (> 12 months) follow-up

6 months-short term

\section{Completion and attrition}

Only 10 of the intervention group completed the intervention, with 5 allocated to a third group (training not completed). Only 13 of the control group remained in the study

\section{Funding for study}

- Sigma Theta Tau International Honor Society of Nursing

- Southern Nursing Research Society

- Texas Health Resources

\section{Notable conflicts of interest of study authors}

None noted

\section{Contact author}

Cheryl Anderson; c.anderson@uta.edu

\section{Risk of bias}

\begin{tabular}{|c|c|c|}
\hline Bias & Authors' judgement & Support for judgement \\
\hline $\begin{array}{l}\text { Random sequence genera- } \\
\text { tion (selection bias) }\end{array}$ & High risk & $\begin{array}{l}\text { Participants were not randomized to intervention and control groups but were } \\
\text { designated }\end{array}$ \\
\hline $\begin{array}{l}\text { Allocation concealment } \\
\text { (selection bias) }\end{array}$ & High risk & $\begin{array}{l}\text { No allocation concealment-investigators enrolling participants could possi- } \\
\text { bly foresee assignments }\end{array}$ \\
\hline $\begin{array}{l}\text { Blinding of participants } \\
\text { and personnel (perfor- } \\
\text { mance bias) } \\
\text { All outcomes }\end{array}$ & Unclear risk & Insufficient information to permit judgment of "low risk" or "high risk" \\
\hline
\end{tabular}

$\begin{aligned} & \text { Blinding of outcome as- } \\ & \text { sessment (detection bias) }\end{aligned} \quad$ Unclear risk $\quad$ Insufficient information to permit judgment of "low risk" or "high risk"


Anderson 2006 (Continued)

All outcomes

Incomplete outcome data High risk Outcome data available for only 28 of the 43 participants
(attrition bias)

(attrition bias)

All outcomes

Selective reporting (re- Unclear risk $\quad$ Insufficient information to permit judgment of "low risk" or "high risk"
porting bias)

Other bias High risk Very small sample-likely underpowered and no randomization. No support
for external or internal validity

Arnetz 2000

\section{Study characteristics}

\section{Methods}

\section{Cluster-randomized controlled trial}

Convenience sample: 47 healthcare workplaces ( 65 workplaces invited), random assignment to intervention group ( 24 workplaces; $n=579$ at baseline) and control group ( 23 workplaces; $n=366$ at baseline)

Workplace randomization method not recorded

Randomization of participants at the level of the workplace (randomization by unit co-ordinator but method not recorded-likely convenience or targeted)

Unit of measure: the healthcare worker $(n=1500)$

Missing data reported, but treatment of missing data not discussed

\section{Study location/s}

Stockholm County, Sweden

\section{Study setting/s}

Emergency departments (5); geriatric care (7); psychiatric care (32); home health care (3)

\section{Withdrawals}

4 sites closed during the study period ( 3 intervention; 1 control)

\section{Participants}

\section{Health worker type/s}

Registered and practical nurses

Practical nurses with special training in mental health

Total number, number of health worker type subpopulations and proportions (\%)

$N=1500 ;$ subpopulations not reported

\section{Mean age or age range}

Not reported, but no significant differences were found by age

\section{Gender}

Not reported, but no significant differences were found by gender

\section{Workplace/s (e.g. mental health, emergency department)}


Arnetz 2000 (Continued)

Emergency department; geriatric care; psychiatric care; home health care

Work setting/s (e.g. hospital inpatient, hospital outpatient, community)

Emergency; inpatient; home health

Work sector/s (e.g. public, private, non-government)

Not explicitly noted

Inclusion and exclusion criteria

None noted

Description of intervention and co-interventions (especially noting whether bundled with other organizational interventions)

Intervention groups had regular individual feedback and follow-up group discussions of registered violent events (Violent Incident Form [VIF] used). Initial contact between project co-ordinator and worker reporting violent event (individual), ASAP following an event, followed by group discussion at a staff meeting ASAP after this. Group discussion structured to focus on the main points summarized on the VIF checklist

\section{Targeted knowledge, attitudes, and skills}

Who was aggressive? What was the course of events? What was the time, place, and nature of the incident? Did the victim sense in advance that something was going to happen? How was the situation handled? How did the victim react? Could the incident have been avoided or mitigated in any way?

\section{Comparison}

Registration of violent events (VIFs) without structured feedback and discussion

\section{Content of both intervention and control conditions, and co-interventions}

Registration of violent events via the VIF

\section{Duration}

Continuous for 12 months.

\section{Intensity (e.g. frequency or levels of intervention)}

Intervention after each registered violent event (1-on-1 with project co-ordinator) and in follow-up group discussions

\section{Number commencing}

24 sites commenced

\section{Number completing}

21 sites completed

\section{Adherence to protocol}

Not reported 
Secondary: awareness of violence prevention: significantly improved in intervention group

Measurement instruments used and validation status (e.g. reported/not reported)

Violent Incident Form

Face validity reported; reliability reported "good"

Time points reported

Baseline; 12 months

Controlling for biasing or confounding effects of co-interventions

None noted

\section{Intervention}

- The number of incidents registered by intervention work sites (total $=409$ ) ranged from 0 to 126 (mean $=17.0 ; \mathrm{SD}=31.7)$, with 5 intervention sites registering no incidents at all

- Intervention workplaces reported a total of 103 feedback discussion sessions (several violent incidents could be discussed at 1 session). The number of feedback sessions at any 1 workplace ranged from 0 to 56 (mean $=4.1 ; S D=11.7)$, with 15 workplaces reporting no feedback sessions at all

\section{Control}

The number of incidents registered by control workplaces (total $=271$ ) ranged from 0 to 91 incidents ( mean $=11.6 ; \mathrm{SD}=24.1$ ), with 9 workplaces reporting no incidents

\section{Between-group differences}

Follow-up questionnaire: staff perception of the IVF project

- Intervention and control groups differed significantly on 4 of the 15 additional questions concerning the individual's perception of the VIF project $(\mathrm{P}<0.05$ for all items)

- The intervention group reported that the project had given them better awareness of risk situations for violence toward staff ( $36 \%$ vs $29 \%$; $\mathrm{chi}^{2}=8.6 ; \mathrm{df}=3$ ); of how a potentially dangerous situation could be avoided or attenuated ( $34 \%$ vs $\left.26 \% ; \mathrm{chi}^{2}=5.0 ; \mathrm{df}=1\right)$; and of how nurses could best handle a patient or other person who became aggressive toward them in the workplace $\left(33 \%\right.$ vs $25 \%$; chi $\left.^{2}=10.4 ; \mathrm{df}=3\right)$

- Significantly more control group staff felt that the staff person who experiences a violent incident is helped by a general discussion of the event with other staff $\left(92 \%\right.$ vs $\left.87 \% ; X^{2}=4.2 ; \mathrm{df}=1\right)$

Risk factors for violence at work during the past year (pre-intervention and post-intervention) data-not included here

Time points at which primary and secondary outcomes were collected and categorization to short-term ( 6 months and less), medium-term (more than 6 months up to 12 months), and longterm (> 12 months) follow-up

Long-term: 12 months

Notes

Education and training for preventing and minimizing workplace aggression directed toward healthcare workers (Review) 
Arnetz 2000 (Continued)

None noted

\section{Risk of bias}

\begin{tabular}{|c|c|c|}
\hline Bias & Authors' judgement & Support for judgement \\
\hline $\begin{array}{l}\text { Random sequence genera- } \\
\text { tion (selection bias) }\end{array}$ & Unclear risk & $\begin{array}{l}\text { Insufficient information about the randomization process to permit judgment } \\
\text { of "low risk" or "high risk" }\end{array}$ \\
\hline $\begin{array}{l}\text { Allocation concealment } \\
\text { (selection bias) }\end{array}$ & Unclear risk & Insufficient information to permit judgment of "low risk" or "high risk" \\
\hline $\begin{array}{l}\text { Blinding of participants } \\
\text { and personnel (perfor- } \\
\text { mance bias) } \\
\text { All outcomes }\end{array}$ & Unclear risk & Insufficient information to permit judgment of "low risk" or "high risk" \\
\hline $\begin{array}{l}\text { Blinding of outcome as- } \\
\text { sessment (detection bias) } \\
\text { All outcomes }\end{array}$ & Unclear risk & Insufficient information to permit judgment of "low risk" or "high risk" \\
\hline $\begin{array}{l}\text { Incomplete outcome data } \\
\text { (attrition bias) } \\
\text { All outcomes }\end{array}$ & Low risk & $\begin{array}{l}\text { Differences in post-intervention response rates between intervention and con- } \\
\text { trol groups, but an adequate explanation provided for why attrition did not } \\
\text { bias outcomes }\end{array}$ \\
\hline $\begin{array}{l}\text { Selective reporting (re- } \\
\text { porting bias) }\end{array}$ & Low risk & Pre-specified measures were reported in sufficient detail \\
\hline Other bias & Low risk & No other bias determined \\
\hline
\end{tabular}

Fitzwater 2002

\section{Study characteristics}

\section{Methods}

\section{Cluster-randomized controlled trial}

Cluster sample: 2 nursing homes randomly selected and assigned as an intervention site (120 beds) and a comparison site (100 beds)

\section{Study location/s}

Metropolitan Midwestern United States, possibly Ohio, based on funding

\section{Study setting/s}

Two (2) randomly selected nursing homes, approximately the same size, randomly assigned as intervention (120 beds) and comparison sites (100 beds)

\section{Withdrawals}

None noted

Participants $\quad \mathbf{n}=\mathbf{2 0}$,

10 intervention, 10 control

\section{Health worker type/s}

Certified nursing assistants (CNAs) 
Total number, number of health worker type subpopulations and proportions (\%)

20 in total, 10 in each group; all CNAs

Mean age or age range

Range 25 to 55 years

Gender

$100 \%$ female

Workplace/s (e.g. mental health, emergency department)

Long-term care

Work setting/s (e.g. hospital inpatient, hospital outpatient, community)

Nursing home

Work sector/s (e.g. public, private, non-government)

Not stated

Inclusion and exclusion criteria

Inclusion: CNA, day shift, provide direct care for residents (e.g. bathing, feeding, dressing)

Exclusion: criteria not stated

Face-to-face program

4 hours of assault prevention education in two 2-hour sessions

Description of intervention and co-interventions (especially noting whether bundled with other organizational interventions)

4 hours of assault prevention education $(2 \times 2$-hour sessions)

Targeted knowledge, attitudes, and skills

Definitions of violence and assault, reasons for violent behavior, effective communication techniques, signs and symptoms of impending violence, ways to keep self and resident safe during a violent incident

\section{Comparison}

No assault prevention education

\section{Content of both intervention and control conditions, and co-interventions}

Complete assault log for 10 days pre-intervention and post-intervention (control group completed log in the same time period, but without completing educational intervention)

\section{Duration}

10-day period per participant pre-intervention and post-intervention, contemporaneously for intervention and control groups. Temporal spacing of 2 two-hour intervention sessions is not mentioned. Temporal spacing of logging periods relative to intervention period is not mentioned

\section{Intensity (e.g. frequency or levels of intervention)}

Intervention took place in two 2-hour sessions

\section{Number commencing}

20 (10 per group) 
Fitzwater 2002 (Continued)

\section{Number completing}

20 (10 per group) assumed; not explicitly stated

\section{Adherence to protocol}

Not explicitly stated, although stated that the 10 CNAs in the intervention group attended 4 hours of assault prevention education

Outcomes

Number of violent incidents before and after intervention (primary)

109 before education

54 after education

Significant differences in intervention group

Confidence in ability to prevent resident assaults; significant change in intervention group

Type of physical assault toward staff by patient

Physical injury to caregiver

Activity when assault occurred (context)

Measurement instruments used and validation status (e.g. reported/not reported)

Assault Log (noted to be previously validated, but not reported)

Instrument used to assess confidence not reported

\section{Time points reported}

2-week period pre-intervention, 2-week period post-intervention

\section{Controlling for biasing or confounding effects of co-interventions}

None noted, although reported percentage of participants who had previously received violence prevention training

- Most assaults occurred against CNAs during turning/transferring (33\%), dressing/changing, feeding, and bathing in both nursing homes (no other raw data provided)

\section{Intervention}

- Responsible for 10 to 24 residents during each day shift

- $90 \%$ of intervention home CNAs had received an injury from a resident at some point during their experience

- $90 \%$ of intervention home CNAs indicated they had been trained how to handle aggressive residents in both their previous and current nursing home jobs

- Average number of assaults reported for 2 weeks pre-intervention reduced from 13 to 6 for 2 weeks post-intervention $(P=0.02)$

- CNA confidence in ability to prevent resident assaults increased significantly $(P=0.005)$ from a mean of 3.5 (pre-intervention) to 4.4 (post-intervention)

\section{Control}

- Responsible for 8 to 16 residents during each day shift

- $60 \%$ of control home CNAs had received an injury from a resident

- $60 \%$ of control home CNAs indicated they had received previous training, and $50 \%$ reported current training related to aggressive residents 
- Average number of assaults reported for 2 weeks pre-intervention (3.4) remained similar to that reported for 2 weeks post-intervention (2.4)-no $\mathrm{P}$ value given

CNA confidence in ability to prevent resident assaults showed no significant change (no $P$ value given) from a mean of 3.4 (pre-intervention) to 3.3 (post-intervention)

Time points at which primary and secondary outcomes were collected and categorization to short-term ( 6 months and less), medium-term (more than 6 months up to 12 months), and longterm (> 12 months) follow-up

Short-term: follow-up took place for 2 weeks following the intervention. It is not explicitly stated whether this 2-week period occurred immediately following the intervention

Study authors and email address of corresponding author
Evelyn L. Fitzwater, fitzwael@ucmail.uc.edu
Donna M. Gates
College of Nursing at the University of Cincinnati in Ohio (no address provided)
Funding for study
Ohio Health Care Association Education Foundation

\section{Notable conflicts of interest of study authors}

None noted

\section{Risk of bias}

\begin{tabular}{lll}
\hline Bias & Authors' judgement & Support for judgement \\
\hline $\begin{array}{l}\text { Random sequence genera- } \\
\text { tion (selection bias) }\end{array}$ & High risk & $\begin{array}{l}\text { Two nursing homes, approximately the same size, were randomly selected } \\
\text { from a list of comparable nursing homes; then the } 2 \text { workplaces were "ran- } \\
\text { domly allocated" to intervention and control. Participants (10 in each group) } \\
\text { were selected by convenience sampling }\end{array}$ \\
\hline $\begin{array}{l}\text { Allocation concealment } \\
\text { (selection bias) }\end{array}$ & High risk & There was no allocation concealment \\
\hline $\begin{array}{l}\text { Blinding of participants } \\
\text { and personnel (perfor- } \\
\text { mance bias) } \\
\text { All outcomes }\end{array}$ & High risk & There was no blinding of participants or personnel \\
\hline
\end{tabular}

Blinding of outcome as- High risk There was no blinding of outcome assessment

Incomplete outcome data Unclear risk Insufficient information to permit judgment of "low risk" or "high risk"
(attrition bias)

All outcomes

\begin{tabular}{lll}
\hline $\begin{array}{l}\text { Selective reporting (re- } \\
\text { porting bias) }\end{array}$ & Unclear risk & Insufficient information to permit judgment of "low risk" or "high risk" \\
\hline Other bias & High risk & Very small sample (clusters and individual participants) -very underpowered \\
\hline
\end{tabular}


Irvine 2007

\section{Study characteristics}

Methods

\section{Randomized controlled trial}

Sample: $\mathrm{n}=72$ (34 intervention, 28 control)

Convenience sample from Internet-based and newspaper ad recruitment; randomized into treatment or control condition

Intervention (34), control (28)

Treatment of missing data not mentioned

\section{Study location/s}

USA. Web training and telephone interviews; no specific location

\section{Study setting/s}

Web training and telephone interviews

\section{Withdrawals}

72 participants qualified for the study and 62 completed both $T 1$ and $T 2$ assessments. No other information provided

\section{Participants}

\section{Health worker type/s}

Professional caregiver working with residents (e.g. NA, CNA)

Total number, number of health worker type subpopulations and proportions (\%)

62 nurse aids $(100 \%)$

\section{Mean age or age range}

No mean age or age range specified

\section{Gender}

Female $(53 ; 85.5 \%)$, male $(9 ; 14.5 \%)$

Female: treatment (30), control (23)

Workplace/s (e.g. mental health, emergency department)

Long-term care

Work setting/s (e.g. hospital inpatient, hospital outpatient, community)

Long-term care

Work sector/s (e.g. public, private, non-government)

Not specified

\section{Inclusion and exclusion criteria}

Eligibility:

- Individuals could verify that they worked with residents as professional caregivers (e.g. NAs, CNAs)

- They were no more than somewhat confident about handling aggressive situations on a 5-point Likert scale (i.e. not at all confident, not very confident, somewhat confident, very confident, or extremely confident) 
Irvine 2007 (Continued)

- They had scored 3 or less on a 5-point Likert scale asking about their training (i.e. none, very little, some, a moderate amount, a lot)

- They were required to enroll in the study from a computer that could play video over the Internet (i.e. Internet video-capable computer with broadband connection) and to have a valid email address

Interventions

Online training program

Description of intervention and co-interventions (especially noting whether bundled with other organizational interventions)

Interactive Internet training program including graphic images, video vignettes, testimonials

Not specified if bundled with organizational interventions

\section{Targeted knowledge, attitudes, and skills}

Skills for approaching an agitated resident exhibiting potentially dangerous behaviors and for safely de-escalating the situation

A.I.D. approach (Assess, Investigate, Do Something)

Person-centered care

\section{Comparison}

No Web training

\section{Content of both intervention and control condition, and co-interventions}

A total of 155 Web pages, 11 video vignettes, 16 narrator video clips, 71 voiceover clips, and 3 supportive NA video testimonials were produced into an interactive program that re-routed users to review content as part of the criterion-referenced instructional design

\section{Duration}

Intervention lasts 1 day

Intensity (e.g. frequency or levels of intervention)

One-time intervention

\section{Number commencing}

72

\section{Number completing}

62

\section{Adherence to protocol}

Not reported

Description of primary and secondary outcomes specified and collected

Primary outcome: effect of training on participants

- VST knowledge

- VST self-efficacy

- Attitude

- Self-efficacy

- Behavioral intentions 
Irvine 2007 (Continued)

\section{Secondary outcome:}

Satisfaction with Internet training

Telephone calls to 11 treatment group interviewees ranged from 20 to 30 minutes in length and occurred an average of 16.4 days $(S D=3.8)$ after use of the program

\section{Measurement instruments used and validation status (e.g. reported/not reported)}

No validation of the instrument was reported in this article

Assessment included a total of 44 items, administered pre-intervention and post-intervention. Post-test only, intervention group participants also responded to items assessing their satisfaction with the program and its design

- Nine items measured changes in participants' responses to 3 video depictions of aggressive situations (VSTs)-validation not reported

- The other 35 items measured changes in attitudes, self-efficacy, and behavioral intentions

- Finally, a convenience sample of 11 participants were interviewed by telephone after their submission of the T2 assessment

\section{Time points reported}

$\mathrm{T} 1$ = baseline assessment

$\mathrm{T} 2$ = 1 day after base assessment and intervention

Telephone interview $=16.4$ days average after $\mathrm{T} 2$ for 11 participants

Controlling for biasing or confounding effects of co-interventions

None reported

Satisfaction with program and website data-not included here

Time points at which primary and secondary outcomes were collected and categorization to short-term ( 6 months and less), medium-term (more than 6 months up to 12 months), and longterm (> 12 months) follow-up

Short-term

T2 was a post-test evaluation. A few participants accepted to take part in a telephone interview (average 16.4 days after $\mathrm{T} 2$ ) = short term

$\begin{array}{ll}\text { Outcomes } & \text { Main outcome measures: } \\ & \text { - Video situation testing (VST) knowledge } \\ & \text { VST self-efficacy } \\ & \text { - Attitudes } \\ \text { - Self-efficacy } \\ \text { - Behavioral intentions }\end{array}$

Blair Irvine, Michelle Bourgeois, Molly Billow, and John Seeley.

Corresponding author: Blair Irvine, birvine@orcasinc.com

\section{Funding for study}


Irvine 2007 (Continued)

National Institutes of Health (R43AG024675-01A1)

Notable conflicts of interest of study authors

Not reported

\section{Risk of bias}

\begin{tabular}{lll}
\hline Bias & Authors' judgement & Support for judgement \\
\hline $\begin{array}{l}\text { Random sequence genera- } \\
\text { tion (selection bias) }\end{array}$ & Unclear risk & $\begin{array}{l}\text { Insufficient information about the randomization process to permit judgment } \\
\text { of "low risk" or "high risk" }\end{array}$ \\
\hline $\begin{array}{l}\text { Allocation concealment } \\
\text { (selection bias) }\end{array}$ & Low risk & $\begin{array}{l}\text { Allocation concealment process sufficiently described to determine low risk of } \\
\text { selection bias }\end{array}$ \\
\hline $\begin{array}{l}\text { Blinding of participants } \\
\text { and personnel (perfor- } \\
\text { mance bias) } \\
\text { All outcomes }\end{array}$ & Low risk & Insufficient information to permit judgment of "low risk" or "high risk" \\
\hline
\end{tabular}

Blinding of outcome as-

High risk

Insufficient information to permit judgment of "low risk" or "high risk"

sessment (detection bias)

All outcomes

Incomplete outcome data $\quad$ Unclear risk $\quad$ Insufficient information to permit judgment of "low risk" or "high risk"
(attrition bias)

(attrition bias)

All outcomes

Selective reporting (re- $\quad$ Unclear risk $\quad$ Insufficient information to permit judgment of "low risk" or "high risk"
porting bias)

Selective reporting
porting bias)

Other bias High risk Trial is underpowered and provides no follow-up time after the intervention

Irvine 2012a

\section{Study characteristics}

Methods

\section{Randomized controlled trial}

Sample: $n=159$ (80 intervention, 79 control) with baseline, 1-month, and 2-month assessments: 159 nurse aides (NAs) or certified nursing assistants (CNAs), with 80 randomized to intervention, 79 to control

Despite low rates of missing data (0\% to 5\%), intention-to-treat analysis with maximum likelihood estimates used to impute missing data

Attrition was not significantly related to any measures, suggesting that dropping out of the study did not bias results

\section{Study location/s}

USA

\section{Study setting/s}

Online training

\section{Withdrawals}


151 (95\%) completing all 3 assessment questionnaires, 6 (4\%) 2 questionnaires, and 2 (1\%) 1 questionnaire

\section{Participants}

Online training program over 2 weeks

\section{Health worker type/s}

Direct caregiver in residential aged care (residents 50 years or older)

Total number, number of health worker type subpopulations and proportions (\%)

159 NAs or CNAs

\section{Mean age or age range}

No mean reported. Age range from 18 to 55 and up

\section{Gender}

Treatment: $87.5 \%$ (70) female/control: $86.1 \%(68)$ female

Workplace/s (e.g. mental health, emergency department)

Long-term residential care

Work setting/s (e.g. hospital inpatient, hospital outpatient, community)

Long-term residential care

Work sector/s (e.g. public, private, non-government)

Not specified

\section{Inclusion and exclusion criteria}

- Identification of employer for work as a direct caregiver (e.g. NA, certified nursing assistant [CNA]), for residents over 50 years of age)

- Self-rating of 0 to 3 on a 5 -point scale rating confidence to handle aggressive situations with residents (i.e. $0=$ not at all confident; $5=$ extremely confident)

- Self-reported level of aggression-specific training of 0 to 3 on a 5 -point scale (i.e. $0=$ none; $5=$ a lot)

- Able to enroll in the study from an Internet-video capable computer with broadband connection and to have a valid email address organizational interventions)

Intervention: $2 \times$ Internet training sessions, approximately 1 week apart

Targeted knowledge, attitudes, and skills

Knowledge of how to deal with aggressive behaviors

Attitudes, self-efficacy, and behavioral intentions regarding aggressive resident behaviors

\section{Comparison}

No training

\section{Content of both intervention and control conditions, and co-interventions}

Intervention: 
Irvine 2012a (Continued)

Visit 1: fundamental skills to safely de-escalate a situation with a resident exhibiting aggressive behavior; A.I.D. intervention strategy (Assess; Investigate; Do something); video vignettes

Visit 2: situation skills development concerning managing hits, hits with fists or arms; hair grabs and wrist grabs

Control:

No training

\section{Duration}

Study $=8$ weeks; Treatment $=4$ - week period

Intensity (e.g. frequency or levels of intervention)

2 sessions online, 1 week apart

Average amount of time spent on the program, across Visits 1 and 2, was 97.3 minutes (SD = $46.9 \mathrm{~min}$ )

Number commencing

159

\section{Number completing}

151

\section{Adherence to protocol}

Program usage and dose-response analysis:

At Visit 1, most treatment participants $(n=79 ; 98 \%)$ viewed both courses, and all participants saw at least 1 course. The average amount of time spent at Visit 1 was 65.8 minutes (SD $=32.1$ ). At Visit 2, most treatment participants $(n=68 ; 85 \%)$ viewed all 4 courses, $1(1 \%)$ viewed 3 courses, $2(3 \%)$ viewed 1 course, and $8(10 \%)$ did not view any course. The average amount of time spent at Visit 2 was 31.5 minutes $(S D=21.0)$

To assess dose-response change scores (defined as post-test measure minus pre-test measure), survey measures were correlated with total time of program use. Effect sizes in the small to medium range were found between time of program usage and improvement in self-efficacy $(r=.22 ; P=0.052)$ and empathy $(r=.22 ; \mathrm{P}=0.055)$

Time spent using the program was also correlated with post-intervention scores for VST self-efficacy ( $r$ $=.18 ; \mathrm{P}=0.138)$ and VST knowledge $(r=.49 ; \mathrm{P}<0.001)$, representing small and large effect sizes, respectively. Taken together, results suggest that treatment participants who invested more time using the program showed modest increases in study outcomes compared with those who used the program less

Outcomes

\section{Main outcome measures:}

- Video situation testing (VST) knowledge

-VST self-efficacy

- Attitudes

- Self-efficacy

- Empathy

- User acceptance

\section{Description of primary and secondary outcomes specified and collected}

No outcomes specified as primary or secondary. 
- Self-efficacy; medium to large effect; maintenance of effect

- Empathy; small effect

-Attitudes; medium to large effect; maintenance of effect

-Video situation test (VST) self-efficacy; medium effect size; maintenance of effect

-Video situation test (VST) knowledge; large effect size; maintenance of effect

- Greater gains for every measure for the treatment condition compared with the control condition

- Small to medium dose-response effect for self-efficacy and empathy, and small effect for improvement in attitudes. Dose response correlated with VST self-efficacy (small effect) and VST knowledge (large effect)

- Program acceptance: overall positive

\section{Measurement instruments used and validation status (e.g. reported/not reported)}

Investigator-constructed instrument. Psychometric testing (on non-knowledge questions) demonstrated excellent internal reliability and good test-retest reliability. Validity not reported

- Video situation testing (VST), which assessed participant reactions to video vignettes of resident behaviors (e.g. agitated resident swings a cane, resident grabs another resident forcefully) at T2 and T3

-VST knowledge-no reliability estimates indicated

-VST self-efficacy-excellent internal reliability $(a=.97)$ and good test-retest reliability in the control condition from T1 to T2 $(r=.63)$

- Self-efficacy-excellent internal reliability $(a=.93)$ and test-retest reliability in the control condition from $\mathrm{T} 1$ to $\mathrm{T} 2(\mathrm{r}=.76)$

-Attitudes-adequate internal reliability $(a=.67)$ and good test-retest reliability in the control condition from $\mathrm{T} 1$ to $\mathrm{T} 2(\mathrm{r}=.70)$

- Empathy-adequate internal reliability $(a=.62)$ and good test-retest reliability in the control condition from $\mathrm{T} 1$ to $\mathrm{T} 2(\mathrm{r}=.70)$

- User acceptance-not reported

\section{Time points reported}

$\mathrm{T} 1$ = baseline assessment

$\mathrm{T} 2$ = 4-week assessment

$\mathrm{T} 3=$ 8-week assessment

\section{Controlling for biasing or confounding effects of co-interventions}

No co-intervention reported

\section{Dose-response change scores}

To assess dose-response change scores (defined as post-test measure minus pre-test measure), survey measures were correlated with total time of program use. Effect sizes in the small to medium range were found between time of program usage and improvement in self-efficacy $(r=.22 ; P=0.052)$ and empathy $(r=.22 ; P=0.055)$, and a small effect was found for greater improvement in attitudes

\section{Satisfaction with program data-not included here}

Time points at which primary and secondary outcomes were collected and categorization to short-term ( 6 months and less), medium-term (more than 6 months up to 12 months), and longterm (> 12 months) follow-up 


\section{Study authors and email address of corresponding author}

A. Blair Irvine, Molly B. Billow, Donna M. Gates, Evelyn L. Fitzwater, John R. Seeley, Michelle Bourgeois

Corresponding author: A. Blair Irvine (birvine@orcasinc.com)

\section{Funding for study}

Grant from the National Institute on Aging to Oregon Center for Applied Science (R44AG024675)

Notable conflicts of interest of study authors

Not specified

\begin{tabular}{|c|c|c|}
\hline \multicolumn{3}{|l|}{ Risk of bias } \\
\hline Bias & Authors' judgement & Support for judgement \\
\hline $\begin{array}{l}\text { Random sequence genera- } \\
\text { tion (selection bias) }\end{array}$ & Unclear risk & Insufficient information to permit judgment of "low risk" or "high risk" \\
\hline $\begin{array}{l}\text { Allocation concealment } \\
\text { (selection bias) }\end{array}$ & Low risk & $\begin{array}{l}\text { Allocation concealment process sufficiently described to determine low risk of } \\
\text { selection bias }\end{array}$ \\
\hline $\begin{array}{l}\text { Blinding of participants } \\
\text { and personnel (perfor- } \\
\text { mance bias) } \\
\text { All outcomes }\end{array}$ & Low risk & Insufficient information to permit judgment of "low risk" or "high risk" \\
\hline $\begin{array}{l}\text { Blinding of outcome as- } \\
\text { sessment (detection bias) } \\
\text { All outcomes }\end{array}$ & High risk & Insufficient information to permit judgment of "low risk" or "high risk" \\
\hline $\begin{array}{l}\text { Incomplete outcome data } \\
\text { (attrition bias) } \\
\text { All outcomes }\end{array}$ & Low risk & $\begin{array}{l}\text { Effects of attrition (despite low numbers) tested and no differences detected } \\
\text { between those leaving and those remaining. Also, maximum likelihood esti- } \\
\text { mates used to impute missing values }\end{array}$ \\
\hline $\begin{array}{l}\text { Selective reporting (re- } \\
\text { porting bias) }\end{array}$ & Low risk & Pre-specified measures were reported in sufficient detail \\
\hline Other bias & Unclear risk & $\begin{array}{l}\text { Insufficient information to permit judgment of "low risk" or "high risk." Possi- } \\
\text { bly underpowered }\end{array}$ \\
\hline
\end{tabular}

Irvine 2012b

\section{Study characteristics}

Methods Cluster-randomized controlled trial (with cross-over)

6 long-term care (LTC) centers randomized into 3 immediate treatment $(I T ; n=58)$ and 3 delayed treatment (DT; $\mathrm{n}=45$ - control) conditions

Rates of missing data ranged from $0 \%$ to $21 \%$, and maximum likelihood estimates were used to impute missing values

\section{Study location/s}


Irvine 2012b (Continued)

Cincinnati, Ohio, USA

\section{Study setting/s}

Long-term care

\section{Withdrawals}

Of 103 study participants, 70 (69\%) completed all 3 assessment questionnaires, 17 (17\%) 2 questionnaires, and $15(15 \%) 1$ questionnaire

Participants

Health worker type/s

Nurse aides (NAs)

Total number, number of health worker type subpopulations and proportions (\%)

103 NAs (100\%)

\section{Mean age or age range}

No mean age or age range reported

\section{Gender}

Immediate treatment $(n=58): 77.6 \%$ female

Delayed treatment $(n=45): 97.8 \%$ female

Workplace/s (e.g. mental health, emergency department)

Long-term residential care facilities (LTCS)

Work setting/s (e.g. hospital inpatient, hospital outpatient, community)

Long-term residential care facilities

Work sector/s (e.g. public, private, non-government)

Not specified

Inclusion and exclusion criteria

Participants were required to be working at least 16 hours per week in a participating building

Description of intervention and co-interventions (especially noting whether bundled with other organizational interventions)

Intervention: 2 Internet training sessions, approximately 1 week apart

Targeted knowledge, attitudes, and skills

Knowledge of how to deal with aggressive behaviors

Attitudes, self-efficacy, and behavioral intentions regarding aggressive resident behaviours

\section{Comparison}

No training (in DT group)

Content of both intervention and control conditions, and co-interventions

Intervention: 
Irvine 2012b (Continued)

Visit 1: fundamental skills to safely de-escalate a situation with a resident exhibiting aggressive behavior; A.I.D. intervention strategy (Assess; Investigate; Do something); video vignettes

Visit 2: situation skills development concerning managing hits, hits with fists or arms; hair grabs and wrist grabs

\section{Duration}

Study: 16 weeks

Web training: over approximately 1 week

\section{Intensity (e.g. frequency or levels of intervention)}

Web training: 2 visits in approximately 1 week

\section{Number commencing}

103

\section{Number completing}

70 (69\%) completed all 3 assessment questionnaires, 17 (17\%) 2 questionnaires, and 15 (15\%) 1 questionnaire

\section{Adherence to protocol}

Not reported

Outcomes

Violence, measured as incidents per day (Table 2)

Mean $0.41(\mathrm{SD}=0.57)$ from 3 clusters IT, $\mathrm{n}=58$; and mean $0.41(\mathrm{SD}=0.57)$ from 3 clusters $\mathrm{DT}, \mathrm{n}=45$ control) conditions at $\mathrm{T} 2$

Main outcome measures:

- Video situation testing (VST) knowledge

- VST self-efficacy

- Attitudes

- Self-efficacy

- Empathy

- User acceptance

Description of primary and secondary outcomes specified and collected

No outcomes specified as primary or secondary

- Self-efficacy: no significant difference

- Empathy: no significant difference

- Attitudes: no significant difference

-Video situation test (VST) self-efficacy: no significant differences in treatment, but maintenance of effect in immediate treatment group

- Video situation test (VST) knowledge: significant increase with large effect size, and maintenance of effect in immediate treatment group

- Assault logs: significant decrease in incidents per day with maintenance effects in immediate treatment group (T1 to T3) 
- Small to medium dose-response effect for self-efficacy and empathy, and small effect for improvement in attitudes. Dose response correlated with VST self-efficacy (small effect) and VST knowledge (large effect)

- Program acceptance: overall positive

\section{Measurement instruments used and validation status (e.g. reported/not reported)}

Investigator-constructed instrument. Psychometric testing on some non-knowledge questions demonstrated good internal reliability and test-retest reliability. Validity not reported

- Self-efficacy-excellent internal reliability $(a=.9)$ and test-retest reliability in the DT condition from T1 to $\mathrm{T} 2(\mathrm{r}=.3)$

- Empathy-not reported

- Attitudes-not reported

-Video situation testing (VST), which assessed participant reactions to video vignettes of resident behaviors (e.g. agitated resident swings a cane, resident grabs another resident forcefully) at T2 and T3

-VST self-efficacy-excellent reliability $(a=.93)$ and good test-retest reliability in the IT group from T2 to $\mathrm{T} 3(r=.90)$

-VST knowledge-good test-retest reliability in the IT group from T2 to T3 ( $r=.74)$

-Assault logs-not relevant

- User acceptance-not reported

\section{Time points reported}

$\mathrm{T} 1=$ baseline assessment

$\mathrm{T} 2$ = 8-week assessment

$\mathrm{T} 3=16$-week assessment

Controlling for biasing or confounding effects of co-interventions

No co-intervention reported

Program acceptance (usability and impact) data-not included here

Time points at which primary and secondary outcomes were collected and categorization to short-term ( 6 months and less), medium-term (more than 6 months up to 12 months), and longterm (> 12 months) follow-up

Short-term

T2 (8 weeks) and T3 (16 weeks) = both short-term follow-ups

\section{Study authors and email address of corresponding author}

Blair Irvine (birvine@orcasinc.com), Mary B. Billow, Donna M. Gates, Evelyn L. Fitzwater, John Seeley, Michelle Bourgeois

Funding for study

National Institute on Aging (Grant No. R44AG024675)

Notable conflicts of interest of study authors

Not reported 
Irvine 2012b (Continued)

Risk of bias

\begin{tabular}{|c|c|c|}
\hline Bias & Authors' judgement & Support for judgement \\
\hline $\begin{array}{l}\text { Random sequence genera- } \\
\text { tion (selection bias) }\end{array}$ & Unclear risk & $\begin{array}{l}\text { Insufficient information about the randomization process to permit judgment } \\
\text { of "low risk" or "high risk" }\end{array}$ \\
\hline $\begin{array}{l}\text { Allocation concealment } \\
\text { (selection bias) }\end{array}$ & Unclear risk & $\begin{array}{l}\text { Insufficient information about the randomization process to permit judgment } \\
\text { of "low risk" or "high risk" }\end{array}$ \\
\hline $\begin{array}{l}\text { Blinding of participants } \\
\text { and personnel (perfor- } \\
\text { mance bias) } \\
\text { All outcomes }\end{array}$ & Unclear risk & $\begin{array}{l}\text { Insufficient information about the randomization process to permit judgment } \\
\text { of "low risk" or "high risk" }\end{array}$ \\
\hline $\begin{array}{l}\text { Blinding of outcome as- } \\
\text { sessment (detection bias) } \\
\text { All outcomes }\end{array}$ & Unclear risk & $\begin{array}{l}\text { Insufficient information about the randomization process to permit judgment } \\
\text { of "low risk" or "high risk" }\end{array}$ \\
\hline $\begin{array}{l}\text { Incomplete outcome data } \\
\text { (attrition bias) } \\
\text { All outcomes }\end{array}$ & Low risk & $\begin{array}{l}\text { Effects of attrition tested and no differences detected between those leaving } \\
\text { and those remaining }\end{array}$ \\
\hline $\begin{array}{l}\text { Selective reporting (re- } \\
\text { porting bias) }\end{array}$ & Unclear risk & $\begin{array}{l}\text { Insufficient information about the randomization process to permit judgment } \\
\text { of "low risk" or "high risk" }\end{array}$ \\
\hline Other bias & High risk & $\begin{array}{l}\text { Small samples of participants. Results reported for individual participants } \\
\text { rather than by cluster }\end{array}$ \\
\hline
\end{tabular}

Ming 2019

\section{Study characteristics}

\section{Methods}

\section{Randomized controlled trial}

392 participants from a Medical Center in Taipei. Participants were randomly assigned to intervention $(n=200)$ or control $(n=192)$ group. Participants in the control group received the intervention at the end of the study

\section{Study location/s}

Taipei

\section{Study setting/s}

Medical Center in Taipei

\section{Withdrawals}

2 people turned down the invitation to participate, and 6 participants completed the pre-test and did not complete the post-test

$\begin{array}{ll}\text { Participants } & \mathbf{n}=\mathbf{3 9 2} \\ & 200 \text { intervention, } 192 \text { control }\end{array}$

\section{Health worker type/s}

Nurses 


\section{Total number, number of health worker type subpopulations and proportions (\%)}

392 in total: 200 in the intervention group and 192 in the control group, all nurses

\section{Mean age or age range}

$34 \pm 9$ years

Gender

$94.5 \%$ female

Workplace/s (e.g. mental health, emergency department)

Medical Center in Taipei

Work setting/s (e.g. hospital inpatient, hospital outpatient, community)

Hospital

Work sector/s (e.g. public, private, non-government)

Not stated

\section{Inclusion and exclusion criteria}

Inclusion: to have been employed at medical center for 3 months or longer; no intention to resign in the past 6 months; willingness to participate

Exclusion: working in an area where there is a low incidence of workplace violence (obstetrics, operating room, gynecology, pediatrics, baby room, delivery room, supply center)

Interventions

Face-to-face program

Targeted knowledge, attitudes, and skills

Situational assessment and response, relevant regulations, communication skills, team support, emotional crisis resolution, verbal and physical violence prevention and treatment, self-defense and evasion skills

A 3-hour lesson over a single day dispensed by 5 healthcare/nursing teaching experts and 3 assistants

Description of intervention and co-interventions (especially noting whether bundled with other organizational interventions)

- Comprehensive discussion of WPV (60 minutes)

- Watch case video (10 minutes)

- Communication skills (30 minutes)

- Simulation (role-playing exercises) and discussion (50 minutes)

\section{Comparison}

No training. Training was, however, received by the control group following completion of data collection

\section{Content of both intervention and control conditions, and co-interventions}

As above. Control group received education and training after completion of the study

\section{Duration}

Not specified explicitly, but 3-hour training appears to have taken place over the course of a single day

\section{Intensity (e.g. frequency or levels of intervention)}

Intervention was a single 3-hour-long session 
Ming 2019 (Continued)

\author{
Number commencing \\ 400 recruited, 398 at start of intervention (2 declined to participate) \\ Number completing \\ 392 \\ Adherence to protocol
}

6 people did not complete the questionnaire in the post-test phase

\title{
Outcomes
}

\section{Time points reported}

T1: Participants completed the initial questionnaire before intervention, on September 1, 2016

T2: November 30, 2016 (i.e. 3 months later)

\section{Description of primary and secondary outcomes specified and collected}

No outcomes specified as primary or secondary.

- Awareness of workplace violence

- Attitudes toward workplace violence

- Self-efficacy in dealing with workplace violence

\section{Measurement instruments used and validation status (e.g. reported/not reported)}

Awareness of aggression was assessed with the short version of the Perception of Aggression Scale (POAS-S)-validated, KMO and Crohnbach's a reported

Atittude toward aggression was measured with the Management of Aggression and Violence Attitude Scale-validated, Crohnbach's a reported

Self-efficacy in dealing with aggression was measured using the Clinician Confidence in Coping With Patient Aggression Scale-validated, Crohnbach's a reported

\section{Controlling for biasing or confounding effects of co-interventions}

Not applicable

Time points at which primary and secondary outcomes were collected and categorization to short-term ( 6 months and less), medium-term (more than 6 months up to 12 months), and longterm (> 12 months) follow-up

Long-term: 12 months

Short-term: 3 months

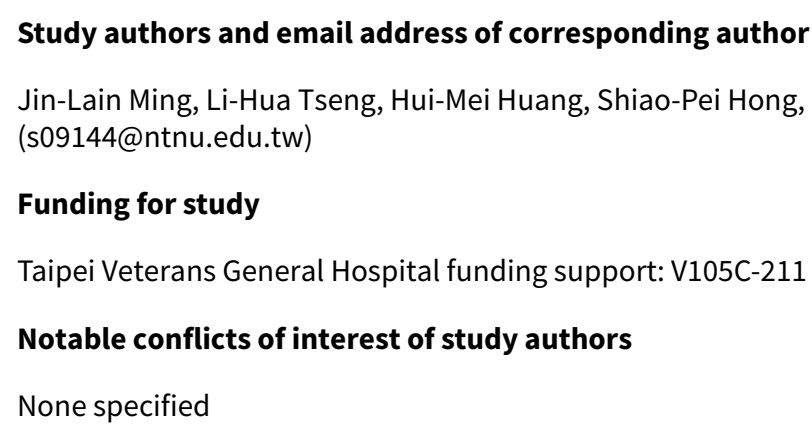


Ming 2019 (Continued)

\begin{tabular}{lll} 
Bias & Authors' judgement & Support for judgement \\
\hline $\begin{array}{ll}\text { Random sequence genera- } \\
\text { tion (selection bias) }\end{array}$ & Low risk & Random assignment of participants using a random numbers table \\
\hline
\end{tabular}

tion (selection bias)

Allocation concealment $\quad$ Unclear risk Insufficient information to permit judgment of "low risk" or "high risk"
(selection bias)

(selection bias)

Blinding of participants Unclear risk No blinding, but not deemed an issue
and personnel (perfor-
mance bias)
All outcomes

\begin{tabular}{|c|c|c|}
\hline $\begin{array}{l}\text { Blinding of outcome as- } \\
\text { sessment (detection bias) } \\
\text { All outcomes }\end{array}$ & Unclear risk & Insufficient information to assess risk of bias \\
\hline $\begin{array}{l}\text { Incomplete outcome data } \\
\text { (attrition bias) } \\
\text { All outcomes }\end{array}$ & Low risk & 392 of 400 participants completed the study \\
\hline $\begin{array}{l}\text { Selective reporting (re- } \\
\text { porting bias) }\end{array}$ & Low risk & All outcomes reported \\
\hline Other bias & Low risk & Sufficient sample size as determined by power analysis \\
\hline
\end{tabular}

Needham 2005

\section{Study characteristics}

Methods

\section{Cluster-randomized controlled trial}

Cluster sample: six psychiatric wards of 87 invited, with 3 wards randomly assigned as intervention and control sites. All 87 acute psychiatric wards in the German-speaking portion of Switzerland were invited to participate in the study. The 6 wards recruited for this study agreed to waiting list randomization. Three wards were randomly allocated to the intervention group and the remaining 3 to the control group. Treatment of missing data not reported

\section{Study location/s}

German-speaking portion of Switzerland

\section{Study setting/s}

6 acute psychiatric wards, with 3 randomly allocated to the intervention group and 3 to the control group

\section{Withdrawals}

None listed

\section{Health worker type/s}

Mental health nurses

Total number, number of health worker type subpopulations and proportions (\%) 


\section{Mean age or age range}

36.47 in intervention, 39.21 in control

\section{Gender}

18 female, 12 male in intervention; 14 female, 14 male in control

Workplace/s (e.g. mental health, emergency department)

Acute psychiatric wards

Work setting/s (e.g. hospital inpatient, hospital outpatient, community)

Not specified

Work sector/s (e.g. public, private, non-government)

Not specified

Inclusion and exclusion criteria

All 114 nurses working on the 6 participating wards were invited. No exclusion criteria were stated

$\mathbf{2 0} \times \mathbf{5 0}$-minute lessons over 5 consecutive days

Description of intervention and co-interventions (especially noting whether bundled with other organizational interventions)

20 lessons, each lasting 50 minutes, administered by trained psychiatric nurses on 5 consecutive days

\section{Targeted knowledge, attitudes, and skills}

Types and causes of aggression, the genesis of aggression, reflection on one's own aggressive components, theory on the various stages of aggressive incidents, behavior during aggressive situations, types of conflict management, communication and interaction, post-aggression procedures, workplace safety, prevention of aggression, breakaway techniques, and role-play

\section{Comparison}

No training. Training was, however, received by the control group after completion of the study

\section{Content of both intervention and control conditions, and co-interventions}

As above. The control group received education and training after completion of the study

\section{Duration}

5 consecutive days

\section{Intensity (e.g. frequency or levels of intervention)}

Intervention was $20 \times 50$-minute lessons

\section{Number commencing}

58: 30 in intervention wards, 28 in control wards

\section{Number completing}

Not specified

\section{Adherence to protocol}

Education and training for preventing and minimizing workplace aggression directed toward healthcare workers (Review) 


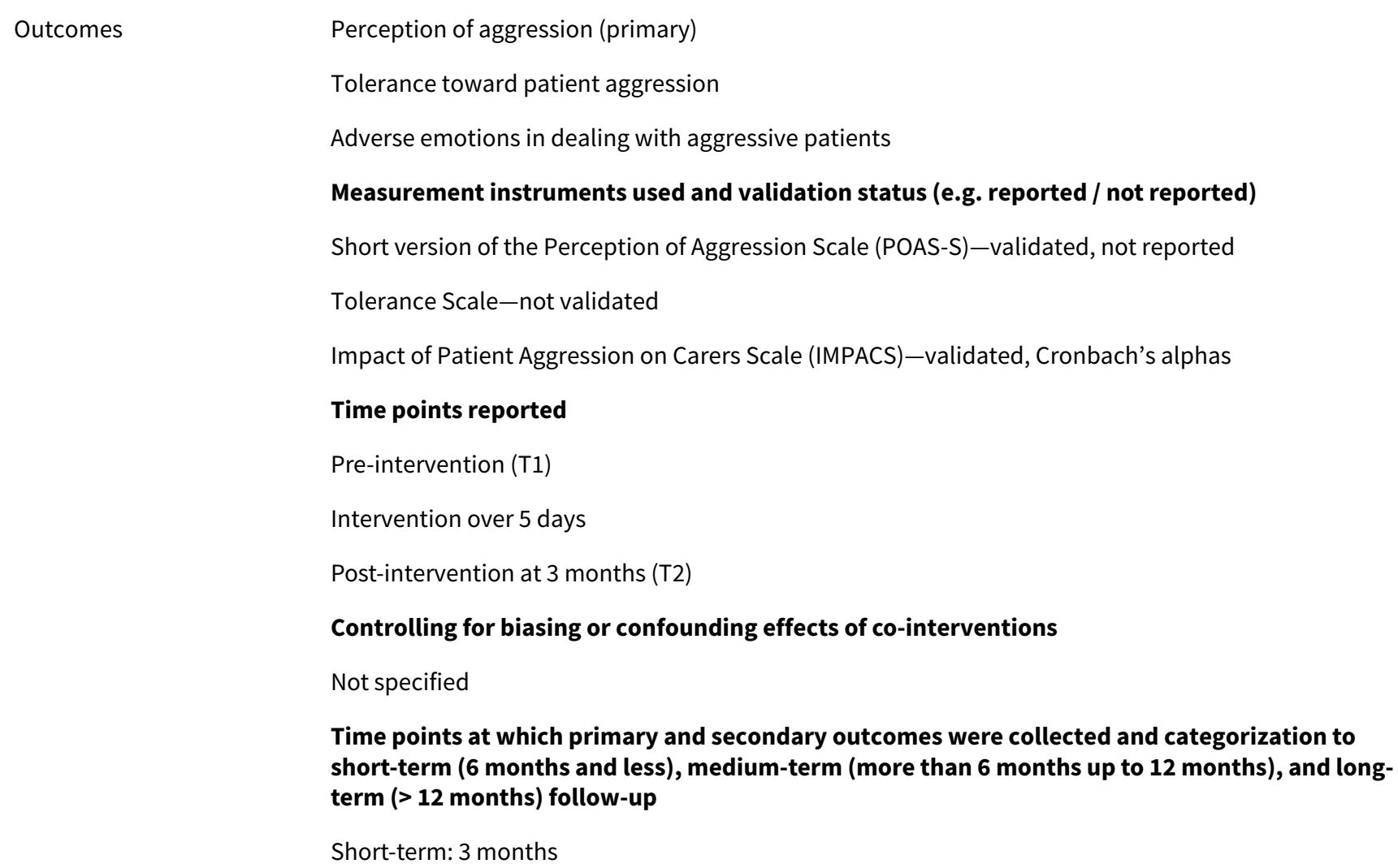

\begin{tabular}{lll}
\hline Bias & Authors' judgement & Support for judgement \\
\hline $\begin{array}{l}\text { Random sequence genera- } \\
\text { tion (selection bias) }\end{array}$ & Unclear risk & Insufficient information to permit judgment of "low risk" or "high risk" \\
\hline $\begin{array}{l}\text { Allocation concealment } \\
\text { (selection bias) }\end{array}$ & Unclear risk & Insufficient information to permit judgment of "low risk" or "high risk" \\
\hline $\begin{array}{l}\text { Blinding of participants } \\
\text { and personnel (perfor- } \\
\text { mance bias) } \\
\text { All outcomes }\end{array}$ & Unclear risk & Insufficient information to permit judgment of "low risk" or "high risk" \\
\hline
\end{tabular}

$\begin{array}{lll}\begin{array}{l}\text { Blinding of outcome as- } \\ \text { sessment (detection bias) }\end{array} & \text { Unclear risk } \quad \text { Insufficient information to permit judgment of "low risk" or "high risk" }\end{array}$


Needham 2005 (Continued)

All outcomes

\begin{tabular}{|c|c|c|}
\hline $\begin{array}{l}\text { Incomplete outcome data } \\
\text { (attrition bias) }\end{array}$ & High risk & $\begin{array}{l}\text { Poor response rates to outcome measure-no explanation for attrition nor ac- } \\
\text { counting for its impact provided }\end{array}$ \\
\hline
\end{tabular}

All outcomes

counting for its impact provided

\begin{tabular}{lll}
\hline $\begin{array}{l}\text { Selective reporting (re- } \\
\text { porting bias) }\end{array}$ & Low risk & Pre-specified measures were reported in sufficient detail \\
\hline Other bias & High risk & $\begin{array}{l}\text { Small samples of participants. Results reported for individual participants } \\
\text { rather than by cluster }\end{array}$ \\
\hline
\end{tabular}

Whittington 1996

\section{Study characteristics}

Methods

\section{Controlled before and after}

Non-random sample: $\mathrm{n}=155$ (47 intervention, 108 control), convenience sample (nominated to attend) from 155 nurses

\section{Study location/s}

London

\section{Study setting/s}

Two psychiatric hospitals-13 wards with the highest levels of violence across the 2 hospitals were selected for the study

\section{Withdrawals}

Not specified

\section{Participants}

\section{Health worker type/s}

Nurses

\section{Total number, number of health worker type subpopulations and proportions (\%)}

155 nurses. 47 (30.3\%) were attenders-chosen by ward managers. 108 (69.7\%) were non-attenders. $89 \%$ of attender group were qualified nurses compared with $71 \%$ of the control group

\section{Mean age or age range}

Not specified

\section{Gender}

Not specified. No significant sex differences between groups

Workplace/s (e.g. mental health, emergency department)

Mental health inpatients

Work setting/s (e.g. hospital inpatient, hospital outpatient, community)

Hospital inpatient

Work sector/s (e.g. public, private, non-government)

Not specified 
Whittington 1996 (Continued)

\section{Inclusion and exclusion criteria}

Not specified

One-day education session-1 part dealing with prevention of imminent violence, and the other dealing with the possible psychological consequences of assault

Description of intervention and co-interventions (especially noting whether bundled with other organizational interventions)

A 7-hour period of training in 1 day, divided into 4 sessions

Targeted knowledge, attitudes, and skills

- Risk assessment and diffusion techniques

- Post-assault management, legal issues, and the nature of traumatic stressful events

\section{Comparison}

Non-attenders of the sessions

\section{Content of both intervention and control conditions, and co-interventions}

Intervention: risk assessment and diffusion techniques, post-assault management, legal issues, and the nature of traumatic stressful events. Teaching methods included role-play of potentially violent situations and relaxation techniques

Control: no intervention

\section{Duration}

One-day sessions (held twice a week for 3 weeks; 6 groups in total)

Intensity (e.g. frequency or levels of intervention)

Intervention took place in 4 sessions over 7 hours in total

Number commencing

155 (47 attended the intervention; 108 were non-attenders)

Number completing

155

Adherence to protocol

Not stated

During the baseline period of 28 days before training, assaults on staff in both groups were counted. Following completion of the 6 training sessions, in the 28 days post intervention, assaults on staff were counted in the same way as before for both groups

Measurement instruments used and validation status (e.g. reported/not reported)

Not specified

Number of notified assaults on staff during a 28-day period immediately before training and during another 28-day period immediately after training 
Assaults were detected by contacting participating wards every weekday and asking all available staff about any notifiable assaults occurring in the preceding 24 to 48 hours. No validation

\section{Time points reported}

28 days pre-intervention, 28 days post-intervention

\section{Controlling for biasing or confounding effects of co-interventions}

Not specified

Mention made of individual effect and willingness of certain staff to take part in any training that is offered. Post-data collection analysis showed a higher number of assaults on the attenders group pre-intervention

Overall, in the study wards, reported violence fell by $31 \%$ after training, with 58 assaults reported in the month before training and 40 in the month after training

\section{Intervention}

Reported assaults for attenders fell from 22 (pre-intervention) to 19 (post-intervention) - not statistically significant (McNemar's test)

\section{Control}

Reported assaults for non-attenders fell from 13 (pre-intervention) to 8 (post-intervention) -not statistically significant (McNemar's test)

\section{Other comparisons}

For high-compliance wards ( $\geq 50 \%$ of staff trained), reported assaults fell from 40 (pre-intervention) to 12 (post-intervention)

For low-compliance wards ( $<50 \%$ of staff trained), reported assaults increased from 18 (pre-intervention) to 28 (post-intervention)

This difference between wards according to compliance was statistically significant $\left(r^{2}=12.9 ; 1 \mathrm{df} ; \mathrm{P}<\right.$ 0.05) using the $\mathrm{Chi}^{2}$ test of association

Time points at which primary and secondary outcomes were collected and categorization to short-term ( 6 months and less), medium-term (more than 6 months up to 12 months), and longterm (> 12 months) follow-up

28 days post intervention-short-term

\section{Notes}

\section{Funding for the study}

Not specified

\section{Notable conflicts of interest of study authors}

Not specified

NOTE: intervention group worked in the same settings as control group (assault rates for participants were the main outcome measure)

\section{Risk of bias}

\begin{tabular}{lll}
\hline Bias & Authors' judgement & Support for judgement \\
\hline $\begin{array}{l}\text { Random sequence genera- } \\
\text { tion (selection bias) }\end{array}$ & High risk & $\begin{array}{l}\text { No randomization into intervention or control groups-participants were nom- } \\
\text { inated by ward managers }\end{array}$ \\
\hline
\end{tabular}


Whittington 1996 (Continued)

\begin{tabular}{l}
$\begin{array}{l}\text { Allocation concealment } \\
\text { (selection bias) }\end{array}$ \\
\hline
\end{tabular}

Blinding of participants

High risk

No blinding. Participants knew that they were sent to a course about coping and personnel (perforwith violent persons, and the control group worked on the wards while intermance bias) vention group members were away on the course

All outcomes

Blinding of outcome as- High risk Insufficient information to permit judgment of "low risk" or "high risk"
sessment (detection bias)

All outcomes

\begin{tabular}{lll}
\hline $\begin{array}{l}\text { Incomplete outcome data } \\
\text { (attrition bias) } \\
\text { All outcomes }\end{array}$ & Low risk & No attrition or other non-engagement in the intervention apparent \\
\hline $\begin{array}{l}\text { Selective reporting (re- } \\
\text { porting bias) }\end{array}$ & Unclear risk & Insufficient information to permit judgment of "low risk" or "high risk" \\
\hline Other bias & High risk & $\begin{array}{l}\text { Small sample size, groups not matched, no support for external or internal va- } \\
\text { lidity }\end{array}$
\end{tabular}

CNA: certified nursing assistant.

DT: delayed treatment.

IMPACS: I.mpact of Patient Aggression on Carers Scale

KMO: Kaiser-Meyer-Olkin test.

LTC: long-term care.

LVN: licensed vocational nurse.

NA: nursing assistant.

POAS-S: Perception of Aggression Scale.

$\mathrm{RN}$ : registered nurse.

SD: standard deviation.

VIF: violent incident form.

VST: video situation test.

WPV: workplace violent incident.

Characteristics of excluded studies [ordered by study ID]

\begin{tabular}{ll}
\hline Study & Reason for exclusion \\
\hline Adams 2017 & No comparison group \\
\hline Beech 2003 & No control group \\
\hline Beech 2008 & No control group \\
\hline Cailhol 2007 & No control group \\
\hline Casalino 2015 & No control group \\
\hline Cooper 2006 & Study participants were patients-not healthcare workers \\
\hline Fernandes 2002 & No control group \\
\hline Gates 2013 & $\begin{array}{l}\text { Grant application for multi-program intervention. Results reported separately (see Gillespie 2013, } \\
\text { Gillespie 2014, Kowalenko 2014) }\end{array}$ \\
\hline \hline
\end{tabular}

Education and training for preventing and minimizing workplace aggression directed toward healthcare workers (Review) 


\begin{tabular}{|c|c|}
\hline Study & Reason for exclusion \\
\hline Gerdtz 2012 & No control group \\
\hline Gertz 1980 & No control group \\
\hline Gillespie 2013 & No control group in evaluation of multi-intervention program \\
\hline Gillespie 2014 & Education intervention applied to intervention and control groups \\
\hline Ishak 2002 & No pre-intervention or post-intervention control group measures \\
\hline Kang 2017 & Intervention aimed at preventing aggression between workers \\
\hline Kim 2018 & Did not evaluate an intervention \\
\hline Kowalenko 2014 & $\begin{array}{l}\text { Conference abstract reporting outcomes of a multi-intervention program (see Gillespie 2013, Gille- } \\
\text { spie 2014) }\end{array}$ \\
\hline Li 2018 & Did not evaluate an intervention \\
\hline Lipscomb 2004a & No control group \\
\hline Lipscomb 2004b & Multi-intervention program reported with no specific findings related to education alone \\
\hline Lipscomb 2006 & Multi-intervention program reported with no specific findings related to education alone \\
\hline McElaney 2008 & Did not report on an intervention for healthcare workers \\
\hline McIntosh 2003 & Outcomes not relevant \\
\hline Meehan 2006 & No control group \\
\hline Ore 2002 & No concurrent control group. "Controls" selected post intervention \\
\hline Peek-Asa 2002 & Cross-sectional surveys before and after legislation enactment \\
\hline Rittenmeyer 2013 & Literature review \\
\hline Shah 1998 & No control group \\
\hline Vousden 1987 & A descriptive report-not an intervention study \\
\hline
\end{tabular}

\section{DATA AND ANALYSES}

\section{Comparison 1. Number of episodes of aggression}

\begin{tabular}{lllll}
\hline Outcome or subgroup title & No. of studies & $\begin{array}{l}\text { No. of partici- } \\
\text { pants }\end{array}$ & Statistical method & Effect size \\
\hline 1.1 CRCT short-term follow-up & 2 & 49 & $\begin{array}{l}\text { Std. Mean Difference (IV, Random, } \\
95 \% \mathrm{Cl})\end{array}$ & $-0.33[-1.27,0.61]$ \\
\hline
\end{tabular}




\begin{tabular}{|c|c|c|c|c|}
\hline Outcome or subgroup title & No. of studies & $\begin{array}{l}\text { No. of partici- } \\
\text { pants }\end{array}$ & Statistical method & Effect size \\
\hline $\begin{array}{l}\text { 1.1.1 Short duration-face-to- } \\
\text { face }\end{array}$ & 1 & 10 & $\begin{array}{l}\text { Std. Mean Difference (IV, Random, } \\
95 \% \mathrm{Cl} \text { ) }\end{array}$ & $-1.03[-2.40,0.34]$ \\
\hline 1.1.2 Long duration-online & 1 & 39 & $\begin{array}{l}\text { Std. Mean Difference (IV, Random, } \\
95 \% \mathrm{CI} \text { ) }\end{array}$ & $0.00[-0.63,0.63]$ \\
\hline 1.2 CBA short-term follow-up & 1 & 155 & Risk Ratio (IV, Random, 95\% CI) & $2.30[0.97,5.42]$ \\
\hline $\begin{array}{l}\text { 1.2.1 Short duration-face-to- } \\
\text { face }\end{array}$ & 1 & 155 & Risk Ratio (IV, Random, 95\% CI) & $2.30[0.97,5.42]$ \\
\hline 1.3 CBA short-term follow-up & 1 & 23 & $\begin{array}{l}\text { Std. Mean Difference (IV, Random, } \\
95 \% \mathrm{CI} \text { ) }\end{array}$ & $-1.24[-2.16,-0.33]$ \\
\hline 1.4 CRCT long-term follow-up & 1 & 291 & Risk Ratio (IV, Random, 95\% CI) & $1.14[0.95,1.37]$ \\
\hline $\begin{array}{l}\text { 1.4.1 Extended duration-face- } \\
\text { to-face }\end{array}$ & 1 & 291 & Risk Ratio (IV, Random, 95\% CI) & $1.14[0.95,1.37]$ \\
\hline
\end{tabular}

\section{Analysis 1.1. Comparison 1: Number of episodes of aggression, Outcome 1: CRCT short-term follow-up}

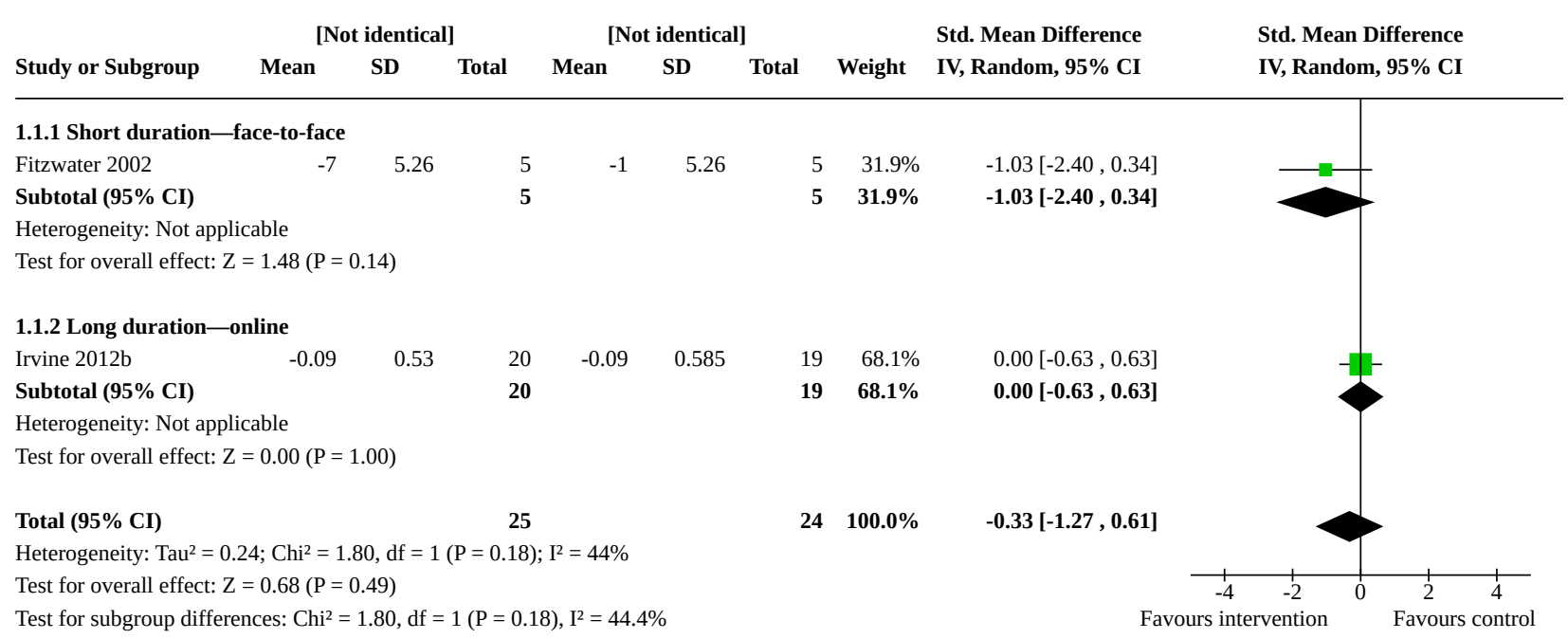


Analysis 1.2. Comparison 1: Number of episodes of aggression, Outcome 2: CBA short-term follow-up

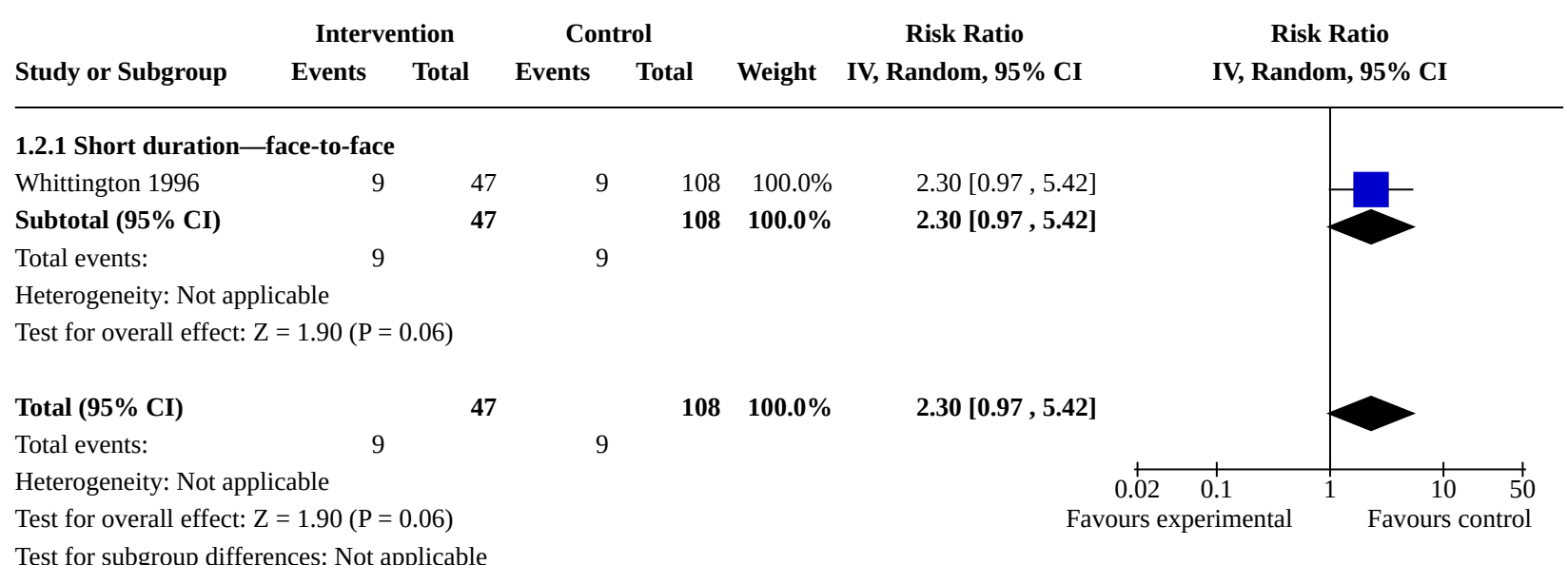

\section{Analysis 1.3. Comparison 1: Number of episodes of aggression, Outcome 3: CBA short-term follow-up}

\begin{tabular}{lcccccccc} 
& \multicolumn{3}{c}{ Experimental } & \multicolumn{3}{c}{ Control } & & \multicolumn{2}{c}{ Std. Mean Difference } & $\begin{array}{l}\text { Std. Mean Difference } \\
\text { Study or Subgroup }\end{array}$ \\
\hline
\end{tabular}

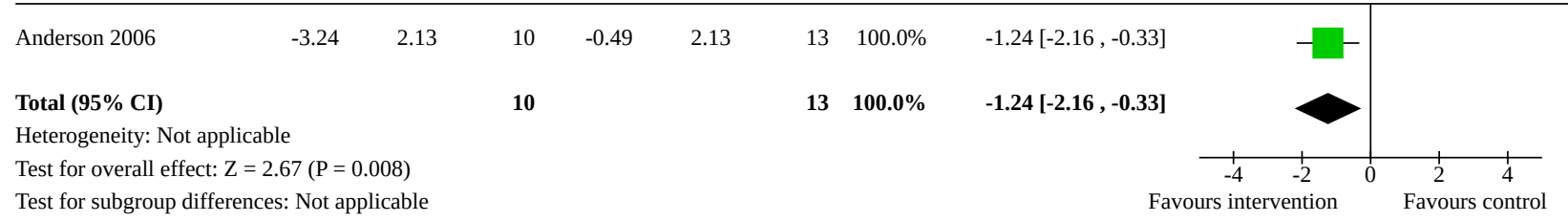

\section{Analysis 1.4. Comparison 1: Number of episodes of aggression, Outcome 4: CRCT long-term follow-up}

\begin{tabular}{|c|c|c|c|c|c|c|c|}
\hline \multirow[b]{2}{*}{ Study or Subgroup } & \multicolumn{2}{|c|}{ Intervention } & \multicolumn{2}{|c|}{ Control } & \multicolumn{2}{|r|}{ Risk Ratio } & \multirow{2}{*}{$\begin{array}{c}\text { Risk Ratio } \\
\text { IV, Random, 95\% CI }\end{array}$} \\
\hline & Events & Total & Events & Total & Weight & IV, Random, 95\% CI & \\
\hline
\end{tabular}

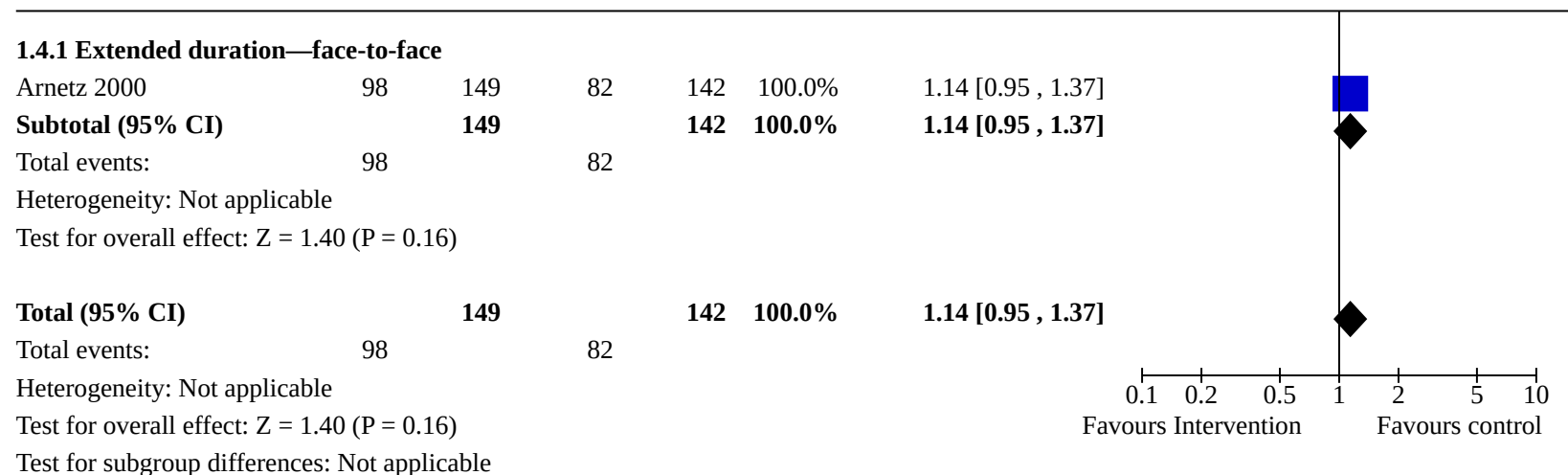


Comparison 2. Personal knowledge about aggression

\begin{tabular}{lllll}
\hline Outcome or subgroup title & No. of studies & $\begin{array}{l}\text { No. of partici- } \\
\text { pants }\end{array}$ & Statistical method & Effect size \\
\hline $\begin{array}{l}\text { 2.1 Knowledge about aggression (RCT/ } \\
\text { CRCT) - short-term follow-up }\end{array}$ & 1 & 62 & $\begin{array}{l}\text { Std. Mean Difference (IV, Ran- } \\
\text { dom, 95\% Cl) }\end{array}$ & 0.86 [0.34, 1.38] \\
\hline $\begin{array}{lllll}2.1 .1 \text { Self-paced duration-online } \\
\text { 2.2 Knowledge about aggression (RCT/ }\end{array}$ & 1 & 62 & $\begin{array}{l}\text { Std. Mean Difference (IV, Ran- } \\
\text { dom, 95\% Cl) }\end{array}$ & 0.86 [0.34, 1.38] \\
\begin{tabular}{l} 
CRCT)-long-term follow-up \\
\hline
\end{tabular} & 1 & 291 & $\begin{array}{l}\text { Risk Ratio (M-H, Fixed, 95\% } \\
\text { Cl) }\end{array}$ & 1.26 [0.90, 1.75] \\
\hline
\end{tabular}

Analysis 2.1. Comparison 2: Personal knowledge about aggression, Outcome 1: Knowledge about aggression (RCT/CRCT)-short-term follow-up

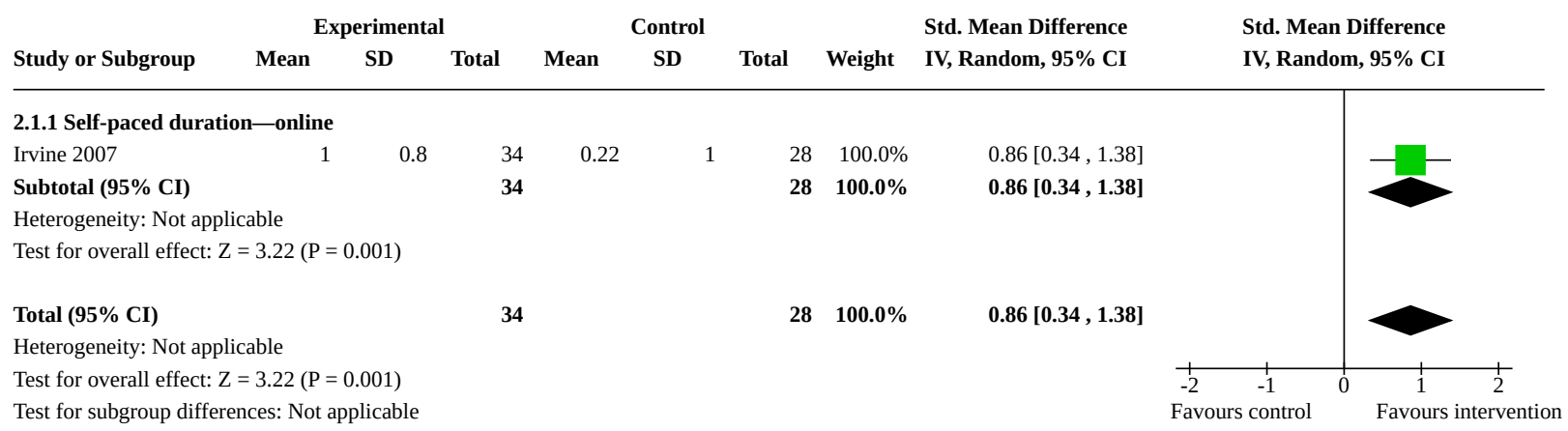

Analysis 2.2. Comparison 2: Personal knowledge about aggression, Outcome 2: Knowledge about aggression (RCT/CRCT)-long-term follow-up

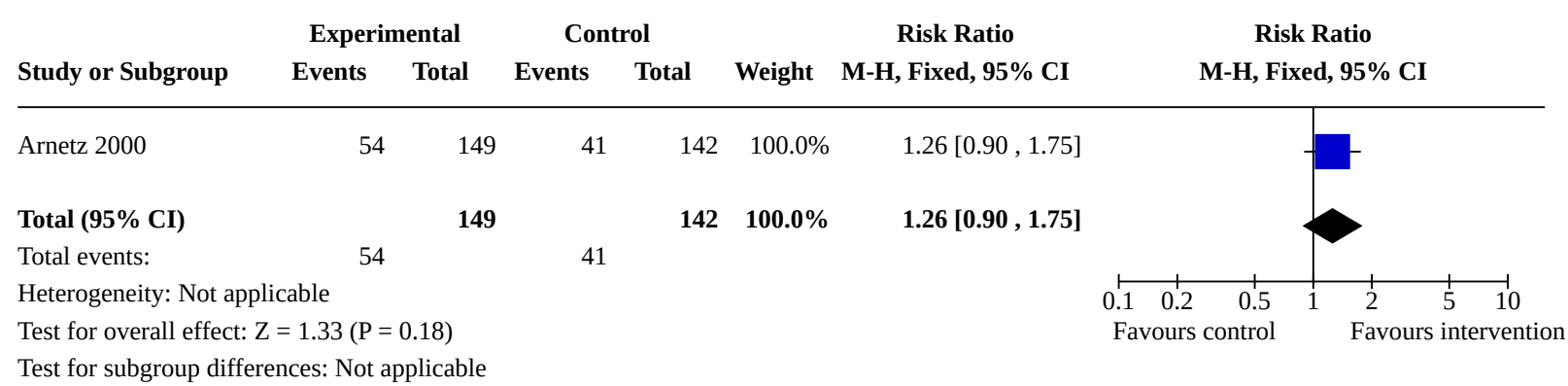

\section{Comparison 3. Attitudes}

\begin{tabular}{llll}
\hline Outcome or subgroup title & No. of studies & $\begin{array}{l}\text { No. of partici- } \\
\text { pants }\end{array}$ & Statistical method \\
\hline $\begin{array}{l}\text { 3.1 Attitudes (RCT/CRCT)- } \\
\text { short-term follow-up }\end{array}$ & 5 & 683 & $\begin{array}{l}\text { Std. Mean Difference (IV, Random, } \\
95 \% \text { CI) }\end{array}$ \\
\hline
\end{tabular}




\begin{tabular}{lllll}
\hline Outcome or subgroup title & No. of studies & $\begin{array}{l}\text { No. of partici- } \\
\text { pants }\end{array}$ & Statistical method & Effect size \\
\hline $\begin{array}{llll}\text { 3.1.1 Short duration-face-to- } \\
\text { face }\end{array}$ & 1 & 392 & $\begin{array}{l}\text { Std. Mean Difference (IV, Random, } \\
95 \% \text { Cl) }\end{array}$ & 0.78 [0.58, 0.99] \\
\hline $\begin{array}{llll}\text { 3.1.2 Self-paced-online } \\
\text { 3.1.3 Long duration-online }\end{array}$ & 1 & 62 & $\begin{array}{l}\text { Std. Mean Difference (IV, Random, } \\
95 \% \text { Cl) }\end{array}$ & 1.23 [0.69, 1.78] \\
\hline $\begin{array}{l}\text { 3.1.4 Long duration-face-to- } \\
\text { face }\end{array}$ & 1 & 198 & $\begin{array}{l}\text { Std. Mean Difference (IV, Random, } \\
95 \% \text { Cl) }\end{array}$ & 0.33 [0.05, 0.61] \\
\hline
\end{tabular}

\section{Analysis 3.1. Comparison 3: Attitudes, Outcome 1: Attitudes (RCT/CRCT)-short-term follow-up}

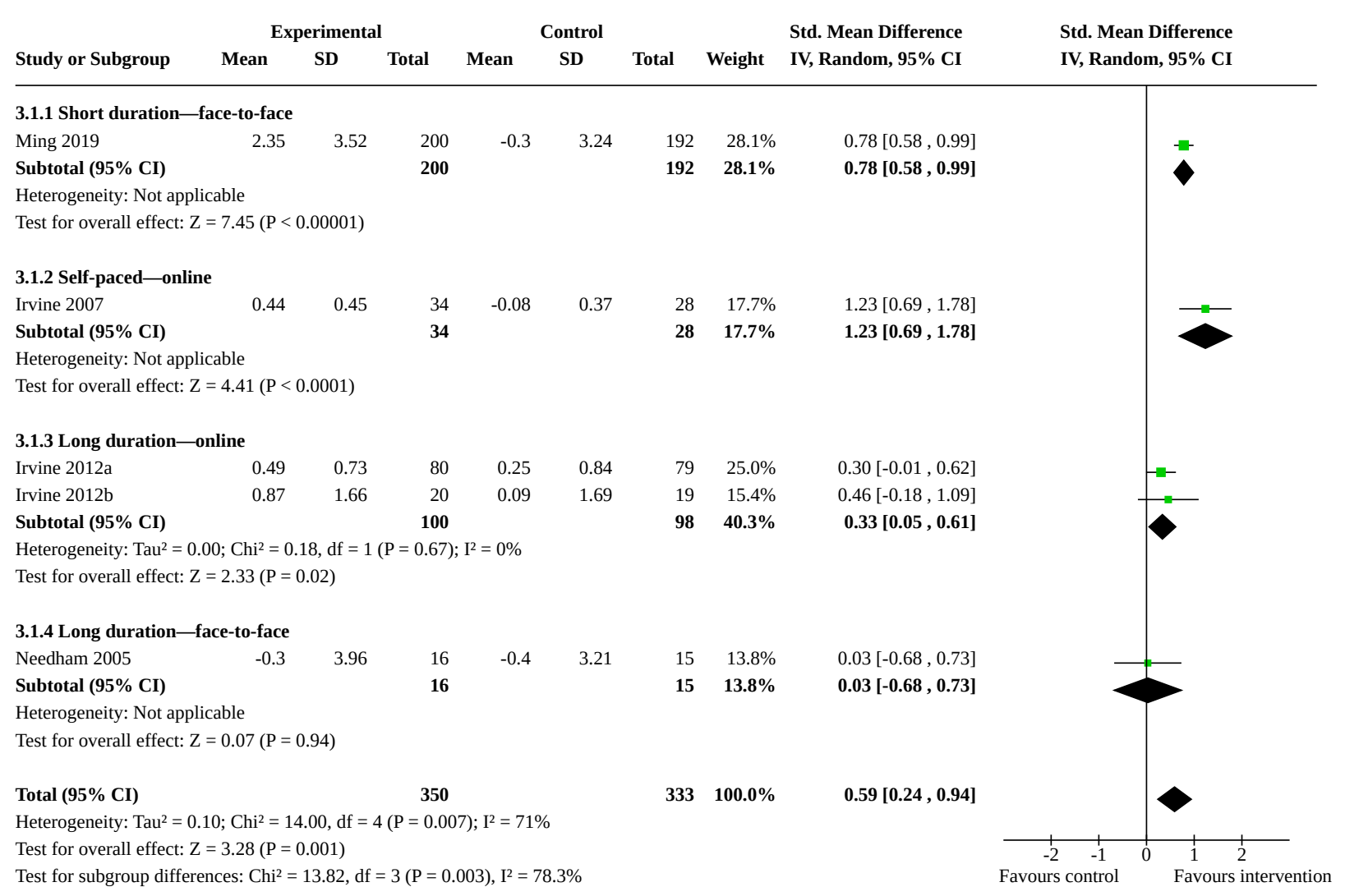

\section{Comparison 4. Skills}

\begin{tabular}{lllll}
\hline Outcome or subgroup title & No. of studies & $\begin{array}{l}\text { No. of partici- } \\
\text { pants }\end{array}$ & Statistical method & Effect size \\
\hline $\begin{array}{l}\text { 4.1 Skills (RCT/CRCT)-short-term } \\
\text { follow-up }\end{array}$ & 2 & 198 & $\begin{array}{l}\text { Std. Mean Difference (IV, Ran- } \\
\text { dom, 95\% Cl) }\end{array}$ & $0.21[-0.07,0.49]$ \\
\hline
\end{tabular}




\section{Analysis 4.1. Comparison 4: Skills, Outcome 1: Skills (RCT/CRCT)-short-term follow-up}

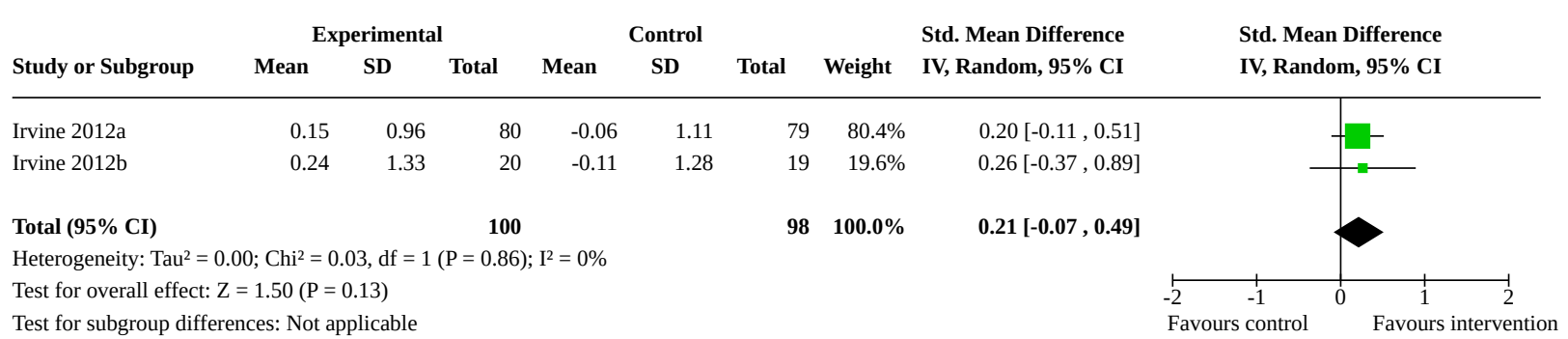

Comparison 5. Adverse personal outcomes

\begin{tabular}{lllll}
\hline Outcome or subgroup title & No. of studies & $\begin{array}{l}\text { No. of partici- } \\
\text { pants }\end{array}$ & Statistical method & Effect size \\
\hline $\begin{array}{l}\text { 5.1 Adverse personal (RCT/CRCT)- } \\
\text { short-term follow-up }\end{array}$ & 1 & 31 & $\begin{array}{l}\text { Std. Mean Difference (IV, Ran- } \\
\text { dom, 95\% Cl) }\end{array}$ & $-0.31[-1.02,0.40]$ \\
\hline 5.1.1 Short duration-face-to-face & 1 & 31 & $\begin{array}{l}\text { Std. Mean Difference (IV, Ran- } \\
\text { dom, 95\% Cl) }\end{array}$ & $-0.31[-1.02,0.40]$ \\
\hline
\end{tabular}

Analysis 5.1. Comparison 5: Adverse personal outcomes, Outcome 1: Adverse personal (RCT/CRCT)-short-term follow-up

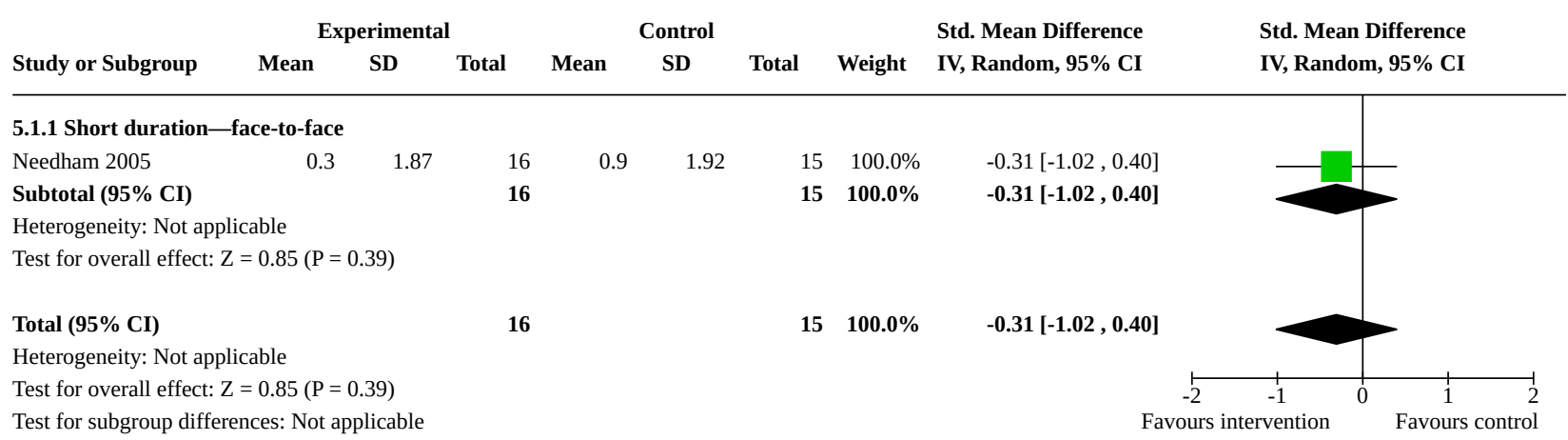

\section{ADDITIONAL TABLES}

Table 1. Characteristics of excluded studies

\begin{tabular}{ll}
\hline Study & Reason for exclusion \\
\hline Adams 2017 & Means not reported \\
\hline Beech 2003 & No control group \\
\hline Beech 2008 & No control group \\
\hline
\end{tabular}


Table 1. Characteristics of excluded studies (Continued)

\begin{tabular}{|c|c|}
\hline Cailhol 2007 & No control group \\
\hline Casalino 2015 & No control group \\
\hline Cooper 2006 & Study participants were not workers, but patients \\
\hline Fernandes 2002 & No control group \\
\hline Gates 2013 & Grant application for multi-program intervention. Results reported elsewhere \\
\hline Gerdtz 2012 & No control group \\
\hline Gertz 1980 & No control group \\
\hline Gillespie 2013 & No control group; multi-intervention program \\
\hline Gillespie 2014 & No control group; multi-intervention program \\
\hline Ishak 2002 & No pre-intervention or post-intervention measures reported \\
\hline Kang 2017 & Intervention aimed at preventing aggression between workers \\
\hline Kim 2018 & Did not evaluate en intervention \\
\hline Kowalenko 2014 & Conference abstract reporting on a multi-intervention program \\
\hline Li 2018 & Did not evaluate an intervention \\
\hline Lipscomb 2004a & No control group \\
\hline Lipscomb 2004b & Multi-intervention program reported with no specific findings related to education alone \\
\hline Lipscomb 2006 & Multi-intervention program reported with no specific findings related to education alone \\
\hline McElaney 2008 & Did not report on an intervention among healthcare workers \\
\hline Mclntosh 2003 & Outcomes not relevant \\
\hline Meehan 2006 & No control group \\
\hline Ore 2002 & No concurrent control group: control participants were selected after intervention \\
\hline Peek-Asa 2002 & Cross-sectional surveys before and after legislation enactment \\
\hline Rittenmeyer 2013 & Literature review \\
\hline Shah 1998 & No control group \\
\hline Vousden 1987 & Descriptive report-not an intervention study \\
\hline
\end{tabular}

Table 2. Intervention properties

\begin{tabular}{lllll}
\hline Study & Delivery & Length of intervention & Design & Follow-up
\end{tabular}


Table 2. Intervention properties (Continued)

\begin{tabular}{|c|c|c|c|c|c|}
\hline $\begin{array}{l}\text { Anderson } \\
2006\end{array}$ & Online & One session (3 hours) & CBA & short term & - aggression \\
\hline Arnetz 2000 & $\begin{array}{l}\text { Face-to-face } \\
\text { Reflective } \\
\text { practice }\end{array}$ & $\begin{array}{l}\text { Multiple sessions (as needed } \\
\text { over } 12 \text { months) }\end{array}$ & $\mathrm{CRCT}$ & long term & $\begin{array}{l}\text { - } \text { aggression } \\
\text { - knowledge }\end{array}$ \\
\hline Fitzwater 2002 & Face-to-face & Two sessions ( 2 hours each) & CRCT & short term & $\begin{array}{l}\text { - } \text { aggression } \\
\text { - confidence }\end{array}$ \\
\hline Irvine 2007 & Online & Self-paced & $\mathrm{RCT}$ & short term & $\begin{array}{l}\text { - } \text { knowledge } \\
\text { - } \text { self-efficacy } \\
\text { - attitudes }\end{array}$ \\
\hline Irvine 2012a & Online & Multiple sessions (2 weeks) & $\mathrm{RCT}$ & short term & $\begin{array}{l}\text { - } \text { knowledge } \\
\text { - } \text { self-efficacy } \\
\text { - } \text { attitudes } \\
\text { - } \text { skills }\end{array}$ \\
\hline Irvine 2012b & Online & Multiple sessions (2 weeks) & $\mathrm{CRCT}$ & short term & $\begin{array}{l}\text { - } \text { aggression } \\
\text { - } \text { self-efficacy } \\
\text { - } \text { attitudes, } \\
\text { - } \text { skills }\end{array}$ \\
\hline Ming 2019 & Face-to-face & One session (3 hours) & $\mathrm{RCT}$ & short term & $\begin{array}{l}\text { - } \text { self-efficacy } \\
\text { - attitudes }\end{array}$ \\
\hline $\begin{array}{l}\text { Needham } \\
2005\end{array}$ & Face-to-face & $\begin{array}{l}\text { Multiple sessions ( } 20 \text { times for } \\
50 \text { minutes over } 1 \text { week) }\end{array}$ & CRCT & short term & $\begin{array}{l}\text { - } \text { attitudes } \\
\text { - adverse events }\end{array}$ \\
\hline $\begin{array}{l}\text { Whittington } \\
1996\end{array}$ & Face-to-face & One session (7 hours) & CBA & short term & - aggression \\
\hline
\end{tabular}

CBA: controlled before and after study.

CRCT: cluster-randomized controlled study.

RCT: randomized controlled study.

\section{APPENDICES}

\section{Appendix 1. Search strategies}

\section{Campbell Collaboration Library of Systematic Reviews}

- aggress ${ }^{\star}$ OR violen* OR bully ${ }^{\star}$ OR harass* OR mob*

\section{Canadian Centre for Occupational Health and Safety}

- 'Violence'

- 'Violent'

- 'Aggression'

- 'Aggressive'

\section{ClinicalTrials.gov}

- education and training in "aggression OR violence"

Education and training for preventing and minimizing workplace aggression directed toward healthcare workers (Review) 
- aggression OR aggressive; intervention: education OR training

- violent OR violence: intervention: education OR training

- aggression OR aggressive OR violence OR violent OR bullying OR harassment OR mobbing | completed | interventional studies | education OR training

\section{World Health Organization International Clinical Trials Registry}

- "aggress* OR violen* OR bully* OR mobb* OR harass* OR Assault* OR disrupt OR abuse AND harass* OR assault* OR disrupt OR abuse"

\section{PubMed}

1. "Workplace Violence"[Mesh] OR "Violence/prevention and control"[Mesh] OR violence[tw] OR violent[tw] OR "Aggression"[Mesh] OR aggression*[tw] OR angry[tw] OR "Hostility"[Mesh] OR hostil*[tw] OR "inappropriate behavior"[tw] OR "Agonistic Behavior"[Mesh] OR "Bullying"[Mesh] OR bully*[tw] OR mob*[tw] OR harass* ${ }^{\star}[\mathrm{tw}]$ OR pester ${ }^{\star}[\mathrm{tw}]$ OR disrupt*[tw] OR incivility[tw] OR "emotional-verbal abuse"[tw] OR abus* [tw] OR assault*[tw]

2. work-related OR at work[tw] OR "Work"[Mesh] OR work[tw] OR worke* [tw] OR workplace*[tw] OR work place*[tw] OR work site* [tw] OR occupation*[tw] OR "Occupations"[MeSH] OR "Occupational Groups"[MeSH] OR job*[tw] OR "Occupational Health"[MeSH] OR "occupational health"

3. \#1 AND \#2

4. "Health Personnel"[Mesh] OR "Personnel, Hospital"[Mesh] OR "health care worker"[tw] OR "health care workers"[tw] OR "health care personnel"[tw] OR "health personnel"[tw] OR "health-personnel"[tw] OR "health provider"[tw] OR "health providers"[tw] OR "health care provider"[tw] OR "health care providers"[tw] OR "health staff"[tw] OR "health care staff"[tw] OR "healthcare staff"[tw] OR "health professional"[tw] OR "health care professional"[tw] OR "healthcare professional"[tw] OR "health worker"[tw] OR "medical staff"[tw] OR "medical personnel"[tw] OR "medical professional"[tw] OR "medical worker"[tw] OR "medical workers"[tw] OR "medical provider"[tw] OR "military-medical personnel" [tw] OR "Physicians"[Mesh] OR "physician"[tw] OR "physicians"[tw] OR "doctor"[tw] OR "practitioner"[tw] OR "clinician"[tw] OR "nursing staff"[tw] OR "Nurses"[Mesh] OR "nurse"[tw] OR "nurses"[tw] OR "nursing assistant"[tw] OR "nursing assistants"[tw] OR "Nurses' Aides"[Mesh] OR "Nurse Midwives"[Mesh] OR "midwife"[tw] OR "midwives"[tw] OR "dental personnel"[tw] OR "dental staff"[tw] OR "Dentists"[Mesh] OR "dentist"[tw] OR "dentists"[tw] OR "dental assistant"[tw] OR "dental assistants"[tw] OR "Dental Assistants"[Mesh] OR "Pharmacists"[Mesh] OR "pharmacist"[tw] OR "Physical Therapists"[Mesh] OR "physical therapist"[tw] OR "physical therapists"[tw] OR "physiotherapist"[tw] OR "physiotherapists"[tw] OR "therapist"[tw] OR "therapists"[tw] OR "Physical Therapist Assistants"[Mesh] OR "technician"[tw] OR "technicians"[tw] OR "radiographer"[tw] OR "radiographers"[tw] OR "emergency medical services"[tw] OR "Emergency Medical Services"[MeSH] OR "transporting patients"[tw] OR "patient transport"[tw] OR "Ambulances"[Mesh] OR "Allied Health Personnel"[Mesh] OR "paramedic"[tw] OR "paramedics"[tw] OR "paramedical personnel"[tw] OR "health manager"[tw] OR "health care manager"[tw] OR "healthcare manager"[tw] OR "clinical officer"[tw] OR "reception"[tw]

5. \#3 AND \#4

6. "Health Personnel/education"[Mesh] OR "Nursing Staff, Hospital/education"[Mesh] OR "Health Occupations/education"[Mesh] OR education[tw] OR "Inservice Training"[Mesh] OR training[tw] OR inservice[tw] OR in-service[tw] OR "Staff Development"[Mesh] OR program* OR "aggression management" (372143)

7. \#5 AND \#6

8. ("Comparative Study" [Publication Type] OR effectiveness OR program OR intervention OR reduction OR effect*[ti] OR evaluation OR decrease* OR "prevention and control" OR measures OR improve*[tiab])

9. \#7 AND \#8

10.\#9 NOT ("Child Abuse"[Mesh])

11. (randomized controlled trial [pt] OR controlled clinical trial [pt] OR randomized [tiab] OR randomised [tiab] OR placebo [tiab] OR clinical trials as topic [mesh: noexp] OR randomly [tiab] OR trial [ti] NOT (animals [mh] NOT humans [mh]))

12.\#10 AND \#11

13."Controlled Before-After Studies"[Mesh] OR "controlled before-after study"[tw] OR "controlled before-after studies"[tw] OR "CBA study" OR "CBA studies" OR "before-after study"[tw] OR "before-after studies"[tw] OR "Prospective Studies"[Mesh] OR prospective study OR "longitudinal studies"[MeSH]

14.\#10 AND \#13

\section{Embase}

1. 'workplace violence'/de OR 'violence'/de OR 'violence' OR 'violent' OR 'aggression'/de OR aggression* OR 'angry' OR 'hostility'/de OR hostil* OR 'inappropriate behavior' OR 'agonistic behavior'/de OR 'bullying'/de OR bully* OR mob* OR harass* OR pester* OR disrupt* OR incivility OR 'emotional-verbal abuse' OR abus* OR assault*

2. 'work related' OR 'at work' OR 'work'/de OR 'work' OR worke^ OR workplace* OR work NEAR/1 place* OR work NEAR/1 site* OR occupation* OR 'occupations'/de OR 'occupational groups'/de OR job* OR 'occupational health'/de OR 'occupational health'

3. \#1 AND \#2

Education and training for preventing and minimizing workplace aggression directed toward healthcare workers (Review) 
4. 'health care personnel'/de OR 'hospital personnel'/de OR 'hospital personnel' OR 'health care worker' OR 'health care workers' OR 'health care personnel' OR 'health personnel' OR 'health-personnel' OR 'health provider' OR 'health providers' OR 'health care provider' OR 'health care providers' OR 'medical staff' OR 'medical personnel' OR 'medical professional' OR 'medical worker' OR 'medical workers' OR 'dental personnel' OR 'dental staff' OR 'dentist' OR 'dentists' OR 'dental assistant' OR 'dental assistants' OR 'nursing staff' OR 'nurses'/ de OR 'nurse' OR 'nurses' OR 'nursing assistants' OR 'nursing assistant'/de OR 'nursing assistant' OR 'nurse midwife'/de OR 'nurse midwife' OR 'midwife' OR 'midwives' OR 'military-medical personnel' OR 'physician'/de OR 'physician' OR 'physicians' OR 'emergency medical services' OR 'transporting patients' OR 'patient transport' OR 'ambulance'/de OR 'ambulance' OR 'paramedical personnel'/de OR 'paramedical personnel' OR paramedic OR paramedics OR 'health manager' OR 'health care manager' OR 'healthcare manager' OR 'clinical officer' OR 'reception'

5. \#3 AND \#4

6. 'inservice training'/de OR 'in service training' OR 'in service' OR 'inservice' OR 'training' OR 'allied health education' OR 'staff development'/de OR 'personnel management'/de OR aggression NEAR/1 management OR program* OR 'health personnel' NEAR/5 education OR nurse NEAR/5 education OR 'medical profession' NEAR/5 education OR 'interprofessional education'

7. \#5 AND \#6

8. 'comparative study':it OR 'comparative study'/exp OR 'intermethod comparison'/exp OR effectiveness OR program OR intervention OR reduction OR effect*:ti OR evaluation OR decrease* OR 'prevention and control'/de OR measures OR improve*:ab,ti

9. \#7 AND \#8

10.\#9 NOT 'child abuse'/de

11.\#10 AND [embase]/lim

12.\#11 NOT [medline]/lim

13.'randomized controlled trial'/exp OR 'controlled study'/exp OR 'randomization'/exp OR 'double blind procedure'/exp OR 'single blind procedure'/exp OR 'clinical trial'/exp OR 'crossover procedure'/exp OR 'follow up'/exp OR 'prospective study'/exp OR (singl* OR doubl* OR trebl* OR tripl*) NEXT/5 (mask* OR blind ${ }^{\star}$ OR method ${ }^{\star}$ ) OR clinical NEXT/3 trial ${ }^{\star}$ OR 'placebo'/exp OR placebo*:ab,ti OR random*:ab,ti OR latin NEXT/3 square OR 'comparative study'/exp OR ((control* OR prospective* OR volunteer ${ }^{\star}$ ) NEAR/3 (trial* OR method $^{\star}$ OR stud $\left.^{\star}\right)$ ):ab,ti OR crossover* OR cross NEXT/1 over* NOT ('nonhuman'/exp NOT 'human'/exp)

14.\#12 AND \#13

15.\#14 AND 'clinical trial'/de

\section{PsycInfo}

1. workplace violence/ OR violen*.sh. OR aggressive behavior/ OR aggression.sh. OR anger.sh. OR hostility.ti. OR hostility.ab. OR inappropriate behavio*.ti. OR inappropriate behavio*.ab. OR agnostic behavior*.af. OR bullying/ OR bully.sh. OR mob.sh. OR harass*.ti.

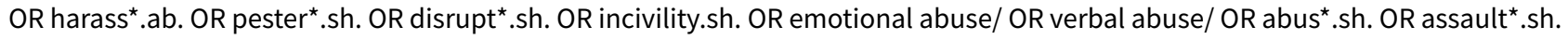

2. Work-related.sh. OR work.sh. OR worke ${ }^{\star}$.sh. OR work site ${ }^{\star}$.af. OR occupation*.sh. OR occupations/OR job*..sh. OR occupational health.sh. OR occupational health.af.

3. \#1 AND \#2

4. health personnel/ OR allied health personnel/ OR medical personnel/ OR mental health personnel/ OR counsellors/ OR counselor ${ }^{*}$.sh. OR home care personnel/ OR social workers/ OR nurses/OR nurses.sh. OR dentists/OR therapists.sh. OR optometrists/OR pharmacists/ OR physicians/ OR physicians.sh. OR general practitioners/ OR gynaecologists/ OR internists/ OR neurologists/ OR obstetricians/ OR pathologists/ OR paediatricians/ OR surgeons/OR psychiatric hospital staff/ OR psychiatric aides/ OR psychiatrists/ OR attendants/ OR clinicians/

5. \#3 AND \#4

6. inservice training/ OR personnel training/ OR on the job training/ OR professional development/ OR education.sh. OR training.sh. OR inservice.sh. OR in-service.af. OR staff development.af. OR health personnel education.af. OR training program.af. OR aggression management.af. OR staff education.af.

7. \#5 AND \#6

8. comparative study.ti. OR comparative study.ab. OR effectiveness.af. OR program.af. OR intervention.af. OR reduction.af. OR effect.af. OR evaluation.af. OR decrease ${ }^{\star}$.af. OR prevention and control.af. OR measures.af. OR improve*.ti. OR improve*.ab

9. \#7 AND \#8

10.treatment outcome.af. OR clinical trial.af. OR randomized.ti. OR randomized.ab. OR placebo.ti. OR placebo.ab. OR clinical trials/ OR randomly.ti. OR randomly.ab. OR trial.ti. NOT animals/ NOT animals.sh.

11.\#9 AND \#10

12.longitudinal study.af. OR controlled before-after stud*.af. OR CBA stud*.af. OR before-after stud*.af. OR prospective studies/ OR prospective study.md. OR prospective stud*.af.

13.\#9 AND \#12

\section{CINAHL}


1. (MH "Workplace Violence") OR (MH "Violence/PC") OR (MW violence) OR (MW violent) OR (MH "Aggression") OR (MW aggression*) OR (MW angry) OR (TI hostility) OR (AB hostility) OR (TI "inappropriate behavior") OR (AB "inappropriate behavior") OR (TX "agnostic behavior") OR (MH Bullying) OR (MW bully) OR (MW mob*) OR (MW harass*) OR (MW pester ${ }^{\star}$ ) OR (MW disrupt*) OR (MW incivility) OR (MW "emotionalverbal abuse") OR (MW abus*) OR (MW assault*)

2. (MW work-related OR "at work") OR (MH work) OR (MW work) OR (MW worke*) OR (MW workplac*) OR (MW "work site*") OR (MW occupation ${ }^{\star}$ OR (MH "occupations and professions") OR (MH "named groups by occupation") OR (MW job*) OR (MH "occupational health") OR (TX "occupational health")

3. \#1 AND \#2

4. (MH "health personnel") OR (MH "personnel, health facility") OR (MW "health care worker") OR (MW "health care workers") OR (MW "health care personnel") OR (MW "health personnel") OR (MW "health-personnel") OR (MW "health provider") OR (MW "health providers") OR (MW "health care provider") OR (MW "health care providers") OR (MW "health staff") OR (MW "health care staff") OR(MW "healthcare staff") OR (MW "health professional") OR (MW "health care professional") OR (MW "healthcare professional") OR (MW "health worker") OR (MW "medical staff") OR (MW "medical personnel") OR (MW "medical professional") OR (MW "medical worker") OR (MW "medical workers") OR (MW "medical provider") OR (MW "military-medical personnel") OR (MH "physicians") OR (MW "physician") OR (MW "physicians") OR (MW "doctor") OR (MW "practitioner") OR (MW "clinician") OR (MW "nursing staff") OR (MH "nurses") OR (MW "nurse") OR (MW "nurses") OR (MW "nursing assistant") OR (MW "nursing assistants") OR (MH "nursing assistants") OR (MH "nurse midwives") OR (MW "midwife") OR (MW "midwives") OR (MW "dental personnel") OR (MW "dental staff") OR (MH "dentists") OR (MW "dentist") OR (MW "dentists") OR (MW "dental assistant") OR (MW "dental assistants") OR (MH "dental assistants") OR (MH "pharmacists") OR (MW "pharmacist") OR (MH "physical therapists") OR (MW "physical therapist") OR (MW "physical therapists") OR (MW "physiotherapist") OR (MW "physiotherapists") OR (MW "therapist") OR (MW "therapists") OR (MH "physical therapist assistants") OR (MW "technician") OR (MW "technicians") OR (MW "radiographer") OR (MW "radiographers") OR (MW "emergency medical services") OR (MH "emergency medical services") OR (MW "transporting patients") OR (MW "patient transport") OR (MH "ambulances") OR (MH "allied health personnel") OR (MW "paramedic") OR (MW "paramedics") OR (MW "paramedical personnel") OR (MW "health manager") OR (MW "health care manager") OR (MW "healthcare manager") OR (MW "clinical officer") OR (MW "reception")

5. \#3 AND \#4

6. (MH "Health Personnel/ED") OR (MH "Nursing Staff, Hospital/ED") OR (MH "Health Occupations/ED") OR (MW education) OR (TX "inservice training") OR (MW training) OR (MW inservice) OR (MW in-service) OR (MH "Staff Development") OR program* OR (TX "aggression management")

7. \#5 AND \#6

8. ( $\mathrm{TI}$ "comparative study") OR (AB "comparative study") OR effectiveness OR program OR (TX intervention) OR (TX reduction) OR (TI effect ${ }^{\star}$ ) OR (TX evaluation) OR (TX decrease ${ }^{\star}$ ) OR (TX "prevention and control") OR (TX measures) OR (TI improve*) OR (AB improve*)

9. \#7 AND \#8

10.(PT “randomized controlled trial”) OR (PT clinical trial) OR (TI randomized) OR (AB randomized) OR (TI placebo) OR (AB placebo) OR (MM "clinical trials") OR (TI randomly) OR (AB randomly) OR (TI trial) NOT (MH animals NOT MH human)

11.\#9 AND \#10

12.(MH "Controlled Before-After Studies") OR (TX "controlled before-after study") OR (TX "controlled before-after studies") OR (TX "CBA study") OR (TX "CBA studies") OR (TX "before-after study") OR (TX "before-after studies") OR (MH "Prospective Studies") OR (TX "prospective study")

13.\#9 AND \#12

Appendix 2. Data extraction form

Categories Subcategories

Publication details Study authors and email address of corresponding author

Date of publication

Title

Journal name, volume, issue, and pages

Methods

Study design (e.g. RCT/cluster RCT/CBA) including sampling, group allocation, and treatment of missing data

Study location/s

Study setting/s 
(Continued)

Withdrawals

Participants

Health worker type/s

Total number, number of health worker type subpopulations and proportions (\%)

Mean age or age range

Gender

Workplace/s (e.g. mental health, emergency department)

Work setting/s (e.g. hospital inpatient, hospital outpatient, community)

Work sector/s (e.g. public, private, non-government)

Inclusion and exclusion criteria

Intervention/s

Description of intervention and co-interventions (especially noting whether bundled with other organizational interventions)

Targeted knowledge, attitudes, and skills

Comparison

Content of both intervention and control conditions, and co-interventions

Duration

Intensity (e.g. frequency or levels of intervention)

Number commencing

Number completing

Adherence to protocol

Description of primary and secondary outcomes specified and collected
Measurement instruments used and validation status (e.g. reported/not reported)
Time points reported
Controlling for biasing or confounding effects of co-interventions

Notes $\quad$ Funding for study

Notable conflicts of interest of study authors

\section{Appendix 3. Calculation of total number of participants in cluster RCTs}

\begin{tabular}{lllll}
\hline & $\begin{array}{l}\text { Intervention group-be- } \\
\text { fore correction }\end{array}$ & $\begin{array}{l}\text { Intervention } \\
\text { group-after correc- } \\
\text { tion }\end{array}$ & $\begin{array}{l}\text { Control group-be- } \\
\text { fore correction }\end{array}$ & $\begin{array}{l}\text { Control group-af- } \\
\text { ter correction }\end{array}$ \\
\hline Arnetz 2000 & 353 & 149 & 333 & 142 \\
\hline
\end{tabular}




\section{(Continued)}

\begin{tabular}{lcccc} 
Fitzwater 2002 & 10 & 5 & 10 & 5 \\
\hline Irvine 2012b & 58 & 20 & 45 & 19 \\
\hline Needham 2005 & 30 & 16 & 28 & 15 \\
\hline
\end{tabular}




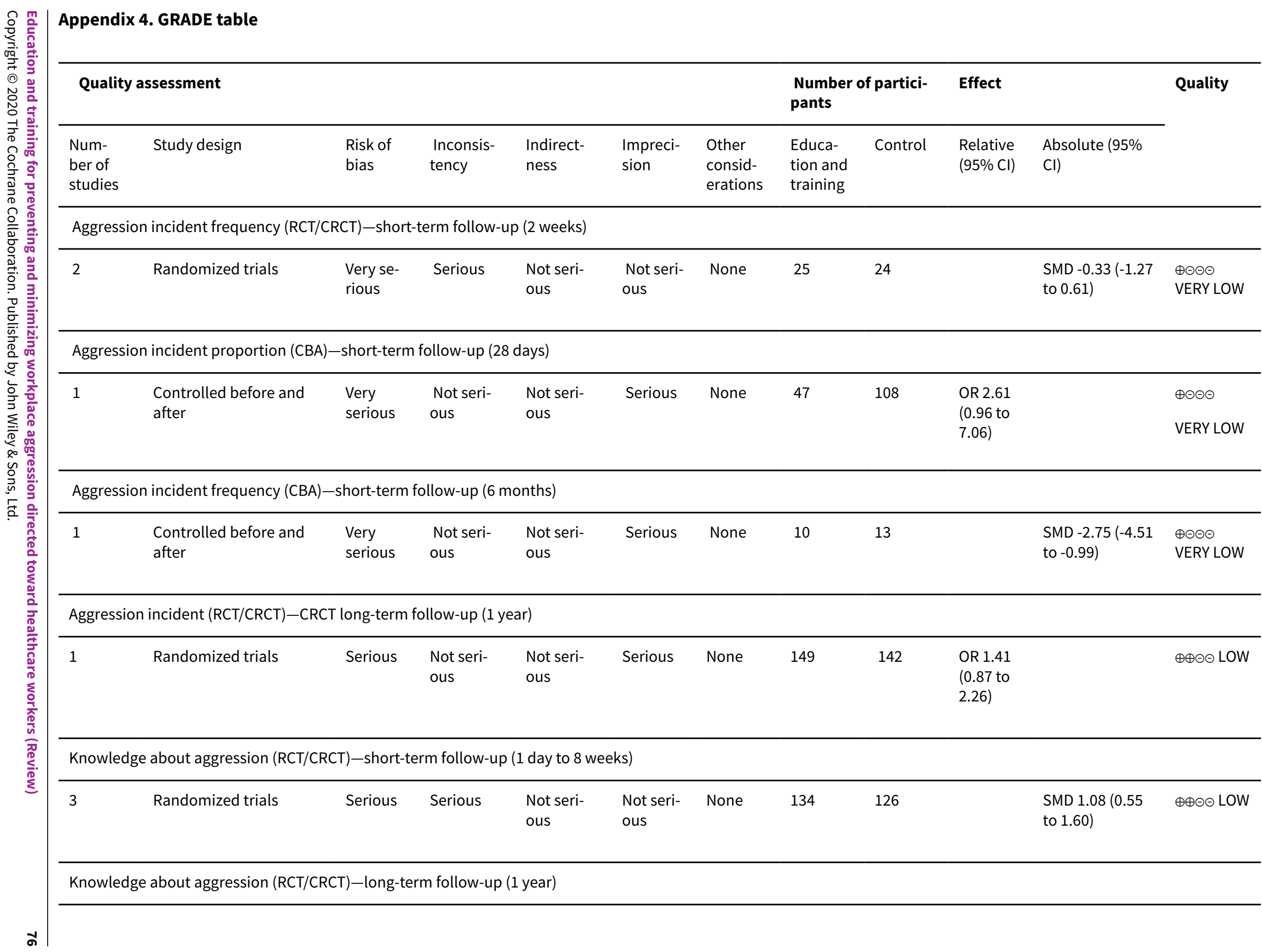




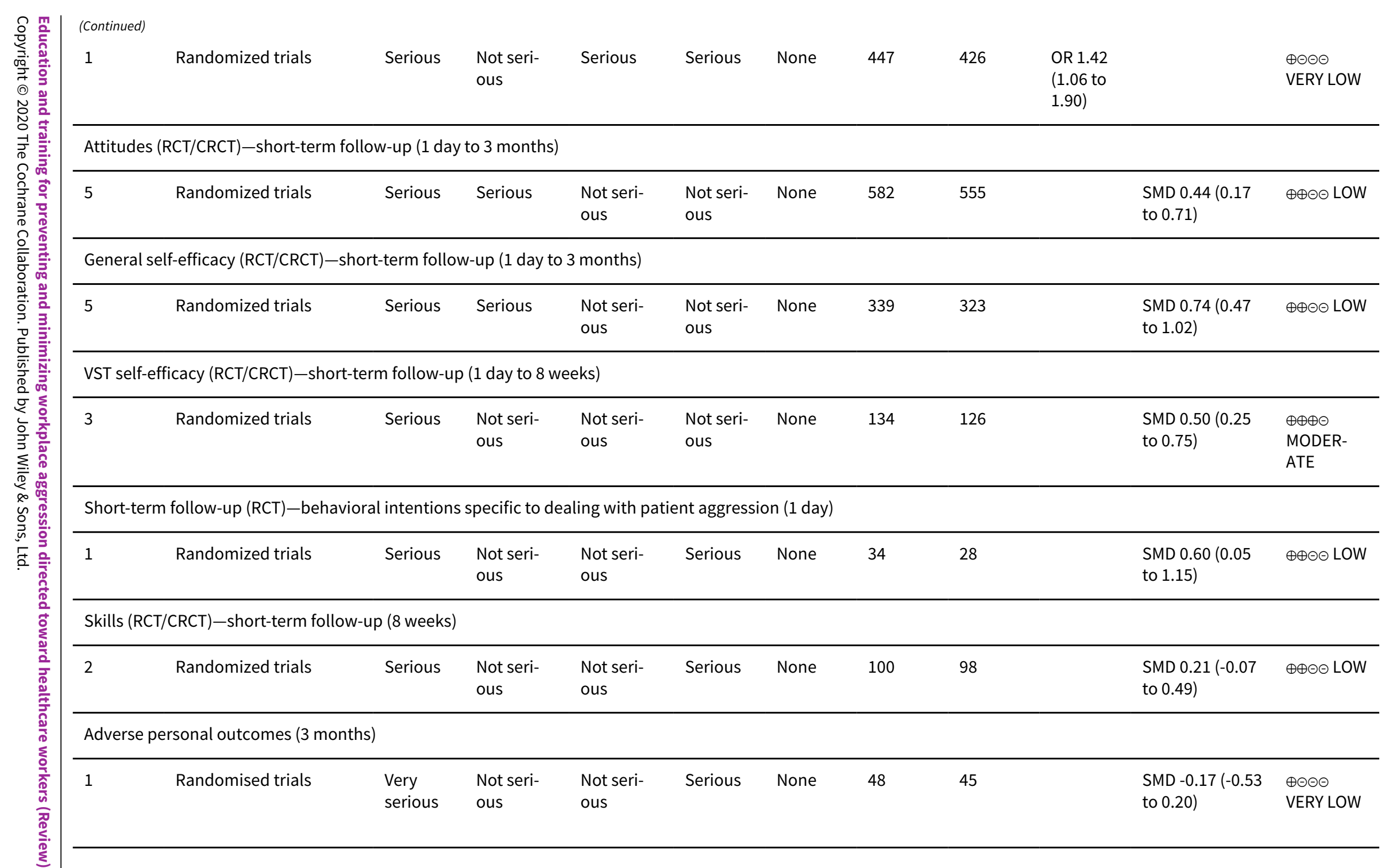


CBA: controlled before and after study.

Cl: confidence interval.

CRCT: cluster-randomized controlled study.

OR: odds ratio.

$\mathrm{RCT}$ : randomized controlled trial.

SMD: standardized mean difference.

\section{H IST O RY}

Protocol first published: Issue 9, 2015

Review first published: Issue 9, 2020

\section{CONTRIBUTIONS OF AUTHORS}

Co-ordinating the review: SGe, DH.

Designing the protocol: DH, HR, JP, AH, TD, SR.

Designing search strategies: DH, TD, in collaboration with Kaisa Neuvonen, formerly Trials Search Co-ordinator of the Cochrane Occupational Safety and Health Group.

Writing the protocol: DH, HR, JP, AH, TD, SR, SG, BM-J

Providing general advice on the protocol: DH, HR, JP, AH, TD, SR, SG, BM-J.

Undertaking the meta-analyses: SGe, TD, DH

Writing the review: SGe, DH, HR, TD, JP, AH, SR, SG, BM-J

\section{DECLARATIONS OF INTEREST}

Steve Geoffrion: none known.

Danny Hills: none known.

Heather Ross: none known.

Therese Dalsbø: none known.

Jacqueline Pich: none known.

April Hill: none known.

Sanaz Riahi: none known.

Begoña Martínez-Jarreta: none known.

Stephane Guay: none known.

\section{SOURCES OF SUPPORT}

\section{Internal sources}

- New source of support, Canada

A career grant awarded to the first author by the Fonds de recherche du Québec-Institut Robert-Sauvé en Santé et Sécurité au Travail

\section{External sources}

- No external source of support, Canada

No external source of support 


\section{DIFFERENCES BETWEEN PROTOCOLAND REVIEW}

- Steve Geoffrion was added to the team to compute the analyses and to co-ordinate writing and submission of the review. He also updated the search for new publications since the time the protocol was published

- Authorship was modified given the implications of all authors. Steve Geoffrion became first author as he led analyses and writing and submission of the review. Stéphane Guay became last author as he acted as the senior author and has authored the most publications on workplace aggression

\section{N DEX TERMS}

\section{Medical Subject Headings (MeSH)}

*Aggression; Bias; Controlled Before-After Studies; Exposure to Violence [prevention \& control]; Health Personnel [*education]; Nursing Assistants [education]; Nursing Staff [education]; Randomized Controlled Trials as Topic; Workplace Violence [* prevention \& control]

\section{MeSH check words}

Humans 